\title{
SPACE VEHICLE TESTING
}

\author{
A Master's Project \\ presented to \\ the Faculty of California Polytechnic State University, \\ San Luis Obispo
}

\author{
In Partial Fulfillment \\ of the Requirements for the Degree \\ Master of Science in Aerospace Engineering
}

by

Charlotte Ann Belsick

December 2012 
(C) 2012

Charlotte Ann Belsick

ALL RIGHTS RESERVED 


\section{COMMITTEE MEMBERSHIP}

TITLE:

AUTHOR:

DATE SUBMITTED:

COMMITTEE CHAIR:

COMMITTEE MEMBER:

COMMITTEE MEMBER:

COMMITTEE MEMBER:
Space Vehicle Testing

Charlotte Ann Belsick

December 2012
Dr. Jordi Puig-Suari, Professor Aerospace Engineering

Bruce Arnheim, The Aerospace Corporation, Systems Director

Ron Moore, Lockheed Martin Space Systems Company, Systems Engineering Manager

Dr. Eric Mehiel, Department Chair, Aerospace Engineering 


\title{
ABSTRACT \\ Space Vehicle Testing \\ Charlotte Ann Belsick
}

\begin{abstract}
Requirement verification and validation is a critical component of building and delivering space vehicles with testing as the preferred method. This Master's Project presents the space vehicle test process from planning through test design and execution. It starts with an overview of the requirements, validation, and verification. The four different verification methods are explained including examples as to what can go wrong if the verification is done incorrectly. Since the focus of this project is on test, test verification is emphasized. The philosophy behind testing, including the "why" and the methods, is presented. The different levels of testing, the test objectives, and the typical tests are discussed in detail. Descriptions of the different types of tests are provided including configurations and test challenges. While most individuals focus on hardware only, software is an integral part of any space product. As such, software testing, including mistakes and examples, is also presented. Since testing is often not performed flawlessly the first time, sections on anomalies, including determining root cause, corrective action, and retest is included. A brief discussion of defect detection in test is presented.
\end{abstract}

The project is actually presented in total in the Appendix as a Power Point document.

Keywords: test, verification, software, hardware, anomaly 


\section{ACKNOWLEDGMENTS}

I'd like to thank Bruce Arnheim, The Aerospace Corporation, for his counsel and guidance and Ron Moore, Lockheed Martin Space Systems Company, for his advice in the development of this project. I would also like to thank Michael Belsick, Lockheed Martin Space Systems Company for his relentless reminders, reviews, and support without which this project would still be work in process. 


\section{TABLE OF CONTENTS}

Appendix A Space Vehicle Testing Power Point Document................................. 1

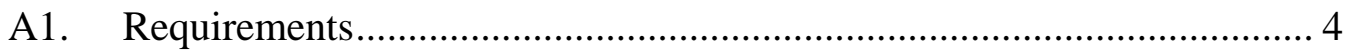

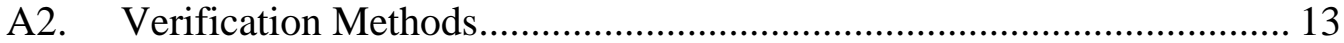

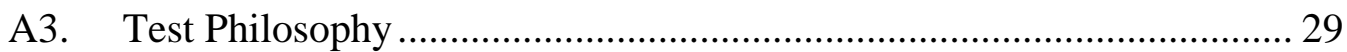

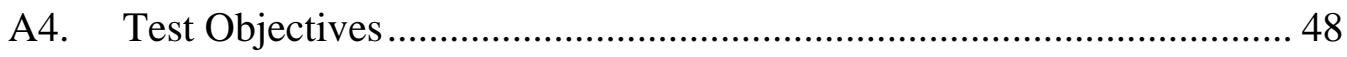

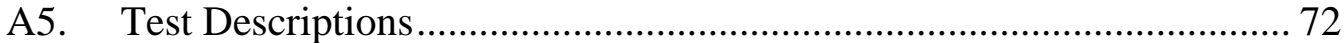

A6. Software Testing ...................................................................... 112

A7. Anomalies.............................................................................. 142

A8. Defect Detection in Test...................................................................... 159

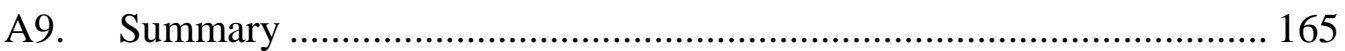

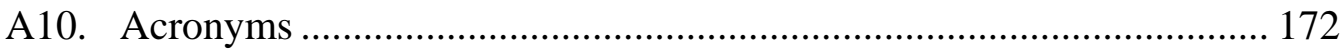

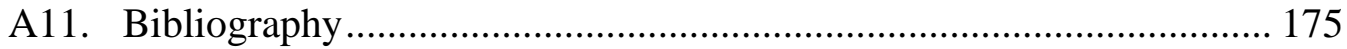


A1. Requirements

A2. Verification Methods

A3. Test Philosophy

A4. Test Objectives

A5. Test Descriptions

A6. Software Testing

A7. Anomalies

A8. Defect Detection in Test

A9. Summary

A10. Acronyms

A11. Bibliography 


\section{Space Vehicle Testing}

Charlotte Belsick

AERO 599

Master's Project

Fall Quarter 2012 


\section{Agenda}

- Requirements

- Verification Methods

- Test Philosophy

- Test Objectives

- Test Descriptions

- Software Testing

- Anomalies

- Defect Detection in Test

- Summary 


\section{REQUIREMENTS}




\section{Where Do Requirements Come From?}

- Established to assure that product performs as needed in the operational environments

- Product performance

- Margins

- Proof of product development

- Proof of product build

- Standard testing requirements are levied on the aerospace community

- Function of type of product

- Function of level of integration

- Establishes environments

- Provides preferred sequence

- Product specifications generated

- Product performance with margin

- Environments

- Levied requirements 


\section{Typical Environment Requirements}

\begin{tabular}{|c|c|c|c|c|c|c|c|c|c|c|c|}
\hline Test Units & $\begin{array}{l}\text { Electrical } \\
\text { and } \\
\text { Electronic }\end{array}$ & Antemia & MMA & $\begin{array}{l}\text { Solar } \\
\text { Array }\end{array}$ & Battery & $\begin{array}{l}\text { Valve or } \\
\text { Propulsion } \\
\text { Component }\end{array}$ & $\begin{array}{l}\text { Pressure } \\
\text { Vessel or } \\
\text { Component }\end{array}$ & Thruster & Themal & Optical & $\begin{array}{l}\text { Structural } \\
\text { Components }\end{array}$ \\
\hline Inspection & $\mathrm{K}$ & $\mathrm{K}$ & $\mathrm{K}$ & $\mathrm{K}$ & $\mathrm{K}$ & $\mathrm{K}$ & $\mathrm{K}$ & $\mathrm{K}$ & $\mathrm{K}$ & $\mathrm{K}$ & $\mathrm{K}$ \\
\hline Specification & $\mathrm{R}$ & $\mathrm{R}$ & $\mathrm{R}$ & $\mathrm{R}$ & $\mathrm{R}$ & $\mathrm{R}$ & $\mathrm{R}$ & $\mathrm{B}$ & & \begin{tabular}{|l|}
$\mathrm{B}$ \\
\end{tabular} & $\mathrm{ER}$ \\
\hline Leakage & ER & - & $\mathrm{R}$ & - & $\mathrm{R}$ & $\mathrm{R}$ & $\mathrm{R}$ & \multicolumn{4}{|c|}{$\begin{array}{l}\text { All units fall into one or more of } \\
\text { these categories }\end{array}$} \\
\hline Shock & $\mathrm{R}$ & ER & ER & ER & $\mathrm{R}$ & ER & ER & ER & ER & ER & ER \\
\hline $\begin{array}{l}\text { Vibration } \\
\text { or } \\
\text { Acoustic }\end{array}$ & $\mathrm{R}$ & $\mathrm{R}$ & $\mathrm{R}$ & $\mathrm{R}$ & $\mathrm{R}$ & $\mathrm{R}$ & $\mathrm{R}$ & $\mathrm{R}$ & $\mathrm{R}$ & $\mathrm{R}$ & ER \\
\hline Acceleration & ER & ER & ER & ER & ER & \multicolumn{3}{|c|}{${ }^{"} R "=$ Required Test } & - & & ER \\
\hline Thermal Cycle & $\mathrm{R}$ & ER & ER & ER & $\mathrm{R}$ & का & Lा & DT & ER & ER & ER \\
\hline Thermal Vacumim & $\mathrm{R}$ & $\mathrm{R}$ & $\mathrm{R}$ & $\mathrm{R}$ & $\mathrm{R}$ & $\mathrm{R}$ & $\mathrm{R}$ & $\mathrm{R}$ & $\mathrm{R}$ & 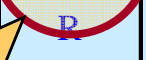 & - \\
\hline Olimatic & $\mathrm{ER}$ & \multirow{3}{*}{\multicolumn{3}{|c|}{$\begin{array}{l}\text { Ensemble of } \\
\text { environmental tests }\end{array}$}} & תחב & ER & ER & ER & & ER & ER \\
\hline Pressure & \multirow{2}{*}{$\begin{array}{c}\mathrm{E} \\
\mathrm{r}\end{array}$} & & & & & $\mathrm{R}$ & $\mathrm{R}$ & \multirow{2}{*}{\multicolumn{4}{|c|}{$\begin{array}{l}\text { "ER" = Evaluation required } \\
\text { with technical rationale }\end{array}$}} \\
\hline EMC & & & & & & ER & ER & & & & \\
\hline Life & ER & ER & $\mathrm{R}$ & ER & $\mathrm{R}$ & $\mathrm{R}$ & ER & $\bar{R}$ & ER & ER & ER \\
\hline Burst Pressure & - & - & ER & - & $\mathrm{R}$ & & $\mathrm{R}$ & $\mathrm{R}$ & ER & - & - \\
\hline Static Load & ER & ER & ER & ER & $\mathrm{R}$ & - & ER & - & - & - & $\mathrm{R}$ \\
\hline
\end{tabular}

Ref: TR-2004(8583)-1 Rev A (MIL-STD 1540E) 


\section{More Information is Available}

- For each test the matrix points to the sections in MIL-STD-1540 for detailed requirements

- Associated standards referenced for additional information and application

\begin{tabular}{|c|c|c|c|c|c|c|c|c|c|c|c|}
\hline Test Units & $\begin{array}{c}\text { Electrical } \\
\quad \text { and } \\
\text { Electronic } \\
\end{array}$ & Antenna & MMA & $\begin{array}{l}\text { Solar } \\
\text { Array } \\
\end{array}$ & Battery & $\begin{array}{l}\text { Valve or } \\
\text { Propulsioit } \\
\text { Component } \\
\end{array}$ & $\begin{array}{l}\text { Presstire } \\
\text { Vessel or } \\
\text { Component } \\
\end{array}$ & 2 hruster & Thermal & Optical & $\begin{array}{c}\text { Structural } \\
\text { Components } \\
\end{array}$ \\
\hline Inspection & $\mathrm{R}$ & $\mathrm{R}$ & $\mathrm{R}$ & $\mathrm{R}$ & $\mathrm{R}$ & $\mathrm{R}$ & $\mathrm{R}$ & $\mathrm{R}$ & $\mathrm{R}$ & $\mathrm{R}$ & $\mathrm{R}$ \\
\hline $\begin{array}{l}\text { Specification } \\
\text { Performance }\end{array}$ & $\mathrm{R}$ & $\mathrm{R}$ & $\mathrm{R}$ & $\mathrm{R}$ & $\mathrm{R}$ & $\mathrm{R}$ & $\mathrm{R}$ & $\mathrm{R}$ & $\mathrm{R}$ & $\mathrm{R}$ & ER \\
\hline Leakage & \multicolumn{11}{|c|}{ Example: Associated requirements references for pressure testing (Section 2) } \\
\hline $\begin{array}{l}\text { Shock } \\
\begin{array}{l}\text { Vibration } \\
\text { or }\end{array}\end{array}$ & \multicolumn{3}{|c|}{ AIAA S-080-1998 } & \multicolumn{8}{|c|}{$\begin{array}{l}\text { Space Systems-Metallic Pressure Vessels, Pressurized Structures } \\
\text { and Pressure Components }\end{array}$} \\
\hline Acoustic & \multicolumn{3}{|c|}{ AIAA S-081-2000 } & \multirow{2}{*}{\multicolumn{8}{|c|}{ Space Systems-Composite Overwrapped Pressure Vessels }} \\
\hline Acceleration & & & & & & & & & & & \\
\hline Thermal Cycle & & ER & ER & $\overline{E R}$ & $\mathrm{R}$ & ER & ER & ER & ER & ER & ER \\
\hline Thermal Vacuurs & $\mathrm{R}$ & $\mathrm{R}$ & $\mathrm{R}$ & $\mathrm{R}$ & $\mathrm{R}$ & $\mathrm{R}$ & $\mathrm{R}$ & $\mathrm{R}$ & $\mathrm{R}$ & $\mathrm{R}$ & - \\
\hline Climatic & ER & ER & ER & ER & ER & ER & ER & ER & ER & ER & ER \\
\hline Pressirte & ER & - & ER & - & $\mathrm{R}$ & $\mathrm{R}$ & $\mathrm{R}$ & ER & ER & - & - \\
\hline EMC & $\mathrm{R}$ & $\mathrm{R}$ & ER & ER & ER & ER & ER & ER & ER & ER & ER \\
\hline Life & ER & ER & $\mathrm{R}$ & ER & $\mathrm{R}$ & $\mathrm{R}$ & ER & $\mathrm{R}$ & ER & ER & ER \\
\hline Burst Pressure & - & - & ER & - & $\mathrm{R}$ & $\mathrm{R}$ & $\mathrm{R}$ & $\mathrm{R}$ & ER & - & - \\
\hline Static Load & ER & ER & ER & ER & $\mathrm{R}$ & - & ER & - & - & - & $\mathrm{R}$ \\
\hline
\end{tabular}




\section{"VEE" Diagram}

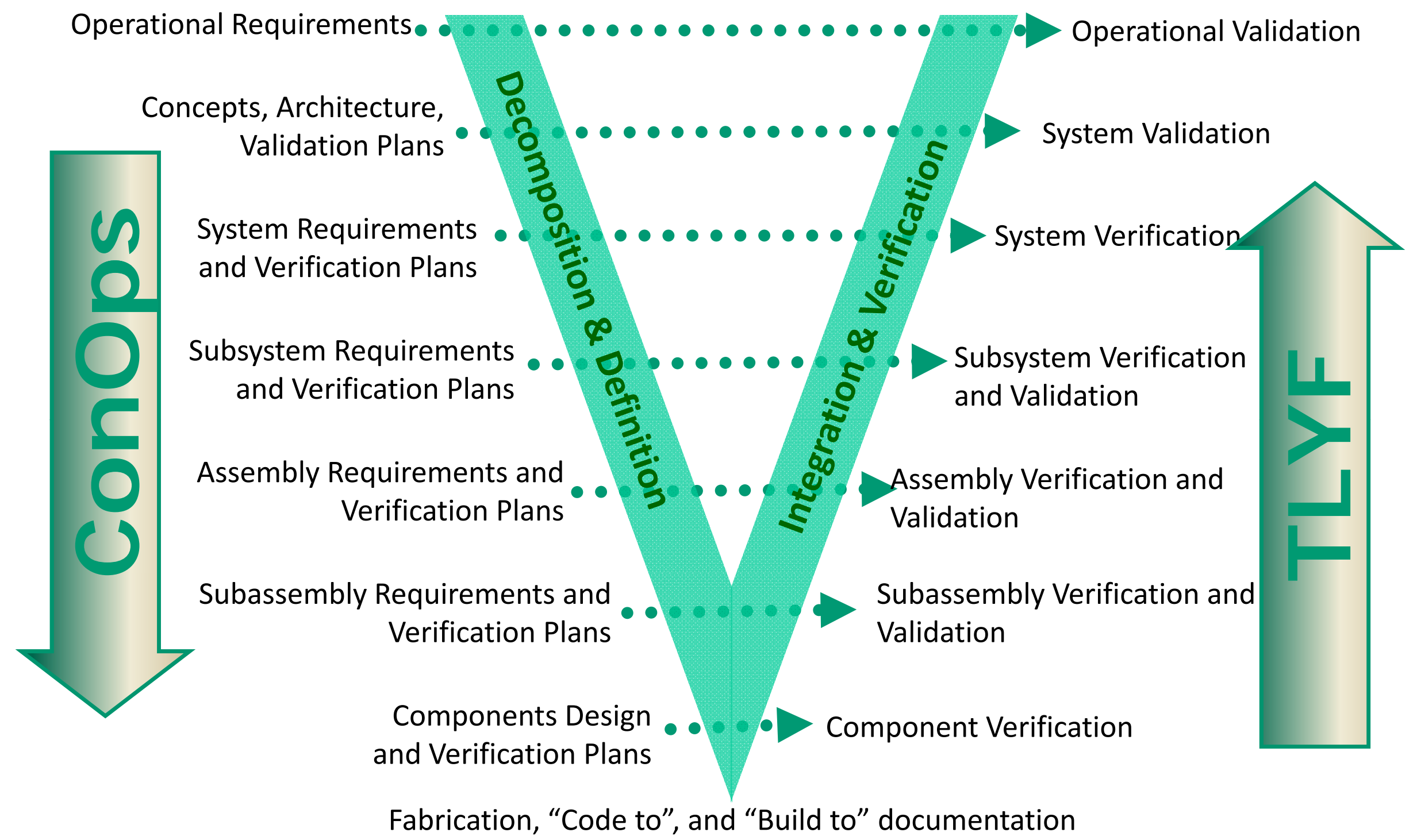




\section{Validation and Verification}

- Product validation

- Confirm that the as-built item can support the mission plan

- Validation has an independent and/or user's perspective

- "Build the right thing"

- Verification

- Confirm that the as-built item can conform to the documented requirements

- "Build the thing right"

Verification \& Validation Objectively Show

That the Mission Can be Achieved 


\section{Validation}

- Requirements validation

- Assure requirements and flow down are what is intended and needed

- Product validation involves system level activity

- Verification may be performed at lower level but need to assure entire product works as intended in the end state and after environments

- Non-flight validation

- Assure tools are correct

- Models, simulators, and test beds

- Must have the correct information for validation

- Implied requirements are not acceptable

\section{Validation is NOT Just for Software}




\section{Verification}

- MUST! determine, plan and coordinate methods early

- To establish scope of program

- To establish expectations with suppliers and customer

- Objectively show compliance that all requirements are fulfilled

- Provide proof of concept

- Verification methods need to be executed, documented, and accepted

- Prior to acceptance of incoming items and

- Prior to customer delivery

- Verification method decisions should be made with quality of proof in mind

\section{Guilty Until Proven Innocent}




\section{Did I Meet the Requirement?}

There must be necessary and sufficient proof that the requirement criteria is met

- Necessary implies that the condition must be met before further evidence is supplied

- Sufficient implies that all appropriate proof is available

- It is necessary that the solar array deploy properly to verify that the power system can provide adequate power to the vehicle, but it is not sufficient

- Tolerances need to be stated to allow variance

- Pass implies that all required aspects are met

- Doing a test or other verification activity is not the same as passing it

- Fail implies that some required aspects are not met

- The requirement is not met if there is any aspect not met

\section{Determine Criteria BEFORE Starting}




\section{VERIFICATION METHODS}




\section{Methods of Verification}
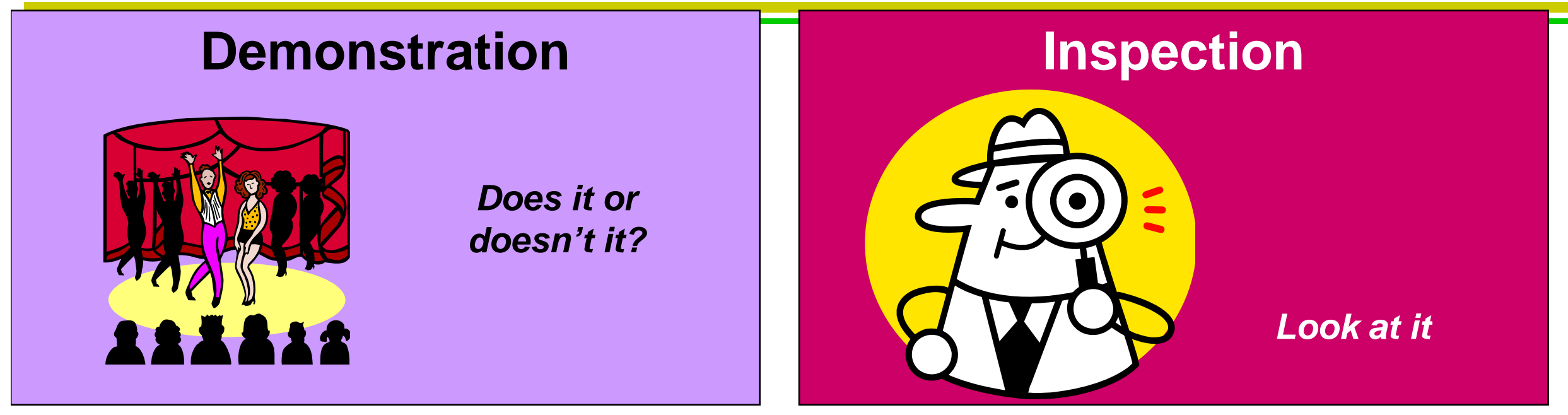

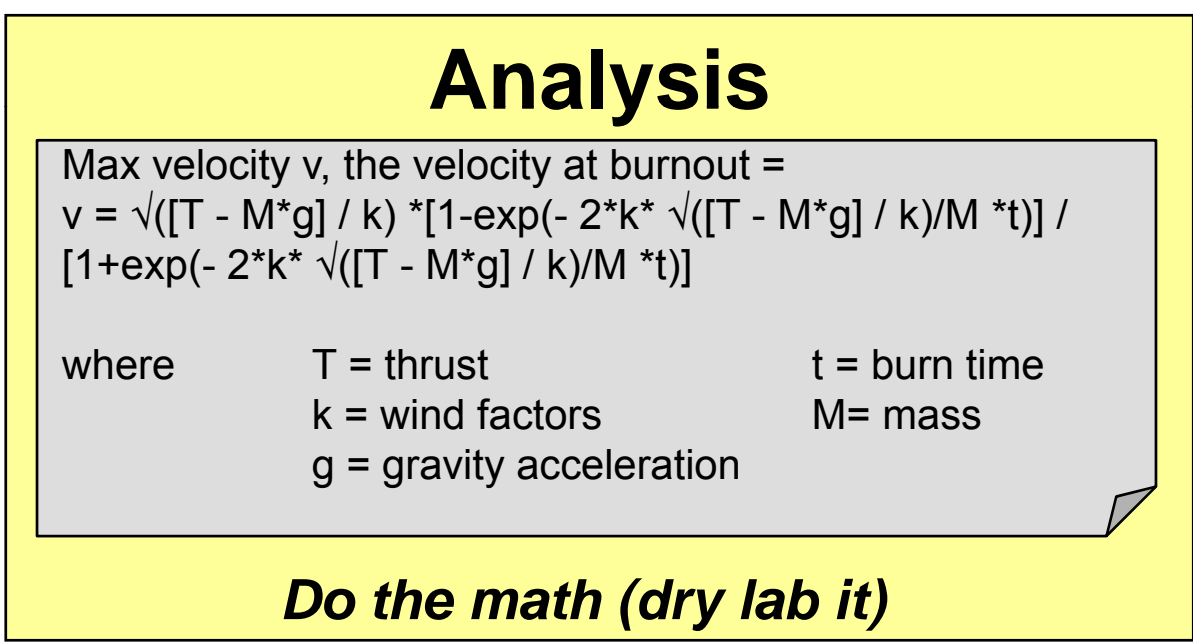
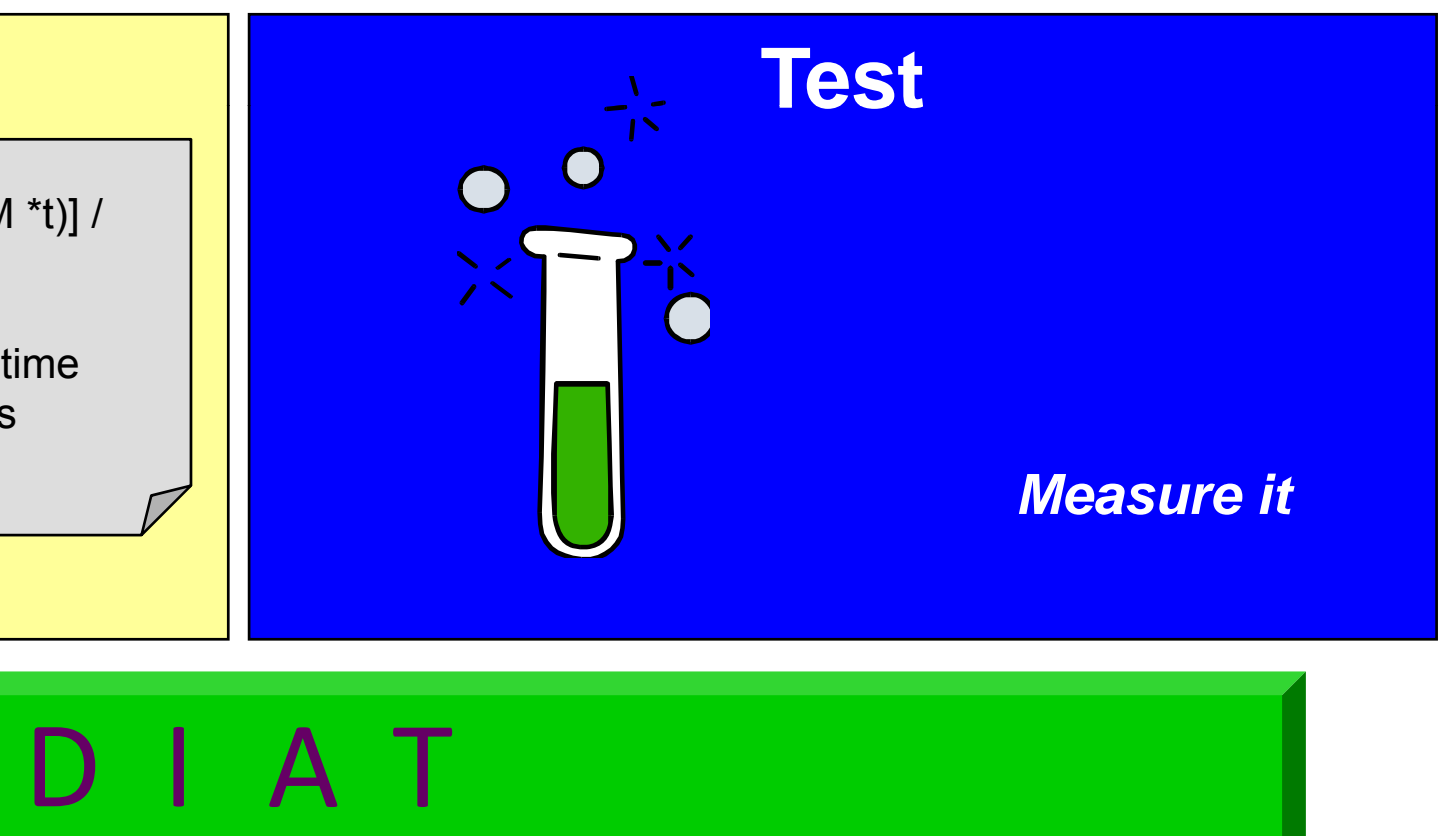


\section{Demonstration}

- Qualitative proof of performance obtained by a successful completion of a pass/fail, go/no go, satisfactory/unsatisfactory or pathfinder event

- Preferred method when

- A proof of concept in a simulated operational environment is required

- An operator (i.e., human interface) is involved

- Often software requirements are verified by demonstrations

- The actual software requirement is inferred to have been successful by virtue of demonstrating a higher level function

Note: This is different than deployment site demonstration / validation (aka "demval")

\section{Qualitative Proof}




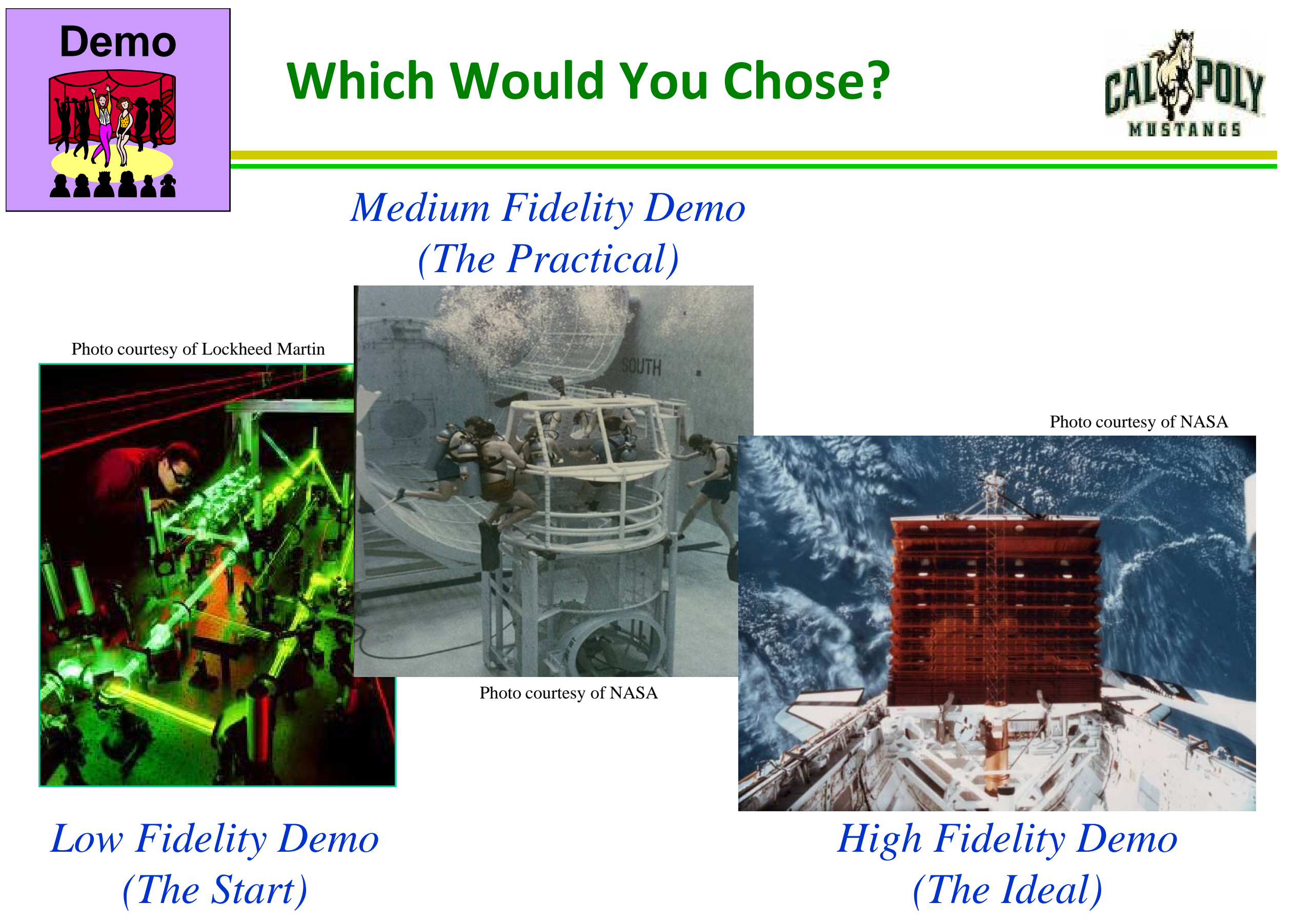




\section{Failures of Demonstration}

2888

- Satellite hits overpass during move damaging shipping container

- Long time between pathfinder and satellite transport

- Road repaved - decreased clearance by 2-inch

- Incorrect command sent to spacecraft causing 3 month mission outage

- Demonstration performed with different personnel

- Boom caught on blanket thus unable to extend fully

- Demonstration not performed with all blankets

\section{Demonstrate With the Correct Fidelity}




\section{Inspection}

\section{Inspection}

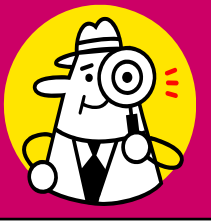

- Physical confirmation of hardware/software characteristics against design documents

- Preferred method when

- Production standards and process control requirements are involved

- Known or high risk design instabilities may exist

- First article inspection (FAI)

- Review of drawings, parts lists, scripts, etc

Note: Production "tests" and "checks" during manufacturing and assembly (such as nondestructive examinations) are classified as inspection

\section{Physical Confirmation - Measurement}




\section{Inspection}

\section{Failures of Inspection}

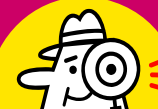

30

- Inspection causes unplanned missile removal

- Boroscope inadvertently trips rail cars due to difficult access

- Incorrectly applied thermal protection tape prevented stage separation stranding satellite in wrong orbit

- What it said: Wrapping should be applied within 0.5 inches of the mounting bracket flange

- What was meant: Wrapping should be applied no closer than 0.5 inches

- "Where's Waldo" syndrome

- Human eye is not perfect if data is cluttered or given too quickly

- Lots of data increases odds of missing something

- Complacency can develop in the inspector

\section{Need Clear and Doable Requirements}




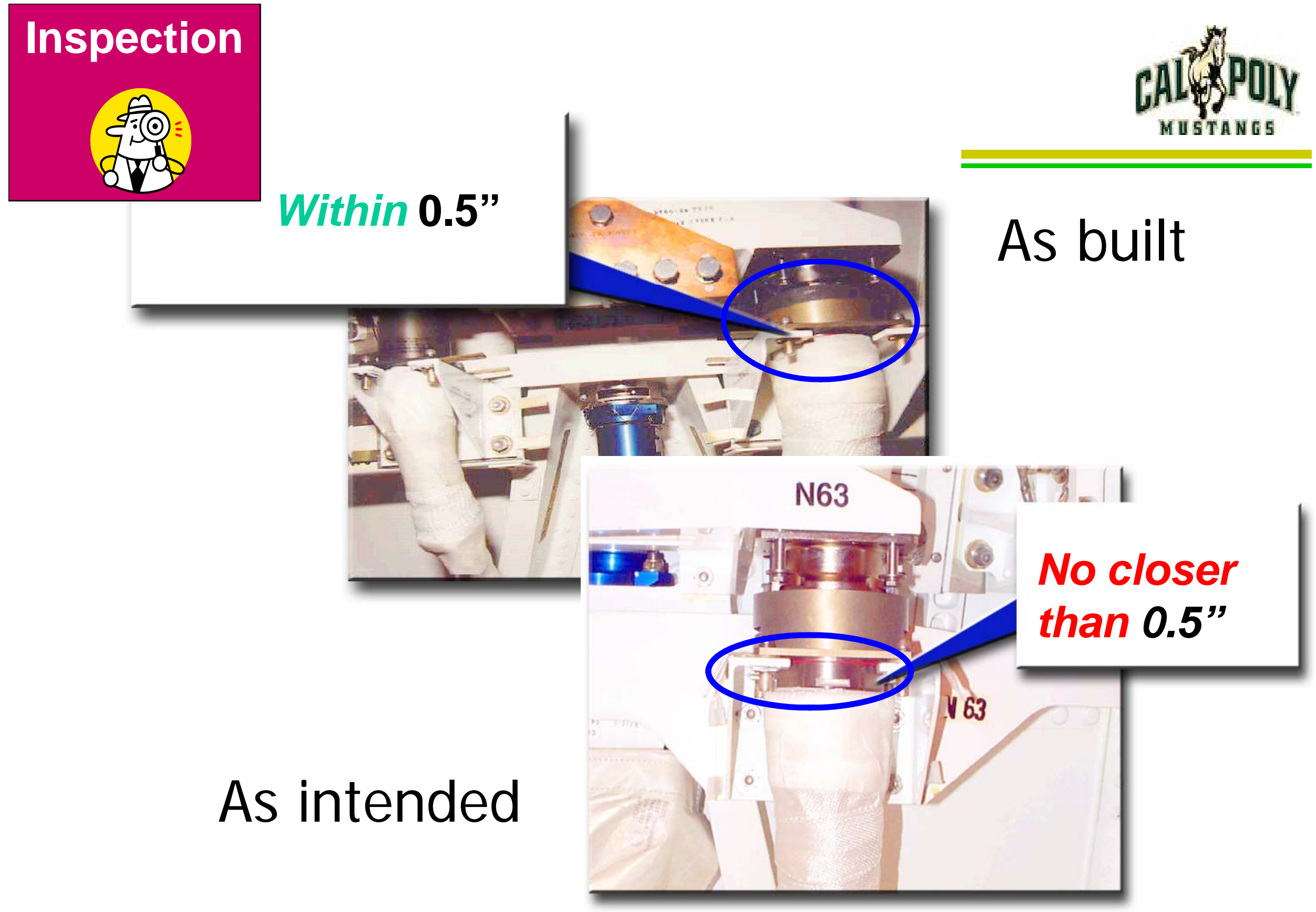




\section{Where's Waldo Syndrome}

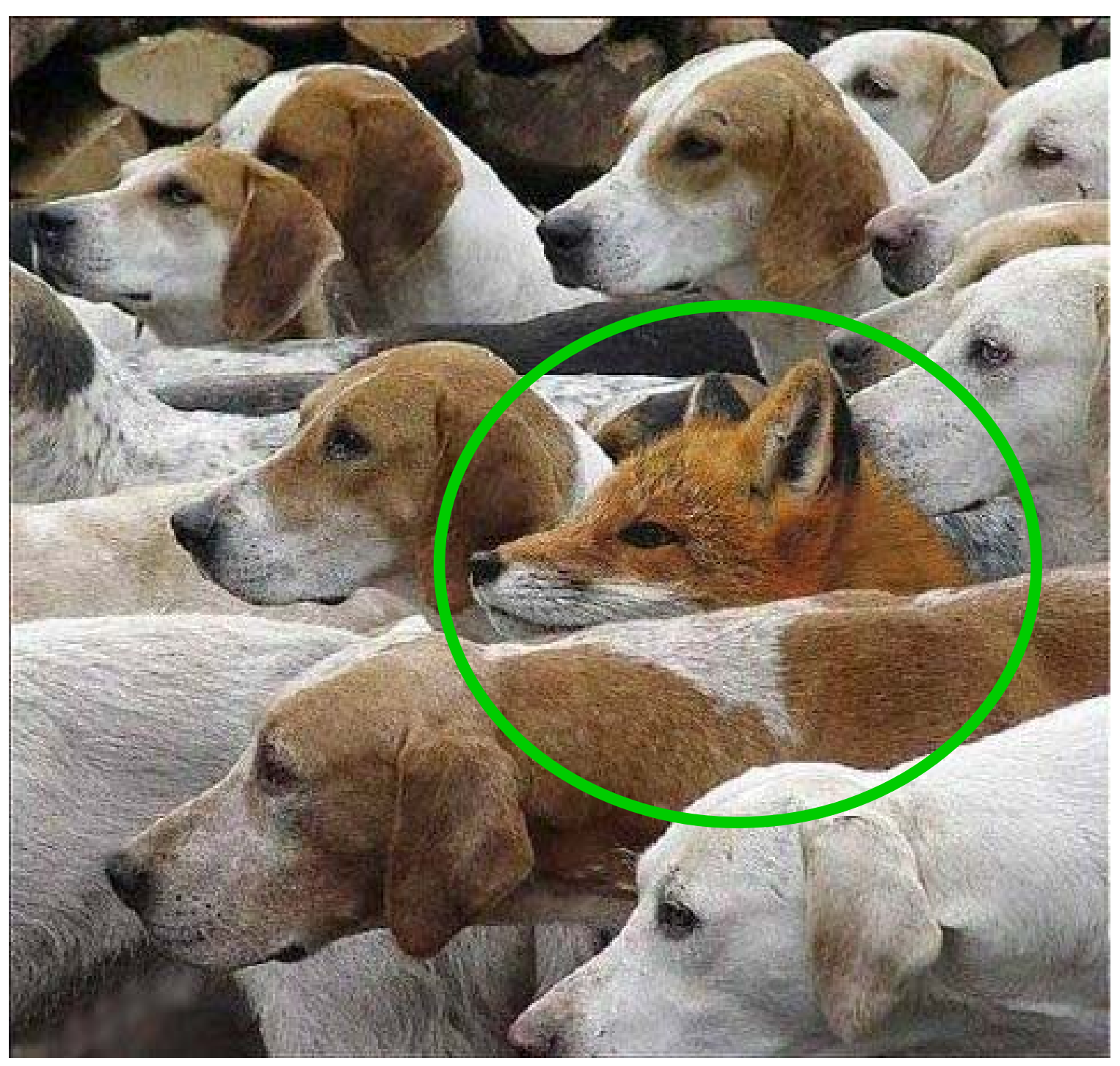




\section{Analysis}

$\Phi v=\sqrt{ }\left(\left[T-M^{*} g\right]\right.$

$\mathrm{F}=\mathrm{ma} \quad \sigma=\mathrm{P} / \mathrm{a}$

\section{Analysis}

- Mathematical or logical treatment of data using appropriate models, simulation, calculations, etc. to reach conclusions that are not directly obtainable from measurements

- Need to validate the model

- Preferred method when

- A heritage (or sufficiently established) design or technology is used

- A test or demonstration is impractical or impossible

- The analytical method is of sufficient fidelity to reflect the specific requirement being verified

- Verifying entire requirements "space" could result in damage to the hardware or software

Note: Analysis includes simulation and similarity

\section{Mathematical Approach}




\section{Analysis}

$\Phi v=\sqrt{ }\left(\left[T-M^{*} g\right]\right.$

$\mathrm{F}=\mathrm{ma} \quad \sigma=\mathrm{P} / \mathrm{a}$

\section{Failures of Analysis}

- Network downlink capability

- Analysis indicated system capable of this with bit error rate $1 \times 10^{-6}$ or better

- Validation test showed analysis off by 3 orders of magnitude or more

- Thermal hardware added at launch base

- Thermal analysis performed early in the design life cycle

- Design changes not adequately assessed

- Solid rocket motor nested too deep and destroyed spacecraft

- Plume heating effects assumptions did not incorporate adequate design margin

- Generic, rather than space vehicle specific, models used

\section{Analysis Must Be Validated}




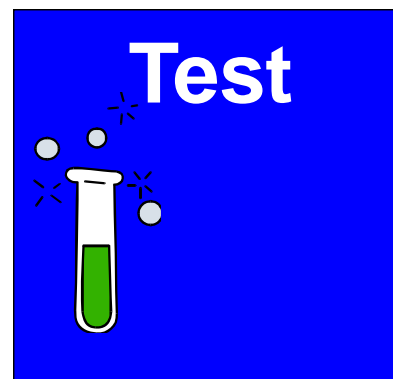

\section{Test}

- Quantitative proof of performance obtained by measurement of a response to a defined stimulus under controlled conditions

- Preferred method

- Must test

- A new (or sufficiently new) design, technology, or application

- Workmanship defects exist that can only be discovered via testing

- Many product to product interactions

\section{The Overall Preferred Method}




\section{Failures of Test}

Test

- Satellite failure undetected during acoustic test

- Passed unpowered acoustic test

- Acoustic environment "shook" electronic package component loose

- Only discovered during thermal vacuum test

- Box level test not perceptive enough to determine circuit inadequacy

- Box successfully passed qualification and acceptance test

- On-orbit (cascaded) failure showed components voltage/current higher than specification levels

- Critical telemetry and command (T\&C) failover missed

- Unit tests proved both T\&C processors worked

- System test proved they could nominally work together

- Did not test failover to redundant side

- Resulted in hung bus without anyway to recover during end to end test

\section{Won't Find the Problem Unless You Test for It}




\section{When It Makes Sense to Combine Methods}

- Some requirements can't be fully verified by one method due to inherent limitations in the method or constraints

- Especially for high level requirements

- Analysis and Test are the most common methods combined to prove the whole

- Reality is inspection, demonstration, and test are done together all the time
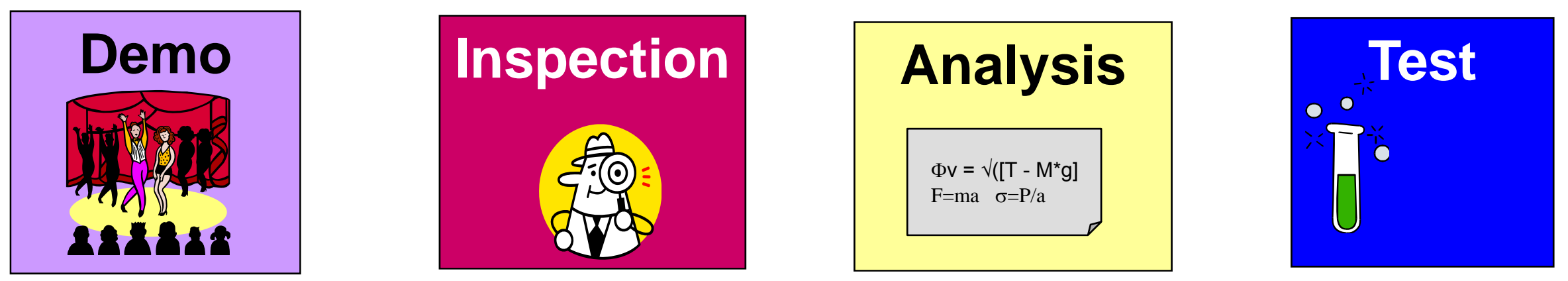


\section{Coupled Loads: Analysis + Test}
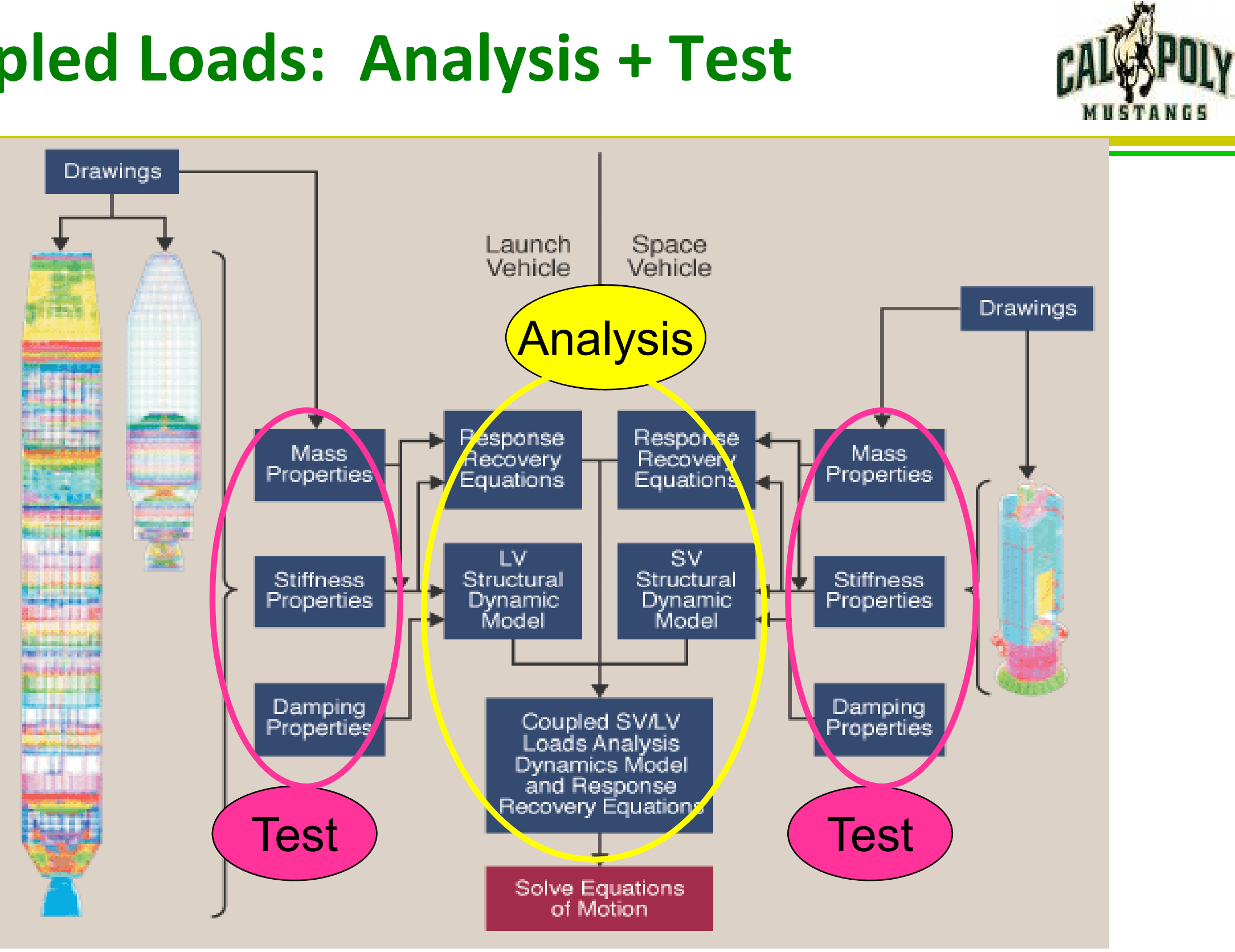


\section{Requirements Verification}

Determine how to verify - requirement by requirement

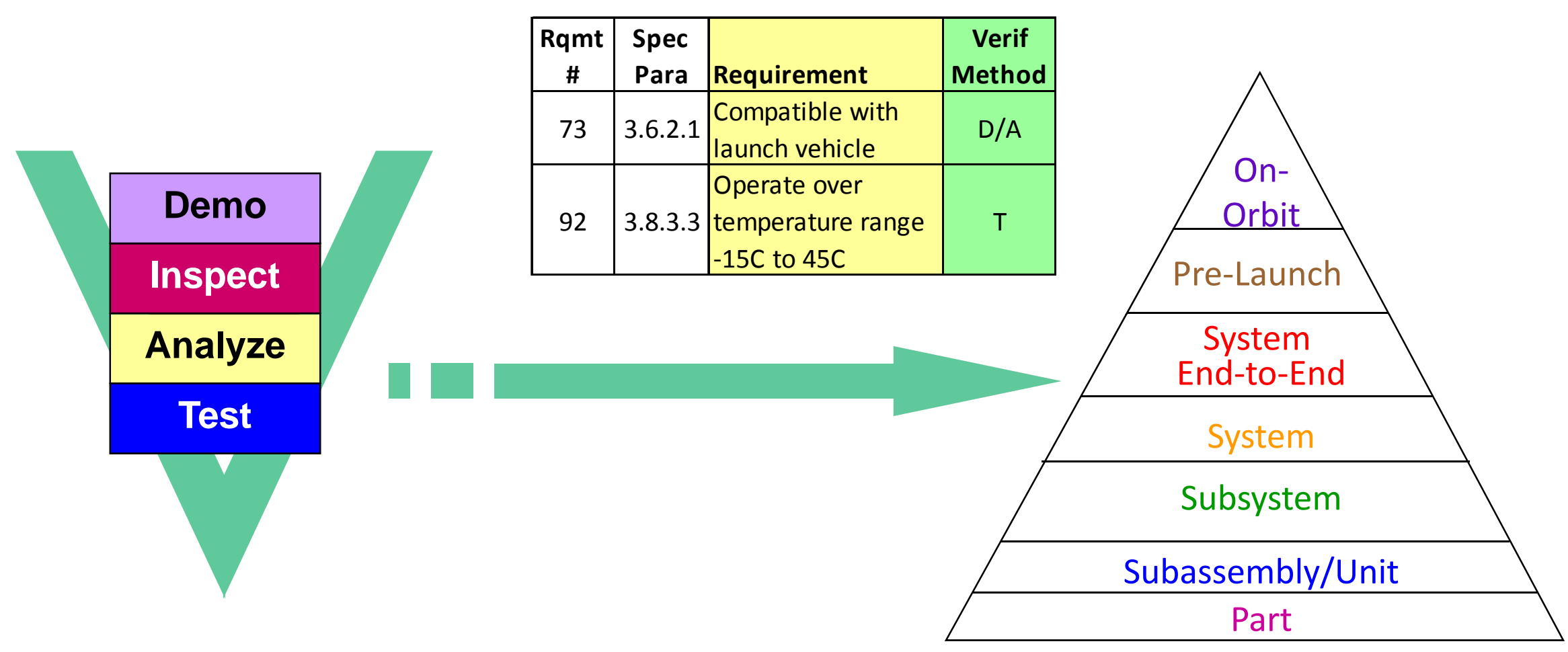

\begin{tabular}{|c|c|l|c|}
\hline $\begin{array}{c}\text { Rqmt } \\
\text { \# }\end{array}$ & $\begin{array}{c}\text { Spec } \\
\text { Para }\end{array}$ & Requirement & $\begin{array}{c}\text { Verif } \\
\text { Method }\end{array}$ \\
\hline 73 & 3.6 .2 .1 & $\begin{array}{l}\text { Compatible with } \\
\text { launch vehicle }\end{array}$ & $\mathrm{D} / \mathrm{A}$ \\
\hline 92 & 3.8 .3 .3 & $\begin{array}{l}\text { Operate over } \\
\text { temperature range } \\
-15 \mathrm{C} \text { to } 45 \mathrm{C}\end{array}$ & $\mathrm{T}$ \\
\hline
\end{tabular}

\section{Verification Process Rolls it All Up}


TEST PHILOSOPHY 


\section{Why Do We Test?}

- Product integrity

- Validate design

- Verify requirements

- Understand performance and margin

- Find defects before flight

- Find the unknown unknowns

- Assure workmanship

- Create confidence

- Certify readiness

- Systems getting increasingly complex

- Interactions not easily modeled

\section{Need Thorough, Disciplined, Perceptive Test that is Representative of Operations}




\section{Test Philosophy}

- Test philosophy is evolving

- Industry is moving from low risk to "managed risk" atmosphere

- Affordability

- Perceptiveness

- Qualify and verify at lowest level

- Screen defects before integrating into next higher assembly

- Most perceptive testing

- Lower repair cost

- Not in single line flow

- Minimize retest

- Next higher assembly

- Validation of requirements

- Verification and qualification of interfaces

- Verification and qualification of performance and functionality

\section{Finding Defects Requires Multiple Levels of Robust Testing}




\section{Levels of Integration}

- Part - single or joined pieces which are not normally subject to disassembly without destruction or impairment of the design use

- Examples: resistor, integrated circuit, relay, roller bearing

- Subassembly/Unit - a functional item that is viewed as a complete and separate entity for purposes of manufacturing, maintenance, or record keeping containing two or more parts which is capable of disassembly or part replacement

- Examples: printed circuit board with parts installed, gear train

- Subsystem - assembly of two or more functionally related subassemblies/units and may include interconnection items such as cables or tubing, and the supporting structure to which they are mounted

- Examples: electrical power, attitude control, telemetry, thermal control, propulsion

- System - integrated set of subsystems capable of supporting an operational role in space

- Examples: space vehicle, launch vehicle, ground station

- System of systems - integrated individual systems to achieve unique, unified, additional capabilities

- Examples: Constellation including ground stations

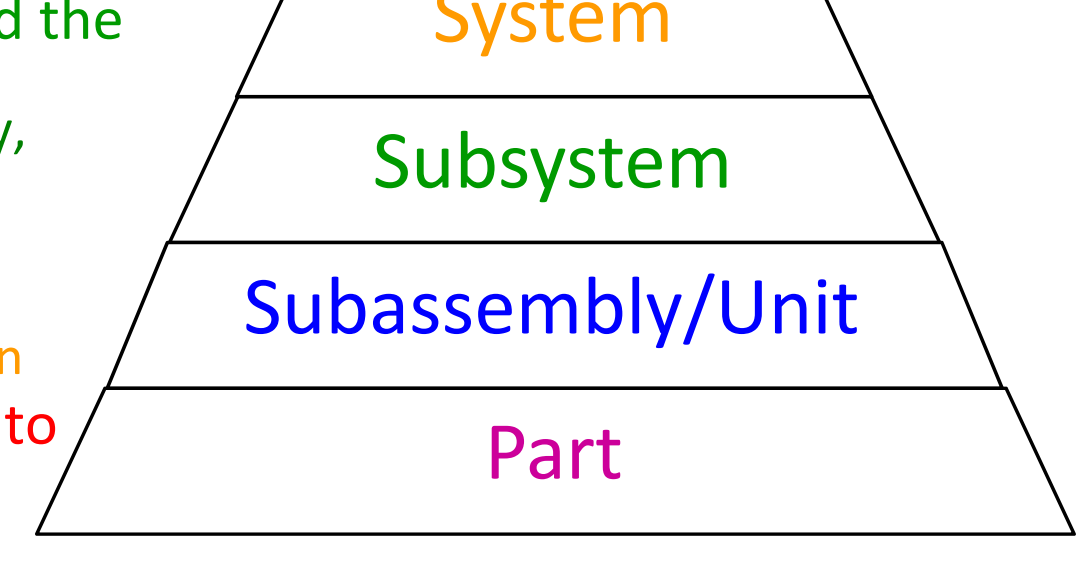




\section{Level of Integration}

Unit

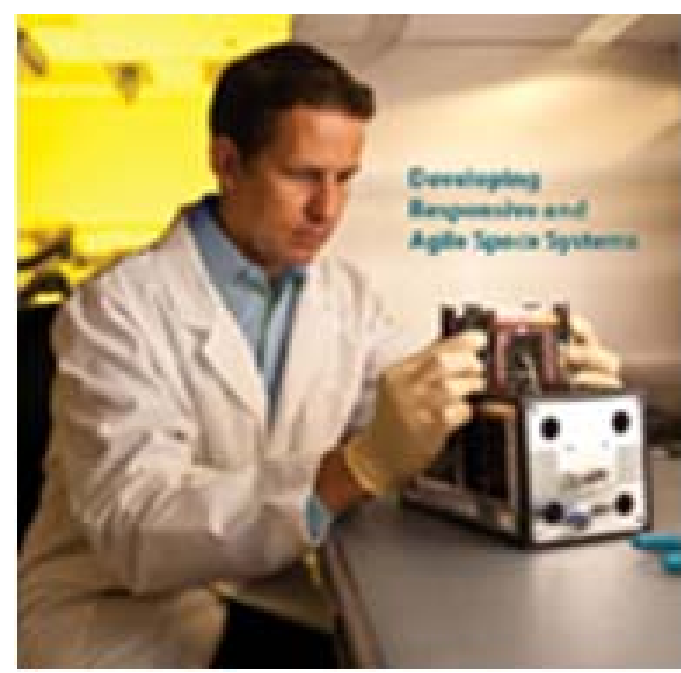

Subassembly
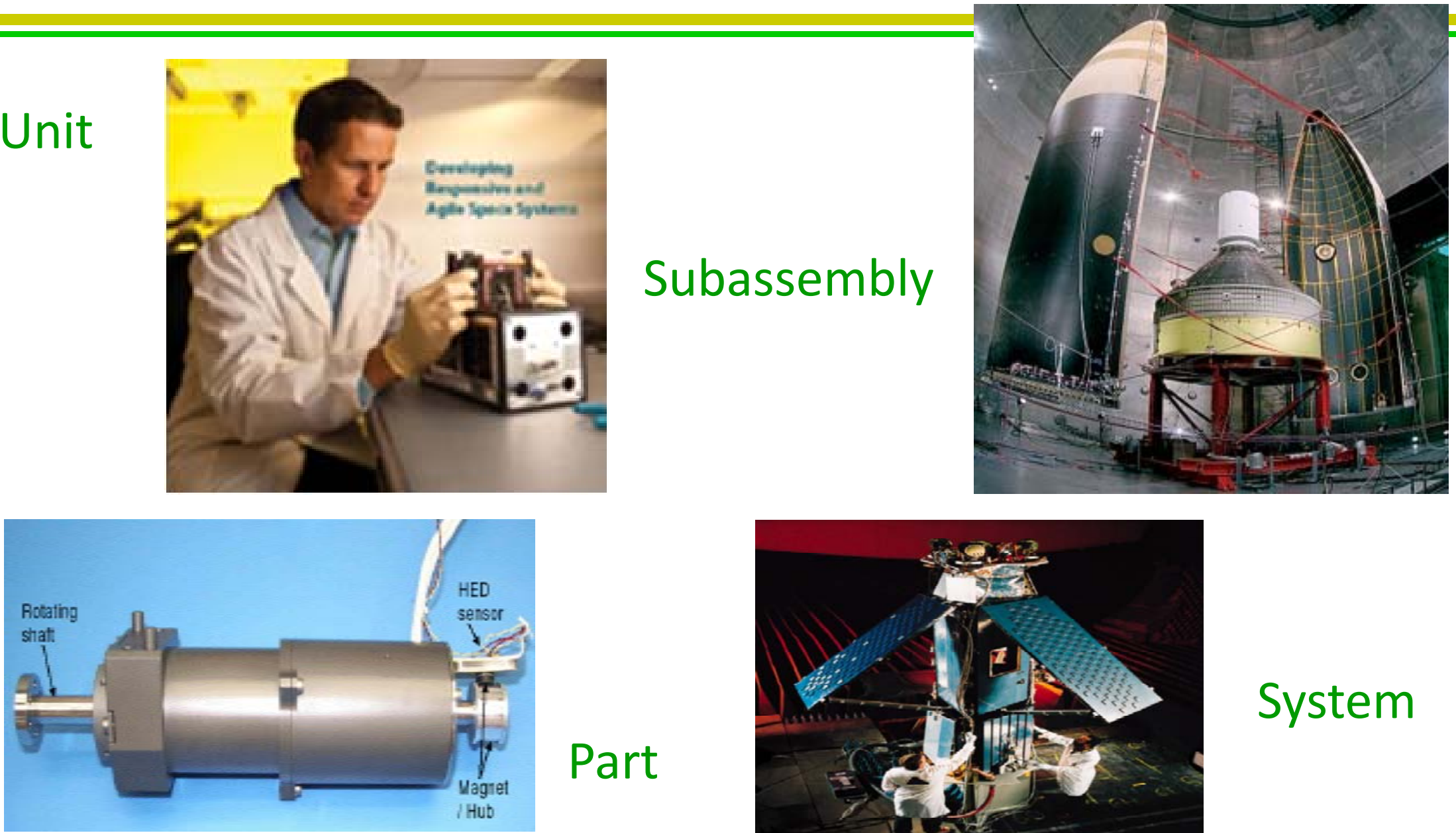

Part

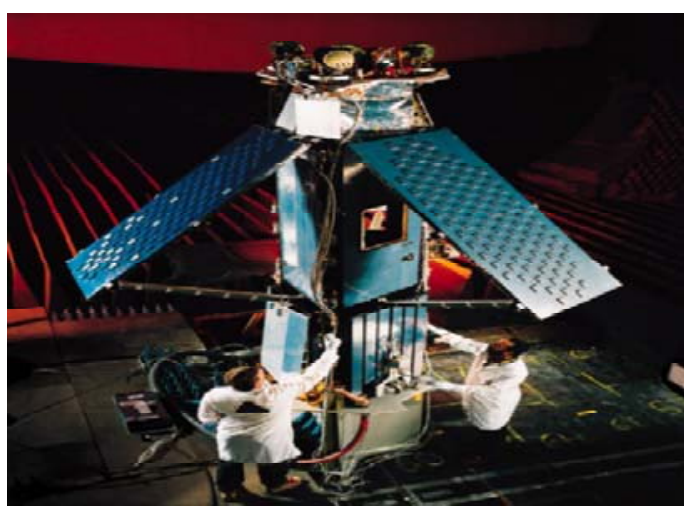

System

\section{Need Correct Verification at Correct Integration}




\section{Test Pyramid}

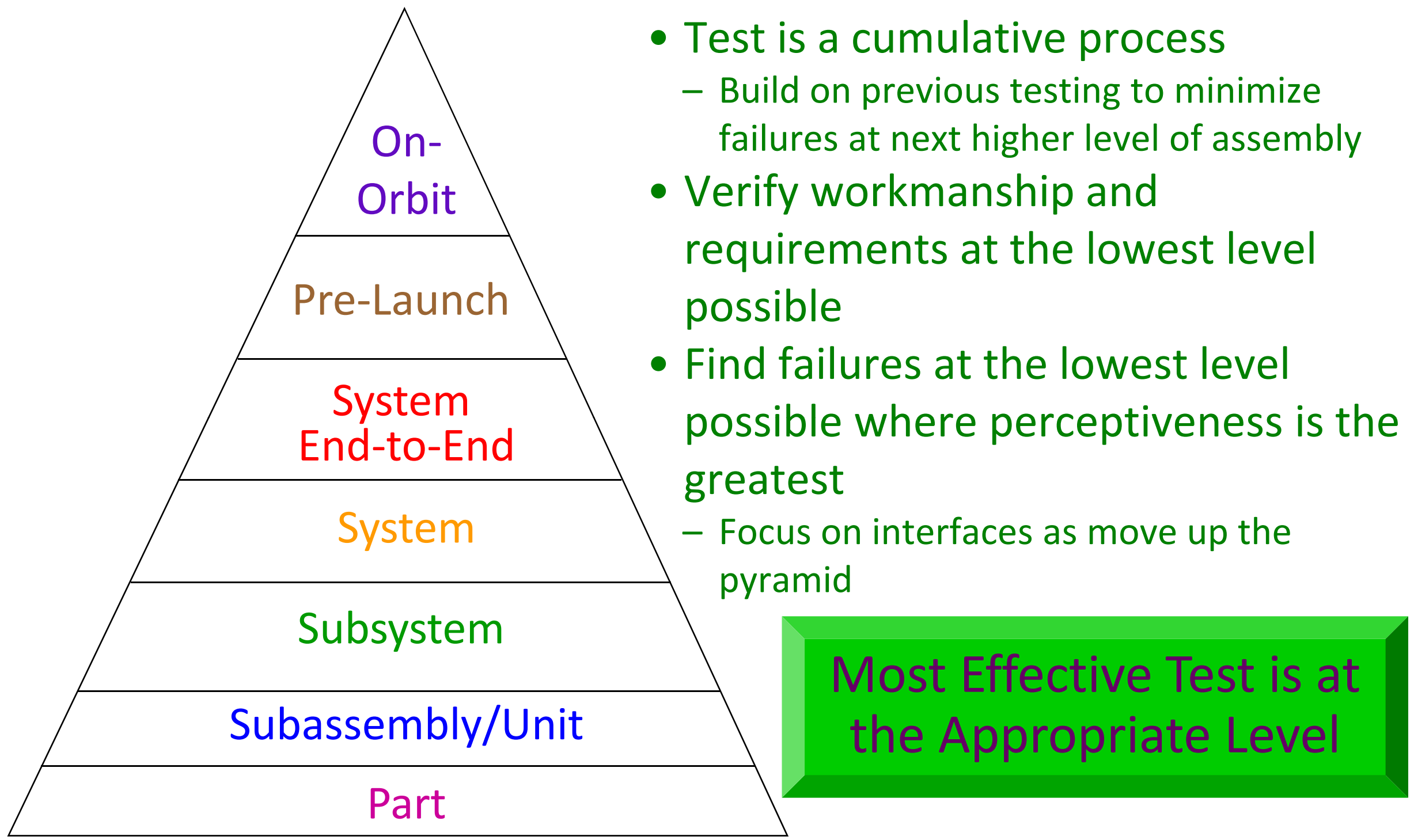




\section{Basics of Test}

- What can be tested

- Anything that can be instrumented to indicate state or numeric value of parameters: hardware, software, databases

- Provide the worst case and nominal conditions on the flight hardware and software

- Understand limitations and impacts of non-flight articles

- Not everything can be tested but do the best you can

- Decide what is NOT done and understand the associated risks and impacts

- Supplement with other verification methods

- What can't be tested, tested well, or tested feasibly

- Where the necessary conditions of the test are physically impossible or difficult to engineer without introducing too much uncertainty in the results or risk to the unit under test (UUT)

- Where the ramaifications of the test vastly exceed the nature of the test 


\section{Test Selection Process}

\section{Approach 1:}

Start with all tests and delete when knowledge allows

\section{Approach 2:}

Start with minimum set and add as experience demands

- Dilemma - Cost and schedule impacts in either case may be prohibitive

- Considerations

- Vibration: all units

- Shock: if flight levels $>0.8 x$ frequency

- Thermal vacuum: if high power and/or thermal dissipation

- Thermal cycle: if low power and/or not sensitive to vacuum

- Acoustic: if large surface

- Leakage: only if pressurized/sealed

- Pressure: if sealed and/or pressure vessel

- Acceleration: only if function affected

- EME: only on electrical

- Life: only if cycled

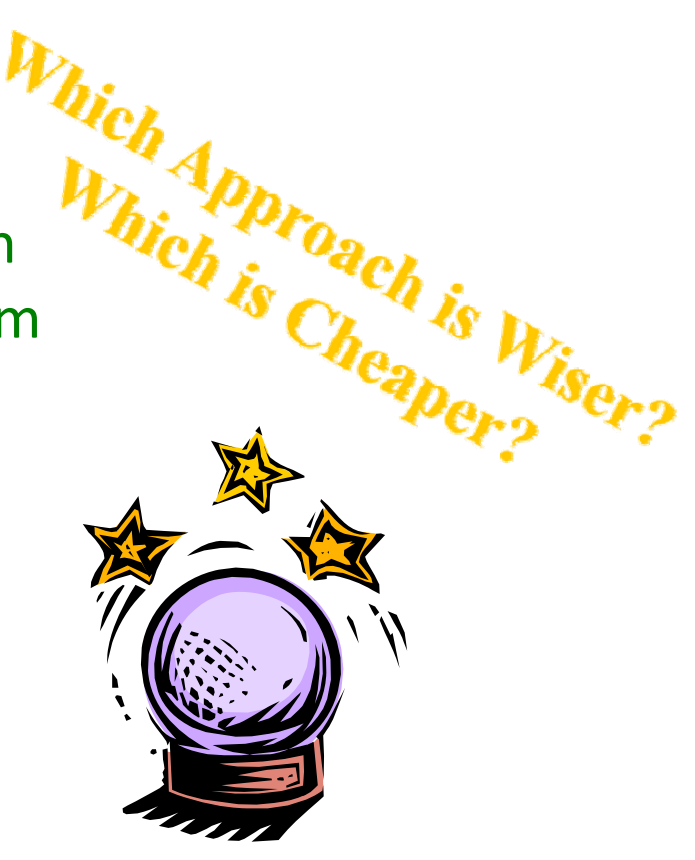

\section{Trust But Verify}




\section{Test Planning}

- A good test program is one where the hardware performance is verified under fielded conditions

- Development tests find breaking points

- Qualification tests validate performance design margins

- Acceptance tests validate hardware is free of workmanship defects and will perform under fielded conditions

- Decisions to be made

- Desired performance margins

- Desired tests to screen for workmanship defects

- Validate performance before, during and/or after exposure 


\section{Test Planning (cont)}

- Software test planning must be incorporated into system test planning during the earliest stages of the system life cycle

- Levels of software and system integration for space and ground

- Integration levels where requirements will be verified

- Software, hardware, and system test planning include planning for development or procurement of necessary test resources

- Simulators, emulators

- Test drivers, stimulators

- Automated test execution tools

- Automated Test Equipment (ATE)

- Automated Ground Equipment (AGE)

\section{Early and Thorough Test Planning is Essential}




\section{Development Tests}

Tests conducted on representative articles to

- Validate/demonstrate the evolution of designs from the conceptual phase to the operational phase

- New design concepts

- Application of proven concepts and techniques to a new configuration

- Technology insertion

- Characterize engineering parameters, gather data

- Reduce risk involved in committing designs to the fabrication of qualification and flight hardware

- Breadboard testing

- Model validation

- Validate test scripts and procedures

- Investigate problems or concerns that arise after successful qualification

- Engineering characterization tests and tests to validate qualification and acceptance procedures 


\section{Qualification Tests}

- Demonstrate that the design, manufacturing process, and acceptance program produce mission items that meet specification requirements

- Validate the planned acceptance program including test techniques, procedures, equipment, instrumentation, and software

- Performed beyond maximum predicted environments (MPE) to provide margin

- A single qualification test item of a given design should be exposed to all applicable environmental tests

- Protoqualification tests (protoqual/protoflight)

- Modified qualification (reduced margin) conducted on product which is considered to be available for flight

- Saves costs but increases risk

- Protoflight tests all units

- Protoqual tests only the first unit; subsequent units tested at acceptance levels 


\section{Acceptance Tests}

- Demonstrate the acceptability of each deliverable product

- Demonstrate conformance to subset of specification requirements

- Provide quality-control assurance against workmanship or material deficiencies

- Intended to stress screen items to precipitate incipient failures due to latent defects in parts, materials, and workmanship

- Performed at MPE

- Will not create conditions that exceed appropriate design safety margins or cause unrealistic modes of failure 


\section{Test Like You Fly (TLYF)}

- There shall be no nominal activity will be performed in operation that hasn't been demonstrated in a flight-like manner on the ground

- Don't want first occurrence of operation to be experienced in space

- Test in same order and same conditions like it is used (flown)

- Recommended approach to verification and validation and associated risk decision making

- Involves system engineers, hardware and software engineers, test creators, mission designers, operations personnel, and those charged with independent evaluation of design verification

- Provides a unique assessment process that focuses on determining the "mission-related" or "like you fly" risks associated with potential flaws in our space systems

\section{Test Like You Fly !!}




\section{Not Like You Fly}

- Frequently, it is not possible to test like you fly

- Those limitations (aka exceptions) are due to

- Physics (can't be done)

- Engineering (not practical to do)

- Programmatic (philosophy, cost or schedule constraints)

- TLYF limitations shall be addressed with risk assessment and mitigation

- Mitigation can be adjunct analyses, non-TLYF tests, etc.

- Often mitigated by a "sum of the parts" approach

- Risk of doing test in a flight-like way must be weighed against the risk of missing flaws when doing it in a non-flight-like way (or not doing it at all)

\section{Need to Understand the Risk and Impacts}




\section{Test Resources}

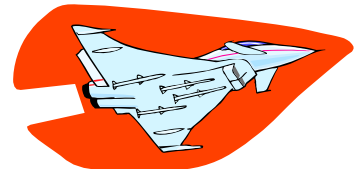

- Test Articles

- Preferably in matrix format, show key government and contractor resources

- Test Sites and Instrumentation - Ranges and national facilities

- Test Support Equipment - Special GSE that must be acquired

- Threat Systems/Simulators

- Type and fidelity

- Test Targets and Expendables

- Rockets, bombs, target characteristics, etc.

- Operational Force Test Support

- Planes, ships, on-orbit assets alre under assignment

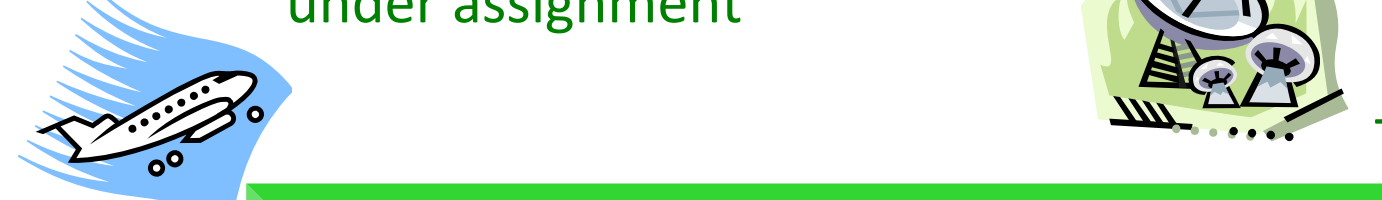

- Simulations, Models and Testbeds

- Computer driven simulators for hardware-in-the-loop type system testing

- Special Requirements

- Any significant non-instrumentation capabilities and resources such as: unique geodesy products or restricted air/sea/landscapes

- Test and Evaluation Funding Requirements

- Estimate, by fiscal year and appropriation line number

- Manpower/Personnel Training

- Training requirements and limitations that affect test and evaluation execution

- Human factors

\section{The Earlier You Determine Test Program Needs - The Better}




\section{Not for Flight Hardware: Brassboards, Engineering Units, Test Beds}

- Brassboards

- A circuit board or unit using flight equivalent, but not flight qualified, parts and assembly techniques

- Used as functional and interface test article

- Engineering Unit

- A flight equivalent, and possibly flight qualifiable, unit meant to be used as a test article in a test bed

- Test Beds

- An electrical and signal flight equivalent assembly of a subsystem or satellite using brassboard units. May not be mechanically equivalent to satellite in layout

- Used to perform initial subsystem and/or spacecraft hardware and software functional tests; validate test procedures for flight units; troubleshoot problems identified on flight spacecraft both during ground test and on-orbit operations

- Stress testing

- Off-nominal testing

These Non-Flight Items Can Be Very Handy... But Often First on the List to Be Deleted 


\section{Maybe Not for Flight Hardware}

- Structural test model

- Form/fit precursor to flight model

- Tested to qualification levels of vibration, shock, acoustic

- Can be used to verify harness layout, interference paths, fit checks, etc.

- Simulators

- Simulates specific conditions or the characteristics of a real process or operation

- Potential development / anomaly investigation platform thereafter

- Qualification test hardware

- Flight-like but highly stressed

- Validate manufacturability and design concepts

- Test to qualification levels for all environmental test

- Potential development / anomaly investigation platform thereafter

\section{Non-flight Hardware Can be Upgraded to Flight}




\section{Test Related Issues}

- Minimal planning

- Don't understand the UUT

- Don't have documented steps

- Don't have clear criteria including tolerances

- Incorrect test article fidelity

- Test not perceptive enough

- Not Test Like You Fly

- Margins unknown or not understood

- All the modes and states are not known or addressed

- Not understanding impact of UUT vs flight configurations

- Environments or conditions not properly coupled

\section{Unclear Unknown Undisciplined}


TEST OBJECTIVES 


\section{Integration of Test}

\begin{tabular}{|c|c|c|c|c|c|}
\hline 2 & 冨易 & 共量 & & 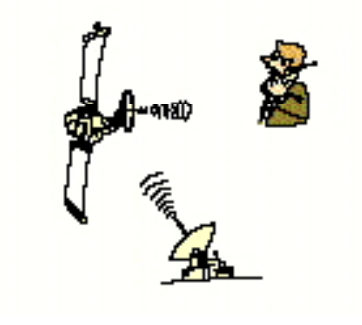 & s. \\
\hline Parts & $\begin{array}{c}\text { Subassembly / } \\
\text { Unit }\end{array}$ & $\begin{array}{l}\text { Subsystem } \\
\text { Integration }\end{array}$ & $\begin{array}{l}\text { System: Payload } \\
\& \text { Vehicle } \\
\text { Integration }\end{array}$ & $\begin{array}{l}\text { System } \\
\text { End-to-End }\end{array}$ & $\begin{array}{l}\text { Pre-Launch } \\
\text { Validation }\end{array}$ \\
\hline \multirow[t]{4}{*}{$\begin{array}{l}\text { - Electrical } \\
\text { - Thermal } \\
\text { - Vibration } \\
\text { - X-ray } \\
\text { - Life } \\
\text { - Leak } \\
\text { - Radiation }\end{array}$} & \multirow{4}{*}{$\begin{array}{l}\text { - Functional } \\
\text { - Electrical } \\
\text { - Mechanical } \\
\text { - Software } \\
\text { - Vibration } \\
\text { - Shock } \\
\text { - Burn-in } \\
\text { - Temp cycle } \\
\text { - Vacuum } \\
\text { - Pressure/leak } \\
\text { - EMI/EMC } \\
\text { - Special }\end{array}$} & \multirow{3}{*}{$\begin{array}{l}\text { - Functional } \\
\text { - Interface } \\
\text { Verification } \\
\text { - Electrical } \\
\text { - Signal } \\
\text { - Pressure/leak } \\
\text { - Hardware in- } \\
\text { the-loop } \\
\text { simulation } \\
\text { - Software }\end{array}$} & \multirow{4}{*}{$\begin{array}{l}\text { - Functional } \\
\text { - Interface } \\
\text { Verification } \\
\text { - Electrical } \\
\text { - Mechanical } \\
\text { - Software } \\
\text { - Acoustic } \\
\text { - Vibration } \\
\text { - Shock } \\
\text { - Orbit Thermal- } \\
\text { Vacuum } \\
\text { - Pressure/leak } \\
\text { - EMI/EMC } \\
\text { - Special }\end{array}$} & \multirow{3}{*}{$\begin{array}{l}\text { - Functional } \\
\text { - Signal } \\
\text { - RF } \\
\text { - Electrical } \\
\text { - Mechanical } \\
\text { - Flight SW } \\
\text { - Ground SW } \\
\text { - Procedures } \\
\text { - Databases } \\
\text { - Data Analysis } \\
\text { - Ops Concept }\end{array}$} & \multirow[t]{2}{*}{$\begin{array}{l}\text { - Functional } \\
\text { - Electrical } \\
\text { - Mechanical } \\
\text { - Software } \\
\text { - Pressure/leak } \\
\text { - Install before } \\
\text { flight } \\
\text { - Remove before } \\
\text { flight }\end{array}$} \\
\hline & & & & & \\
\hline & & & & & \\
\hline & & & & & \\
\hline
\end{tabular}




\section{Integration \& Test Flow}

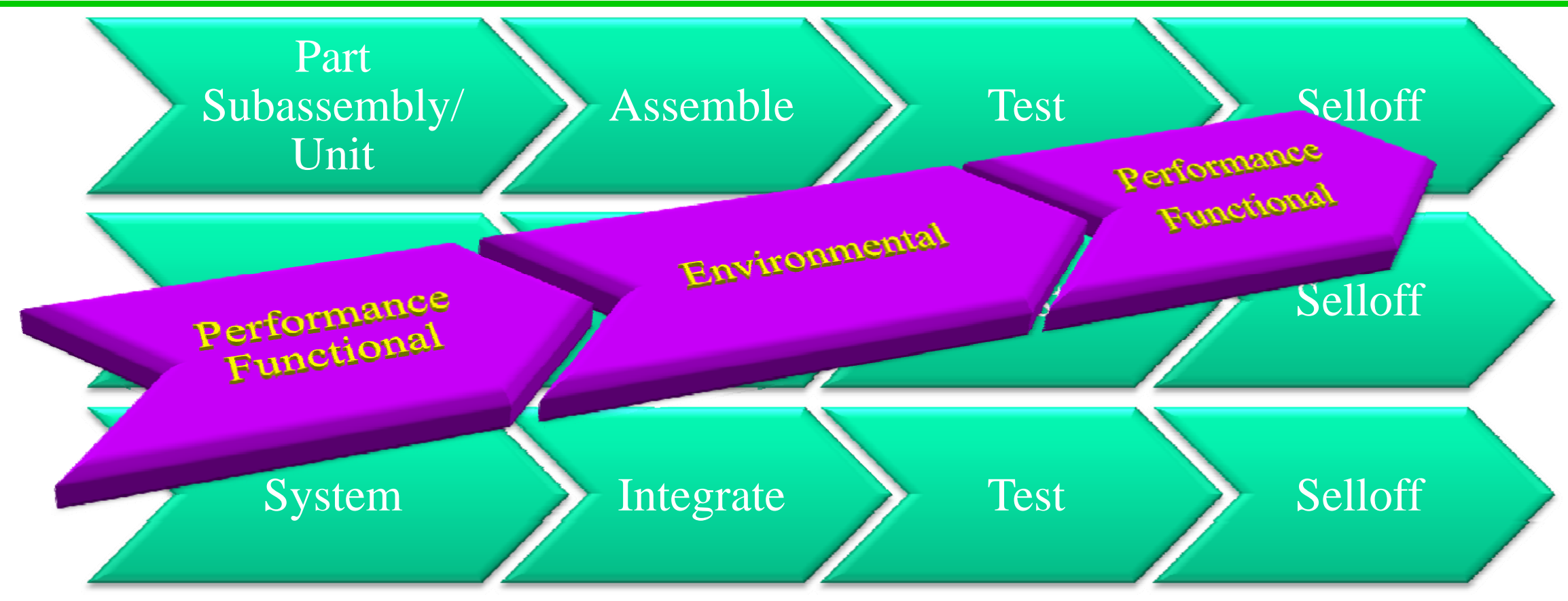

- Performance Testing

- Demonstrate electrical, optical, mechanical, and software of UUT satisfies specification requirements and tolerances

- Demonstrates design margins and specification compliance for all pathways and modes

- Functional Testing

- Assess operability of UUT within boundaries established by design requirements 
Test Objectives - Part

- Definition

- Piece(s) not normally subject to disassembly without destruction or impairment of the design use

- Test Objective

- Screen for workmanship and latent parts defects

- Verify part specification performance
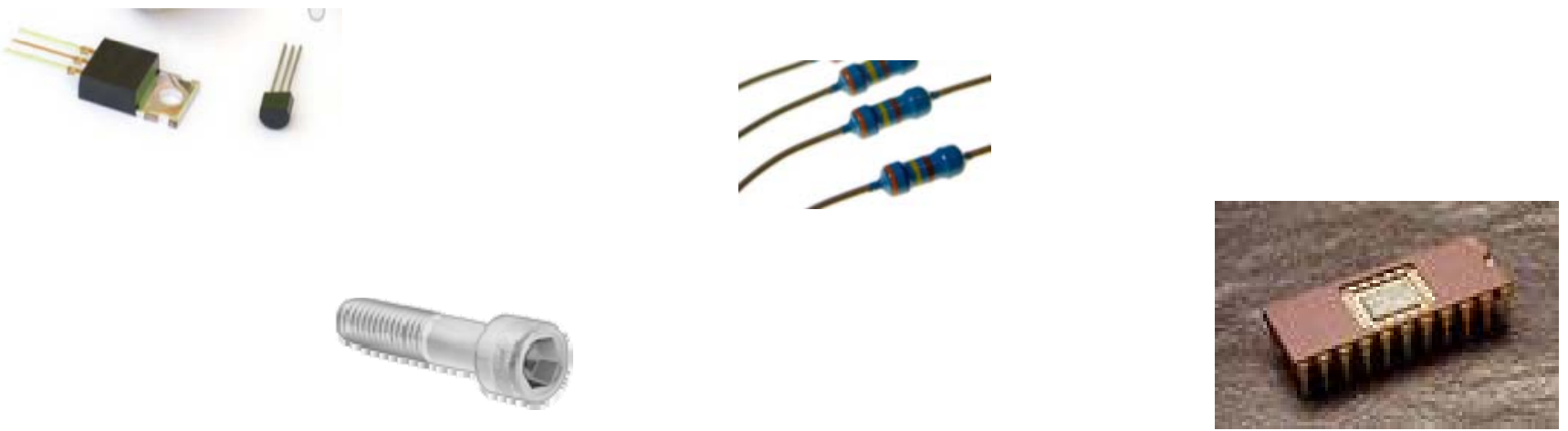

\section{Start with Good Products}




\section{Test Objectives - Subassembly/Unit}

\section{- Definition}

- Unit containing two or more parts which is capable of disassembly or part replacement

- Test Objective

- Verify workmanship and design

- Verify subassembly/unit specification performance

- Can have software and/or firmware

- Perceptive testing at the subassembly level reduces repairs and saves schedule and cost

- LMMS paper 1986, D. A. Smith reviewed 29 units

- 82 Critical failures counted $=$ Failure rate of 2.8 per unit

- 59 of the 82 (72\%) could have been detected at card level

- Computer industry routinely screens populated boards for defects

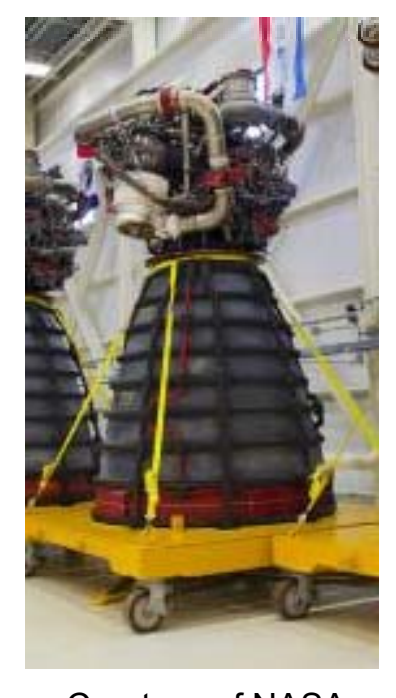

Courtesy of NASA

\section{Perceptive Part and Unit Tests Save $\$ \$$}




\section{Unit Qualification Tests}

\begin{tabular}{|l|c|c|c|c|c|c|c|c|c|c|}
\hline Units & $\begin{array}{c}\text { Electrical } \\
\text { and } \\
\text { Electronic }\end{array}$ & Antenna & MMA & $\begin{array}{c}\text { Solar } \\
\text { Array }\end{array}$ & Battery & $\begin{array}{c}\text { Valve or } \\
\text { Propulsion } \\
\text { Component }\end{array}$ & $\begin{array}{c}\text { Pressure } \\
\text { Vessel or } \\
\text { Component }\end{array}$ & Thruster & Thermal & Optical \\
Components
\end{tabular}

Ref: TR-2004(8583)-1 Rev A (MIL-STD 1540E) Table 6.3-1 


\section{Unit Acceptance Tests}

\begin{tabular}{|l|c|c|c|c|c|c|c|c|c|c|}
\hline Units & $\begin{array}{c}\text { Electrical } \\
\text { and } \\
\text { Electronic }\end{array}$ & Antenna & MMA & $\begin{array}{c}\text { Solar } \\
\text { Array }\end{array}$ & Battery & $\begin{array}{c}\text { Valve or } \\
\text { Propulsion } \\
\text { Component }\end{array}$ & $\begin{array}{c}\text { Pressure } \\
\text { Vessel or } \\
\text { Component }\end{array}$ & Thruster & Thermal & $\begin{array}{c}\text { Structural } \\
\text { Optical }\end{array}$ \\
\hline Components
\end{tabular}

Ref: TR-2004(8583)-1 Rev A (MIL-STD 1540E) Table 6.3-2 


\section{Typical Unit Test Sequence}
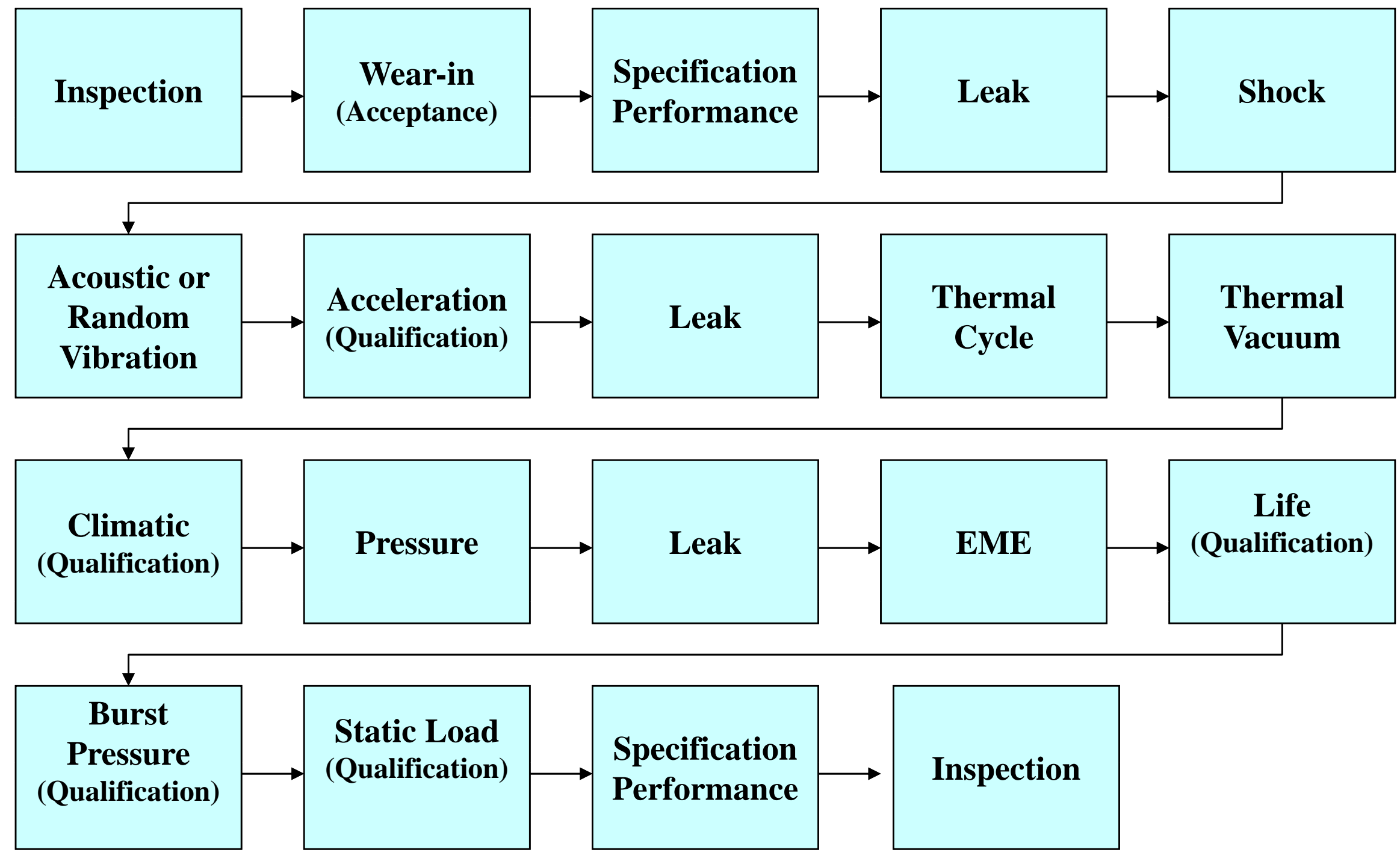


\section{Unit Test Margins and Duration}

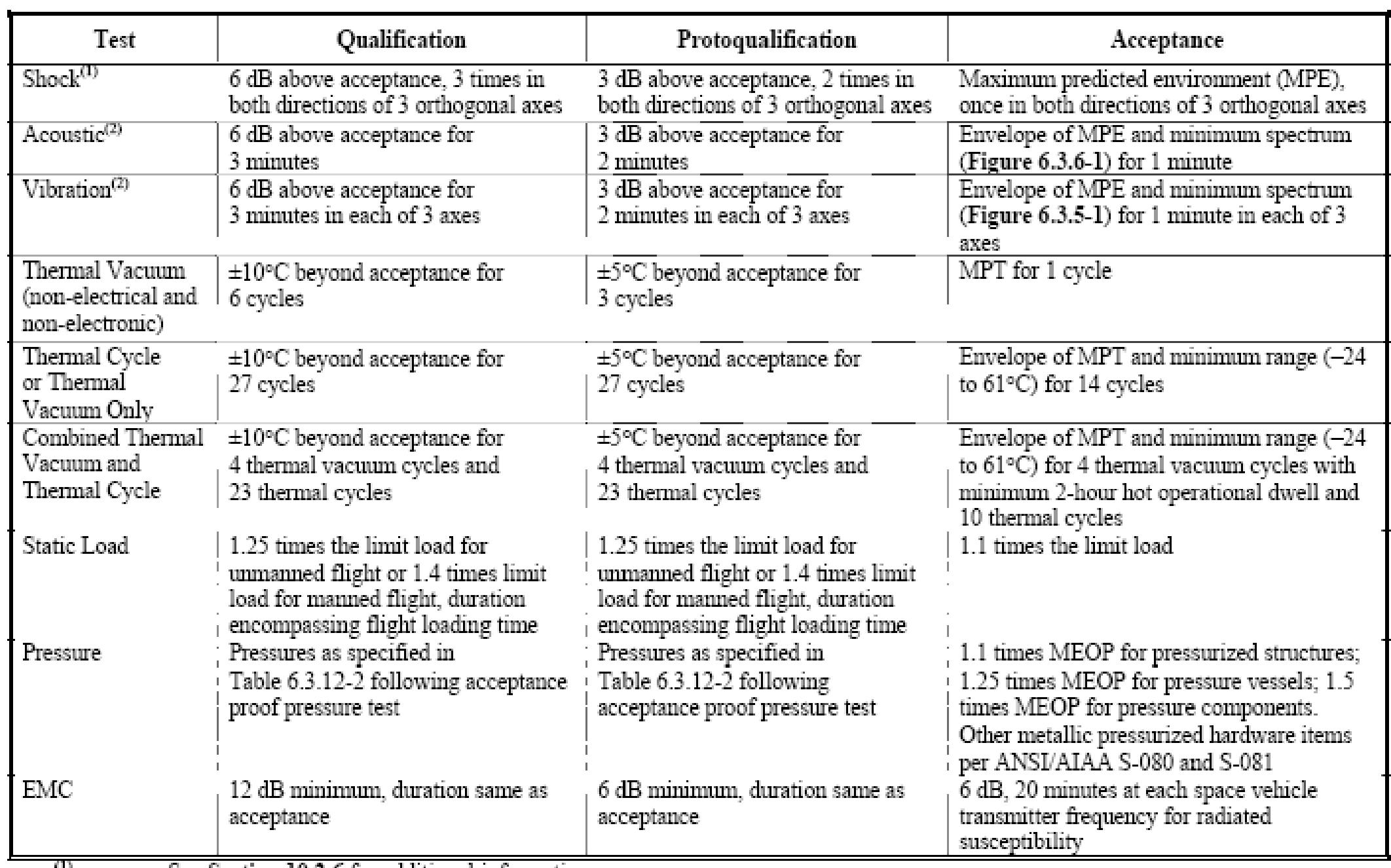

(1) See Section 10.2.6 for additional information.

(2) See Section 10.2.2 and 10.2.3 for units with effective duration greater than 15 seconds.

Ref: TR-2004(8583)-1 Rev A (MIL-STD 1540E) Table 6.3-3 


\section{Space Vehicle Subsystems Integration}

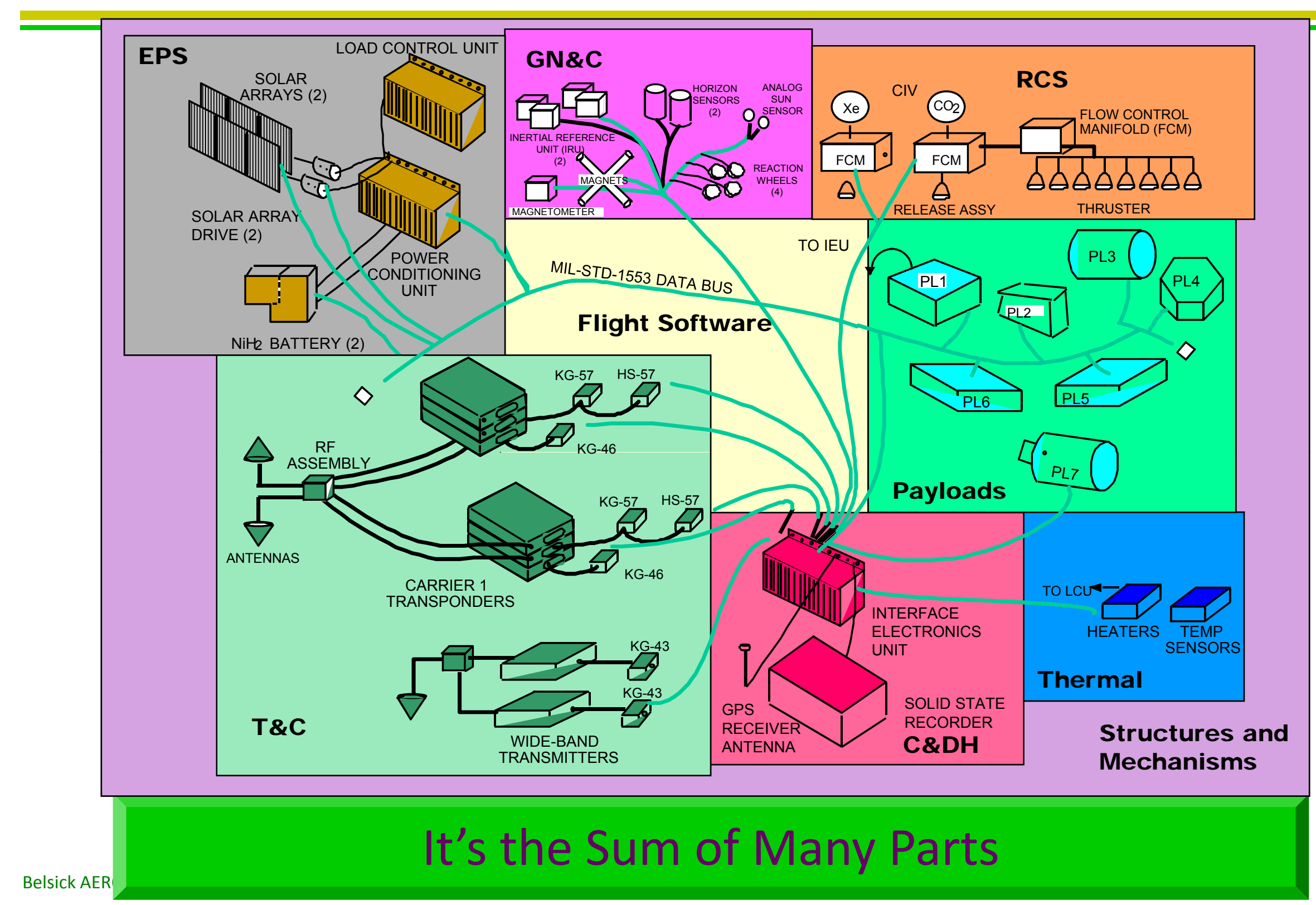




\section{Test Objectives - Subsystem}

- Definition

- Assembly composed of functionally related units that perform one or more prescribed functions

- Test Objective

- Verify interfaces and key performance parameters

- Verify specification performance

- Should have flight software and firmware

- Access often limited at system level

- Provide a more perceptive test than system level

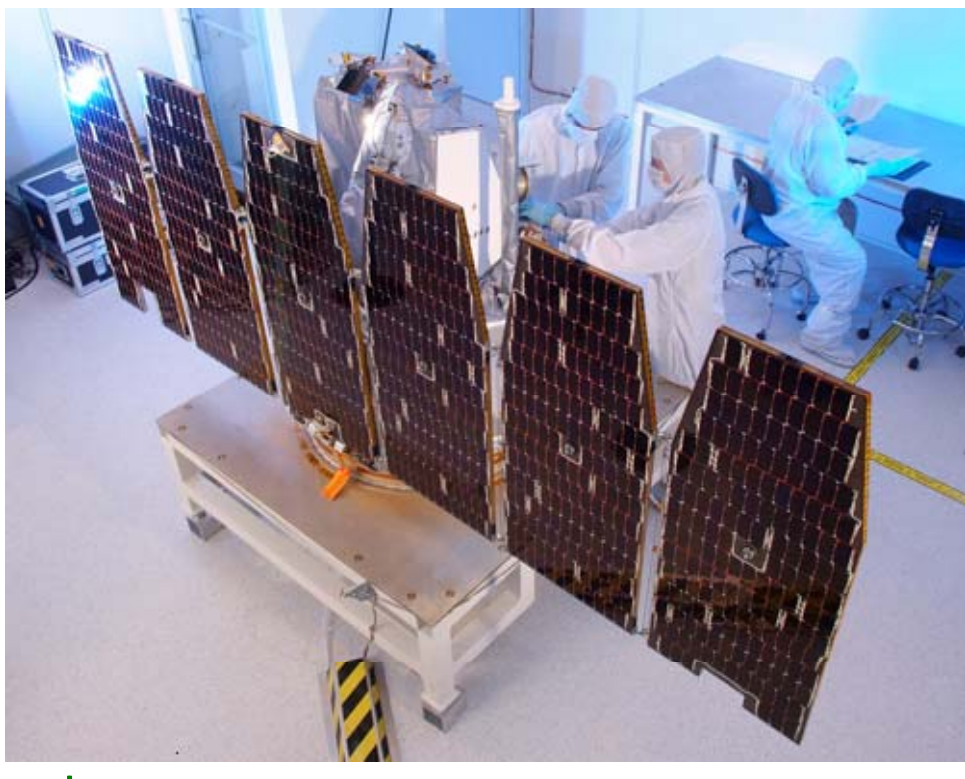

Photo Courtesy of NASA

- Typical Tests

- Payload subsystem

- Structural test assembly

- Mechanical assemblies (e.g. antenna arrays)

- Propulsion subsystem 


\section{Subsystem Qualification}

\begin{tabular}{|c|c|c|c|c|c|c|c|}
\hline Test & Reference & $\begin{array}{c}\text { Suggested } \\
\text { Sequence }\end{array}$ & $\begin{array}{l}\text { Payload } \\
\text { Fairing }\end{array}$ & Structure & Bus & $\begin{array}{l}\text { Payload / } \\
\text { Instrument }\end{array}$ & $\begin{array}{c}\text { Multi-Unit } \\
\text { Module }\end{array}$ \\
\hline Inspection & 4.6 & 1,11 & $\mathrm{R}$ & $\mathrm{R}$ & $\mathrm{R}$ & $\mathrm{R}$ & $\mathrm{R}$ \\
\hline $\begin{array}{l}\text { Specification } \\
\text { Performance } \\
(1)\end{array}$ & 7.3 .1 & 2,10 & $\mathrm{R}$ & $\mathrm{R}$ & $\mathrm{R}$ & $\mathrm{R}$ & $\mathrm{R}$ \\
\hline Static Load & 7.3 .2 & 3 & $\mathrm{R}$ & $\mathrm{R}$ & $\mathrm{R}$ & $\mathrm{R}$ & $\mathrm{R}$ \\
\hline Pressure and Leak & 7.3 .3 & 4 & ER & ER & $\mathrm{ER}^{(4)}$ & ER & ER \\
\hline Shock & 7.3 .6 & 7 & - & ER & ER & $\mathrm{ER}$ & ER \\
\hline $\begin{array}{l}\text { Random Vibration } \\
\text { or } \\
\text { Acoustic }\end{array}$ & $\begin{array}{l}7.3 .4 \\
7.3 .5\end{array}$ & 5 & $\mathrm{R}$ & ER & ER & ER & ER \\
\hline Thermal Vacuum & 7.3.7 & 6 & - & ER & ER & $\mathrm{R}$ & $\mathrm{R}$ \\
\hline $\begin{array}{l}\text { Separation and } \\
\text { Deployment (3) }\end{array}$ & 7.3 .8 & 8 & $\mathrm{R}$ & $\mathrm{R}$ & $\mathrm{R}$ & ER & ER \\
\hline EMC & 7.3 .9 & 9 & - & - & $\mathrm{R}$ & $\mathrm{R}$ & ER \\
\hline Mode Survey & 7.3 .10 & Any & $\mathrm{R}$ & - & $\mathbf{R}^{(2)}$ & $\mathbf{R}^{(2)}$ & ER \\
\hline
\end{tabular}

$\mathrm{R} \quad$ Required

ER Evaluation Required (See 6.3)

(1) Specification performance tests shall be conducted prior to, during and following each environmental test.,as appropriate

(2) Mode survey testing is required for both the either at the Subsystem or the System level.

(3) First motion testing of separable/deployable components required. 


\section{Subsystem Acceptance}

\begin{tabular}{|c|c|c|c|c|c|c|c|}
\hline Test & Reference & $\begin{array}{l}\text { Suggested } \\
\text { Sequence }\end{array}$ & $\begin{array}{c}\text { Payload } \\
\text { Fairing }\end{array}$ & Structure & Bus & Payload & $\begin{array}{c}\text { Multi-Unit } \\
\text { Module }\end{array}$ \\
\hline Inspection & 4.6 & 1,10 & $\mathrm{R}$ & $\mathrm{R}$ & $\mathrm{R}$ & $\mathrm{R}$ & $\mathrm{R}$ \\
\hline $\begin{array}{l}\text { Specification } \\
\text { Performance }^{(1)}\end{array}$ & 7.3 .1 & 2,9 & $\mathrm{R}$ & $\mathrm{R}$ & $\mathrm{R}$ & $\mathrm{R}$ & $\mathrm{R}$ \\
\hline Static Load & 7.3 .2 & 3 & $\mathbf{R}^{(2)}$ & $\mathbf{R}^{(2)}$ & $\mathbf{R}^{(2)}$ & $\mathrm{R}$ & - \\
\hline Pressure and Leak & 7.3 .3 & 4 & - & - & $\mathrm{R}$ & $\mathrm{R}$ & $\mathrm{R}$ \\
\hline $\begin{array}{l}\text { Random Vibration } \\
\text { or } \\
\text { Acoustic }\end{array}$ & $\begin{array}{l}7.3 .4 \\
7.3 .5\end{array}$ & 5 & ER & - & - & ER & $\mathrm{R}$ \\
\hline Thermal Vacuum & 7.3 .7 & 6 & - & - & ER & $\mathrm{R}$ & $\mathrm{R}$ \\
\hline $\begin{array}{l}\text { Separation and } \\
\text { Deployment }{ }^{(3)}\end{array}$ & 7.3 .8 & 7 & ER & - & ER & ER & ER \\
\hline $\mathrm{EMC}^{(4)}$ & 7.3 .9 & 8 & - & - & ER & ER & - \\
\hline
\end{tabular}

$\mathrm{R} \quad$ Required

ER Evaluation Required (See 6.3)

(1) Specification performance tests shall be conducted prior to, during and following each environmental test., as appropriate

(2) Required for composite and bonded structures. Evaluation required for all other structures.

(3) First motion testing of separable/deployable components required.

(4) Required when there is less than $12 \mathrm{~dB}$ margin. 


\section{Subsystem Level Margins and Durations}

\begin{tabular}{|c|c|c|c|}
\hline Test & Qualification & Protoqualification & Acceptance \\
\hline Shock & $\begin{array}{l}1 \text { activation of all shock- } \\
\text { producing events; } \\
2 \text { additional activation of } \\
\text { significant events }\end{array}$ & $\begin{array}{l}1 \text { activation of all shock- } \\
\text { producing events; } \\
1 \text { additional activation of } \\
\text { significant events }\end{array}$ & $\begin{array}{l}1 \text { activation of significant } \\
\text { shock-producing events }\end{array}$ \\
\hline Acoustic $^{(1)}$ & $\begin{array}{l}6 \mathrm{~dB} \text { above acceptance for } \\
3 \text { minutes }\end{array}$ & $\begin{array}{l}3 \mathrm{~dB} \text { above acceptance for } \\
2 \text { minutes }\end{array}$ & $\begin{array}{l}\text { Envelope of MPE and } \\
\text { minimum spectrum } \\
\text { (Figure 6.3.6-1) for } 1 \text { minute }\end{array}$ \\
\hline Vibration $^{(1)}$ & $\begin{array}{l}6 \mathrm{~dB} \text { above acceptance for } \\
3 \text { minutes in each of } \\
3 \text { axes }\end{array}$ & $\begin{array}{l}3 \mathrm{~dB} \text { above acceptance for } \\
2 \text { minutes in each of } \\
3 \text { axes }\end{array}$ & $\begin{array}{l}\text { Envelope of MPE and } \\
\text { minimum spectrum } \\
\text { (Figure } 8.3 .7-1 \text { ) for } 1 \text { minute } \\
\text { in each of } 3 \text { axes }\end{array}$ \\
\hline $\begin{array}{l}\text { Thermal } \\
\text { Vacuum }\end{array}$ & $\begin{array}{l} \pm 10^{\circ} \mathrm{C} \text { beyond acceptance } \\
\text { for } 8 \text { cycles }\end{array}$ & $\begin{array}{l} \pm 5^{\circ} \mathrm{C} \text { beyond acceptance } \\
\text { for } 4 \text { cycles }\end{array}$ & MPT for 4 cycles \\
\hline $\begin{array}{l}\text { Static } \\
\text { Load(2) }^{(2)}\end{array}$ & $\begin{array}{l}1.25 \text { times the limit load } \\
\text { for unmanned flight or } \\
1.4 \text { times limit load for } \\
\text { manned flight, duration } \\
\text { sufficient to record data }\end{array}$ & $\begin{array}{l}1.25 \text { times the limit load } \\
\text { for unmanned flight or } \\
1.25 \text { times limit load for } \\
\text { manned flight, duration } \\
\text { sufficient to record data }\end{array}$ & $\begin{array}{l}1.1 \text { times the limit load for } \\
\text { bonded, composite, or } \\
\text { sandwich structures, duration } \\
\text { sufficient to record data }\end{array}$ \\
\hline $\begin{array}{l}\text { Pressure } \\
\text { and Leak }\end{array}$ & $\begin{array}{l}1.1 \text { times MEOP for } \\
\text { pressurized structures; } \\
1.25 \text { times MEOP for } \\
\text { pressure vessels; } 1.5 \text { times } \\
\text { MEOP for pressure } \\
\text { components. Pressure } \\
\text { testing followed byleak } \\
\text { testing at MEOP. Other } \\
\text { metallic pressurized } \\
\text { hardware items per } \\
\text { AIAA S-080 and } \\
\text { S-081 }\end{array}$ & $\begin{array}{l}1.1 \text { times MEOP for } \\
\text { pressurized structures; } \\
1.25 \text { times MEOP for } \\
\text { pressure vessels; } 1.5 \text { times } \\
\text { MEOP for pressure } \\
\text { components. Pressure } \\
\text { testing followed byleak } \\
\text { testing at MEOP. Other } \\
\text { metallic pressurized } \\
\text { hardware items per } \\
\text { AIAA S-080 and } \\
\text { S-081 }\end{array}$ & $\begin{array}{l}1.1 \text { times MEOP for } \\
\text { pressurized structures; } 1.25 \\
\text { times MEOP for pressure } \\
\text { vessels; } 1.5 \text { times MEOP for } \\
\text { pressure components. } \\
\text { Pressure testing followed by } \\
\text { leak testing at MEOP. Other } \\
\text { metallic pressurized hardware } \\
\text { items per AIAA S-080 } \\
\text { and S-081 }\end{array}$ \\
\hline EMC & $\begin{array}{l}12 \mathrm{~dB} \text { minimum, duration } \\
\text { same as acceptance }\end{array}$ & $\begin{array}{l}6 \mathrm{~dB} \text { minimum, duration } \\
\text { same as acceptance }\end{array}$ & $\begin{array}{l}6 \mathrm{~dB} \text { minimum, } 20 \text { minutes at } \\
\text { each space vehicle transmitter } \\
\text { frequency for radiated } \\
\text { susceptibility }\end{array}$ \\
\hline
\end{tabular}

(1) See 10.2.3 for subsystems with effective duration greater than 15 seconds.

(2) Refer to AIAA S-1 10-2005 and TOR-2003(8583)-2894

(3) Refer to TOR-2003(8584)-2895, SMC-S-005, and SMC-S-006 


\section{Test Objectives - System}

- Definition

- Composite of equipment, skills, and techniques capable of performing or supporting an operational role

- Includes all operational equipment, related facilities, material, software, services, and personnel required for its operation

- Test Objective

- Verify interfaces and key performance parameters

- Validate operational capabilities

- Validate specification requirements

- Functional and performance tests

- Will have flight software and firmware

- Environmental tests

\section{Usually the $1^{\text {st }}$ Time It All Comes Together}

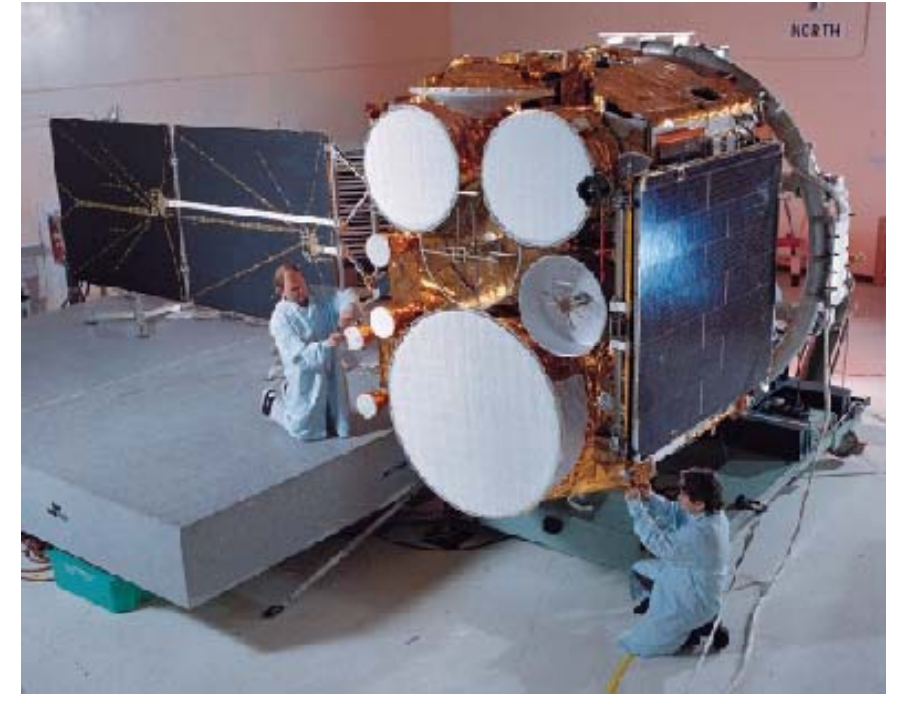

Courtesy of Lockheed Martin 


\section{Preferred System Test Sequence}

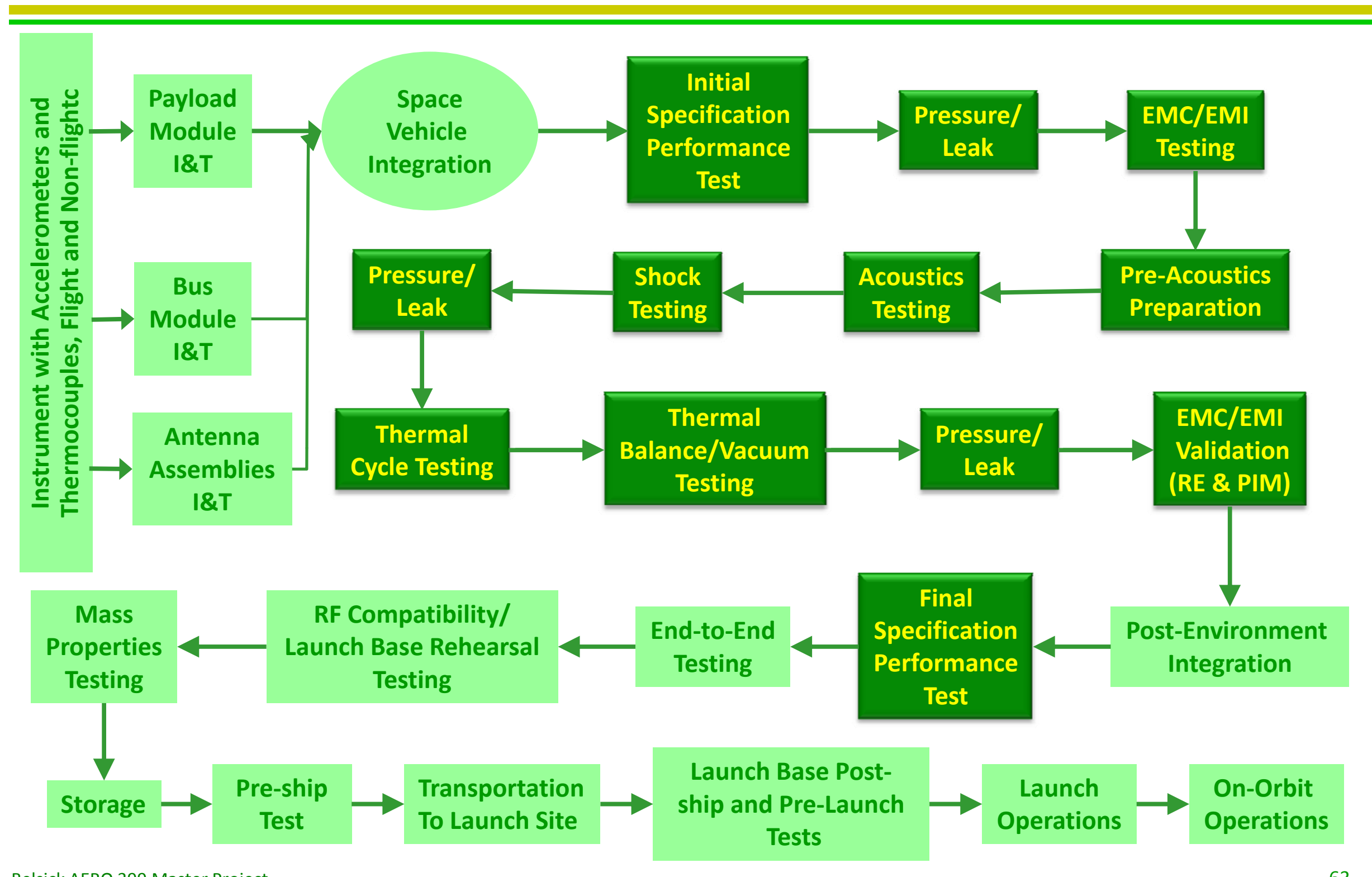




\section{System Qualification}

\begin{tabular}{||l|c|c|c|c|c||}
\hline \hline \multicolumn{1}{|c|}{ Test } & Section & $\begin{array}{c}\text { Suggested } \\
\text { Sequence }\end{array}$ & $\begin{array}{c}\text { Launch } \\
\text { Vehicle }\end{array}$ & $\begin{array}{c}\text { Upper-stage } \\
\text { Vehicle }\end{array}$ & $\begin{array}{c}\text { Space } \\
\text { Vehicle }\end{array}$ \\
\hline Inspection & 4.6 & 1,13 & $\mathrm{R}$ & $\mathrm{R}$ & $\mathrm{R}$ \\
\hline $\begin{array}{l}\text { Specification } \\
\text { Performance }\end{array}$ & 8.3 .1 & 2,12 & $\mathrm{R}$ & $\mathrm{R}$ & $\mathrm{R}$ \\
\begin{tabular}{|l} 
Pressure/Leakage \\
EMC
\end{tabular} & 8.3 .2 & $3,7,10$ & $\mathrm{R}$ & $\mathrm{R}$ & $\mathrm{R}$ \\
\hline $\begin{array}{l}\text { Shock } \\
\begin{array}{l}\text { Acoustic } \\
\text { or } \\
\text { Random Vibration }\end{array}\end{array}$ & 8.3 .3 & 4 or $11^{(2)}$ & $\mathrm{R}$ & $\mathrm{R}$ & $\mathrm{R}$ \\
$\begin{array}{l}\text { Thermal Balance } \\
\text { Thermal Vacuum }\end{array}$ & 8.3 .6 & 6 & $\mathrm{R}$ & $\mathrm{R}$ & $\mathrm{R}$ \\
\hline Mode Survey & 8.3 .7 & 8 & -- & $\mathrm{ER}$ & $\mathrm{R}$ \\
\hline
\end{tabular}

\section{R Required}

ER Evaluation Required

(1) Electrical and mechanical specification performance tests shall be conducted prior to, during and following each environmental test, as appropriate.

(2) EMC testing, sequence 4 or 11 , shall be conducted when there are radiated emission requirements

below $10 \mathrm{dBuV} / \mathrm{m}$ or there is a requirement on passive intermodulation levels.

(3) Vibration can be used in place of acoustics for vehicle weights under 180 kilograms. 


\section{System Acceptance}

\begin{tabular}{|c|c|c|c|c|c|}
\hline Test & $\begin{array}{l}\text { Reference } \\
\text { Paragraph }\end{array}$ & $\begin{array}{l}\text { Suggested } \\
\text { Sequence }\end{array}$ & $\begin{array}{l}\text { Launch } \\
\text { Vehicle }\end{array}$ & $\begin{array}{l}\text { Upper-stage } \\
\text { Vehicle }\end{array}$ & $\begin{array}{l}\text { Space } \\
\text { Vehicle }\end{array}$ \\
\hline Inspection & 4.6 & 1,12 & $\mathrm{R}$ & $\mathrm{R}$ & $\mathrm{R}$ \\
\hline $\begin{array}{l}\text { Specification } \\
\text { Performance }^{(1)}\end{array}$ & 8.3 .1 & 2,11 & $\mathrm{R}$ & $\mathrm{R}$ & $\mathrm{R}$ \\
\hline Pressure/Leak & 8.3 .2 & $3,7,9$ & $\mathrm{R}$ & $\mathrm{R}$ & $\mathrm{R}$ \\
\hline $\mathrm{EMC}^{(2)}$ & 8.3 .3 & 4 or 10 & ER & ER & ER \\
\hline Shock & 8.3 .4 & 6 & ER & ER & $\mathrm{R}$ \\
\hline $\begin{array}{l}\text { Acoustic } \\
\text { or } \\
\text { Vibration }{ }^{(3)}\end{array}$ & $\begin{array}{l}8.3 .5 \\
8.3 .6\end{array}$ & 5 & ER & $\mathrm{R}$ & $\mathrm{R}$ \\
\hline Thermal Vacuum & 8.3 .8 & 8 & -- & $\mathrm{R}$ & $\mathrm{R}$ \\
\hline
\end{tabular}

\section{R Required}

ER Evaluation Required

(1) Electrical and mechanical specification performance tests shall be conducted prior to, during and following each environmental test as appropriate.

(2) EMC testing (sequence 4 and 10) required when there is less than $12 \mathrm{~dB}$ margin.

(3) Vibration can be used in lieu of acoustics for vehicles under $180 \mathrm{~kg}$. 


\section{System Level Margins and Durations}

\begin{tabular}{|c|c|c|c|}
\hline Test & Qualification & Protoqualification & Acceptance \\
\hline Shock & $\begin{array}{l}1 \text { activation of all shock- } \\
\text { producing events; } 2 \text { additional } \\
\text { activation of significant events }\end{array}$ & $\begin{array}{l}1 \text { activation of all shock- } \\
\text { producing events; } 1 \text { additional } \\
\text { activation of significant events }\end{array}$ & $\begin{array}{l}1 \text { activation of significant } \\
\text { shock-producing events }\end{array}$ \\
\hline Acoustic $^{(1)}$ & $\begin{array}{l}6 \mathrm{~dB} \text { above acceptance for } \\
3 \text { minutes }\end{array}$ & $\begin{array}{l}3 \mathrm{~dB} \text { above acceptance for } \\
2 \text { minutes }\end{array}$ & $\begin{array}{l}\text { Envelope of MPE and } \\
\text { minimum spectrum } \\
\text { (Figure 6.3.6-1) for } 1 \text { minute }\end{array}$ \\
\hline Vibration $^{(1)}$ & $\begin{array}{l}6 \mathrm{~dB} \text { above acceptance for } \\
3 \text { minutes in each of } 3 \text { axes }\end{array}$ & $\begin{array}{l}3 \mathrm{~dB} \text { above acceptance for } \\
2 \text { minutes in each of } 3 \text { axes }\end{array}$ & $\begin{array}{l}\text { Envelope of MPE and } \\
\text { minimum spectrum } \\
\text { (Figure } 8.3 .7-1 \text { ) for } 1 \text { minute } \\
\text { in each of } 3 \text { axes }\end{array}$ \\
\hline $\begin{array}{l}\text { Thermal } \\
\text { Vacuum }^{(2)}\end{array}$ & $\begin{array}{l} \pm 10^{\circ} \mathrm{C} \text { beyond acceptance for } \\
8 \text { cycles }\end{array}$ & $\begin{array}{l} \pm 5^{\circ} \mathrm{C} \text { beyond acceptance for } \\
4 \text { cycles }\end{array}$ & MPT for 4 cycles \\
\hline $\begin{array}{l}\text { Proof } \\
\text { Pressure }\end{array}$ & $\begin{array}{l}1.1 \text { times MEOP for } \\
\text { pressurized structures; } 1.25 \\
\text { times MEOP for pressure } \\
\text { vessels; } 1.5 \text { times MEOP for } \\
\text { pressure components. Other } \\
\text { metallic pressurized hardware } \\
\text { items per AIAA S-080 } \\
\text { and S-081. }\end{array}$ & $\begin{array}{l}1.1 \text { times MEOP for } \\
\text { pressurized structures; } 1.25 \\
\text { times MEOP for pressure } \\
\text { vessels; } 1.5 \text { times MEOP for } \\
\text { pressure components. Other } \\
\text { metallic pressurized hardware } \\
\text { items per AIAA S-080 } \\
\text { and S-081. }\end{array}$ & $\begin{array}{l}1.1 \text { times MEOP for } \\
\text { pressurized structures; } 1.25 \\
\text { times MEOP for pressure } \\
\text { vessels; } 1.5 \text { times MEOP for } \\
\text { pressure components. Other } \\
\text { metallic pressurized hardware } \\
\text { items per AIAA S-080 } \\
\text { and S-081. }\end{array}$ \\
\hline EMC & $\begin{array}{l}12 \mathrm{~dB} \text { minimum, duration } \\
\text { same as acceptance }\end{array}$ & $\begin{array}{l}6 \mathrm{~dB} \text { minimum, duration } \\
\text { same as acceptance }\end{array}$ & $\begin{array}{l}6 \mathrm{~dB} \text { minimum, } 20 \text { minutes at } \\
\text { each space vehicle transmitter } \\
\text { frequency for radiated } \\
\text { susceptibility }\end{array}$ \\
\hline
\end{tabular}

(2) See 10.2.3 for units with effective duration greater than 15 seconds

(2) See Note 10.1.1 if vehicle thermal cycle testing is performed.

Ref: TR-2004(8583)-1 Rev A (MIL-STD 1540E) Table 8.3-3 


\section{Test Objectives - System End-to-End}

\section{- Test Objective}

- Verify space vehicle and ground intersegment interface

- Should include space vehicle flight and operations qualified software

- Validate telemetry processing, command capability, and communication between the space vehicle through the ground receiving network to mission processing

- Functional uplink, downlink

- Timing

- Communication

- Launch rehearsal to validate procedures and training

- Typically last electrical test at the launch site

- Common issues

- Antenna polarity

- Crypto key mismatch
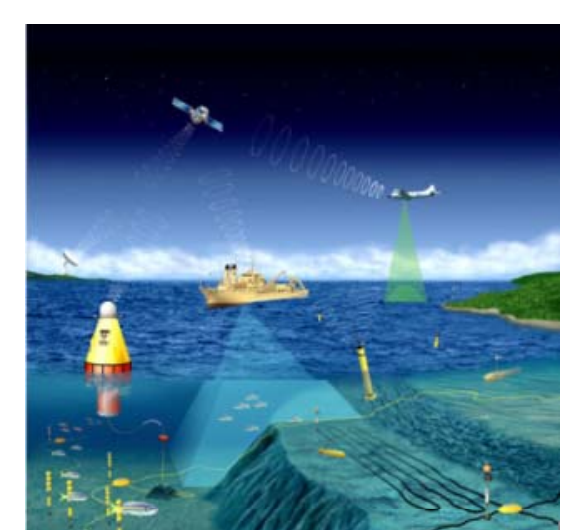

Courtesy of Integrated Ocean Observing System

- Network timing and protocol

\section{Simple Test that Avoids Potential Fatality}




\section{Test Objectives - Pre-Launch}

- Demonstrate readiness of the hardware, software, personnel, procedures, and mission interfaces to support launch and the program mission

- Assure readiness for space vehicle integration with the launch vehicle and launch facility

- Verify that no changes have occurred in vehicle parameters as a result of handling and transportation to the launch base

- Demonstrate interface compatibility

- Assure readiness to launch

- Demonstrate successful integration of the launch and space vehicles with the launch facility

- Demonstrate compatibility exists between the integrated vehicle hardware and software, ground equipment and software, and within the entire launch and on-orbit system (end-to-end)

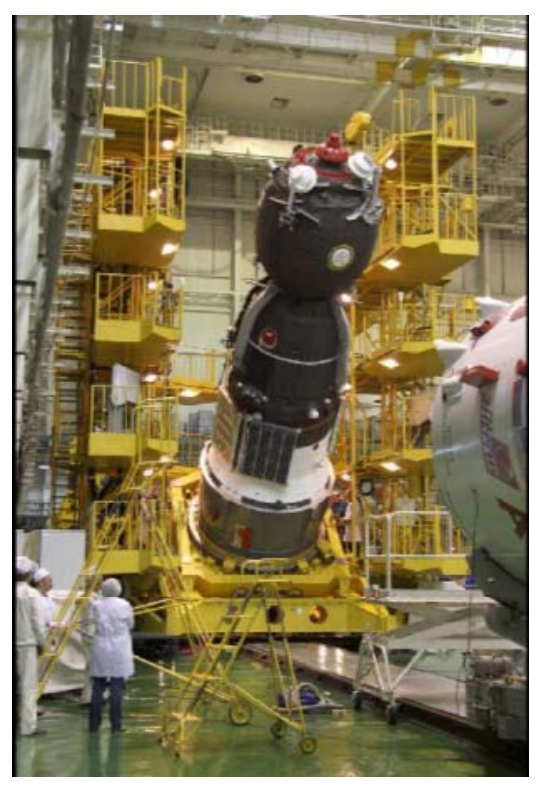

Courtesy of NASA

\section{Perform Before and After Shipment to Launch Site}




\section{Typical Pre-Launch Flow}

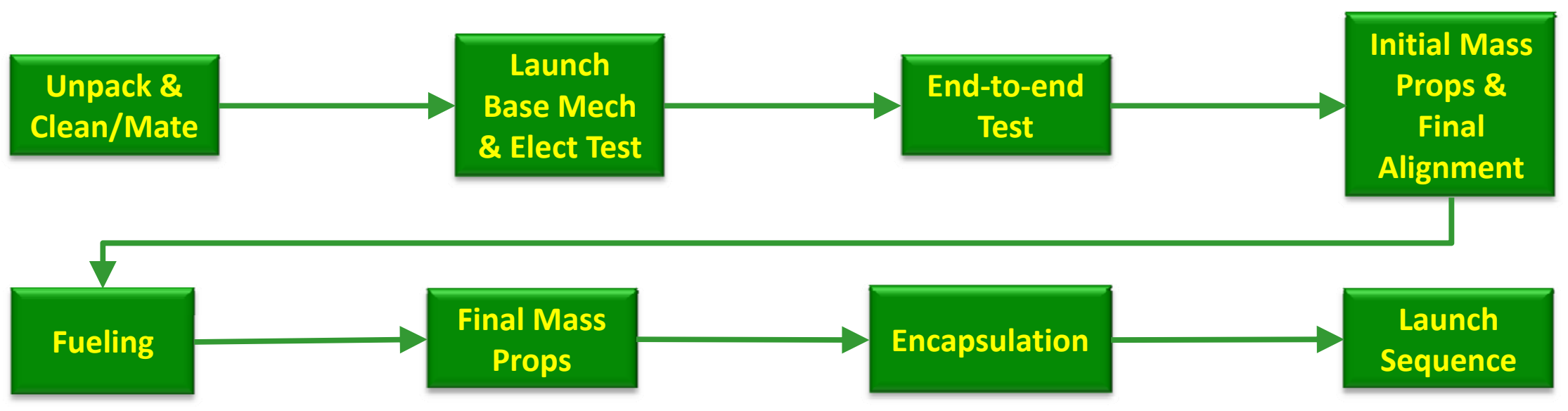

- Precursors to launch processing

- Launch vehicle dynamics analysis

- LV adapter fitcheck and deployment

- LV electrical interface checkout

- Mass properties

- Mechanical fit checks and alignments
- Electrical tests

- In stowed position

- Ideally end to end using antenna feed couplers, otherwise hardline

- End to end functionality and launch sequence check (all copper paths)

Make Sure You Leave the Vehicle in its Final Launch Configuration!! 


\section{Test Objectives - On-Orbit}

- To initialize and verify functional integrity of the space vehicle following launch

- Demonstrates pace vehicle operating as designed

- Demonstrates ground systems are ready to support mission operations

- Demonstrates Mmssion data can be distributed to the planned users

- Three major phases

- Initialization and checkout

- Operations and maintenance during normal operations

- Anomaly response

- Continuing debate

- Checkout only one string or also redundancy string?

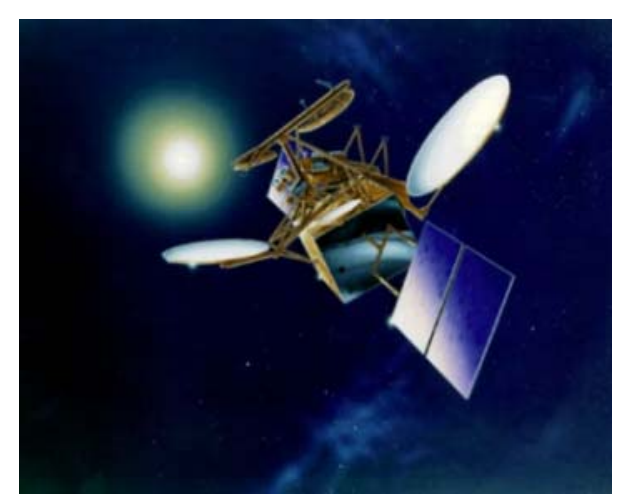

Courtesy of NASA

Must Demonstrate Patch Capability Prior to Launch Just in Case 


\section{Typical On-Orbit Testing Flow}

- Lasts anywhere from 30 days to 2 years

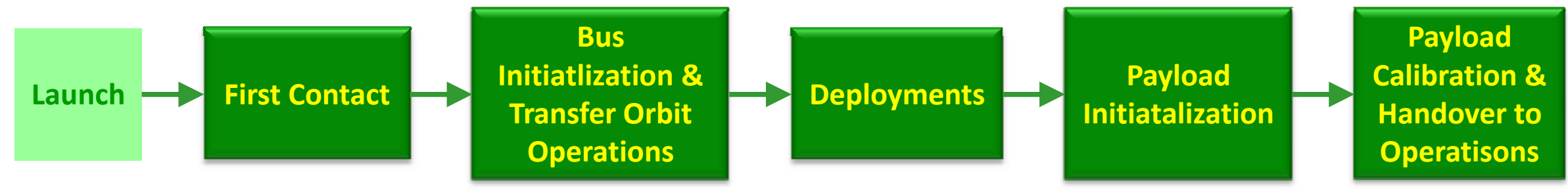

L+ 5 Days* Installation Functional Checks Calibration
$L+60$ Days* Satellite Subsystem/Payload Characterization Interface and Performance Validation Further calibration and Database Updates

Lission Scenarios
Effectvienss Suitability
Mission Certification

*Timelines vary for each program according to complexity and problems encountered after launch 


\section{Test Descriptions}




\section{Test Configuration Issues}

- What flight products are not included?

- What non-flight products are included?

- How will you handle missing hardware and software?

- Mass model? Engineering unit? Simulator?

- What functional performance parameters will be monitored during a test?

- How will you monitor the performance parameters?

- Telemetry? Is sample rate adequate?

- Hardwire?

- When are abbreviated functional tests acceptable?

- Which functional parameters constitute an abbreviated functional test?

- Will tanks be loaded?

- With what? What about cryogenic fluids?

- Will operating pressures be used?

\section{Understand and document test configuration}




\section{Wear-in/Burn-in}

Purpose: Propagate latent infant mortality defects and to assure smooth and consistent performance

Wear-in

- Test Requirements

- Number of cycles: 15 or $5 \%$ of the total number of expected cycles during service life

- Post environment test

- Test Challenges

- Perform prior to other tests

- Configuration

- Applies to moving mechanical assemblies and propulsion devices (e.g..., thrusters and valves)
Burn-in

- Test Requirements

- Minimum 200 hours powered-on at high temeprature

- Last 100 hours error free

- Test challenges

- Perform during thermal testing may need to allow for the additional hours

- Configuration

- Applies to electronic assemblies

Fail Criteria: Does not perform as expected 


\section{Performance Test}

- Purpose

- Verify electrical, optical, and mechanical performance with/without applied environments

- Test Requirements

- Voltages, impedances, frequencies, pulses, waveforms,

- Alignment, pressure, torque angle damping, etc

- Configuration

- Bread board

- Brass board

- Flight quality

- Test Challenges

- Breakout boxes

- Telemetry rate (perceptiveness)

- Simulators for companion signals

- Fail Criteria

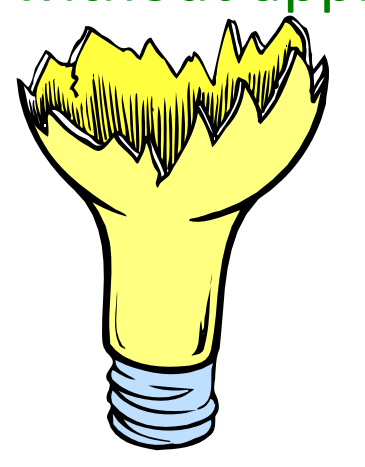

IT FAILED!

- Outside specified performance parameters

\section{It's Performance, Not Functional Test!}

What about the case where performance is off nominal but within limits? 


\section{Specification Performance Test (Subsystem and System)}

- Verifies/validates mechanical and electrical performance of vehicle

- Uitlizes flight products including software

- Includes mechanical deployments

- Performed before and after environmental exposures

- Abbreviated test often performed between environments for risk mitigation

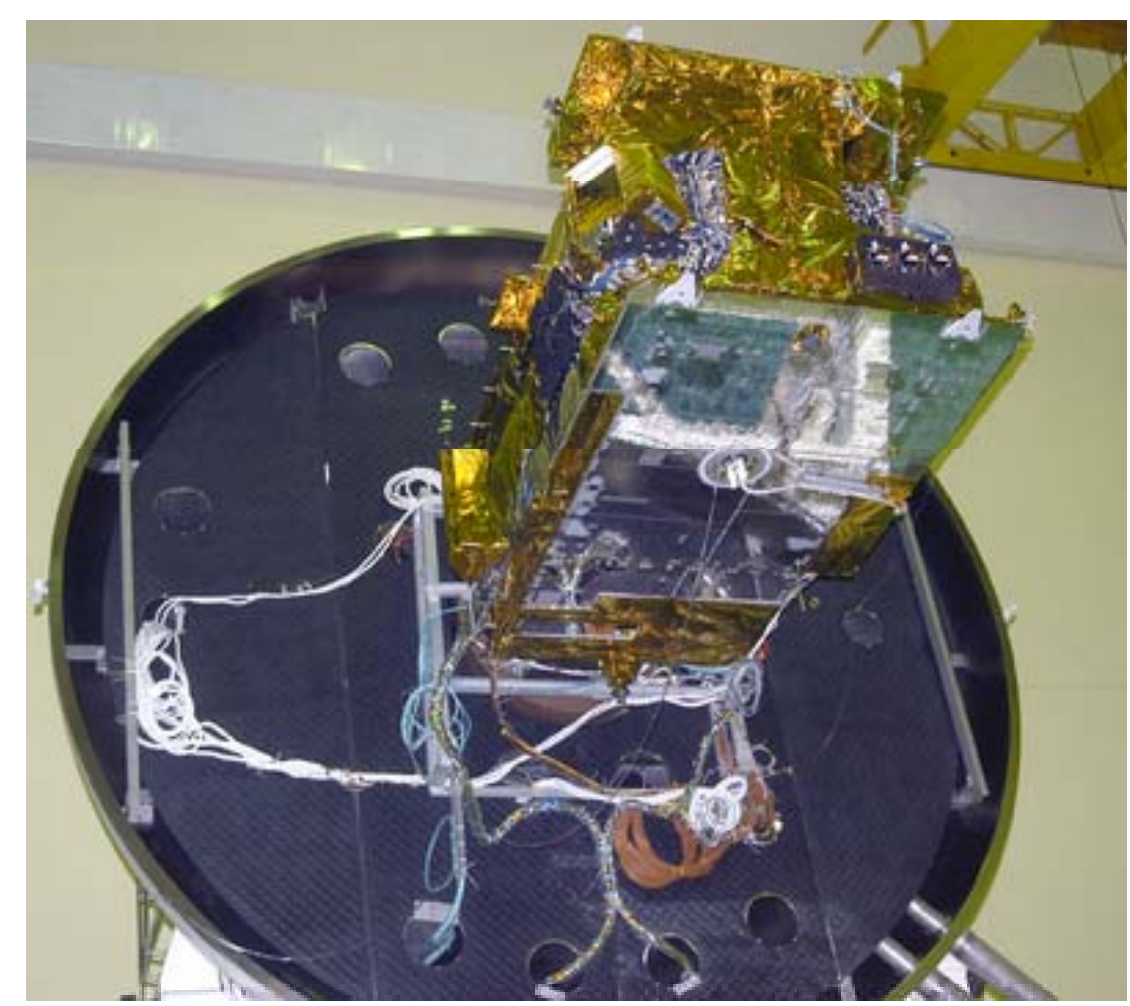

Photo courtesy of ESA

\section{Initial Performance Test Finds, the Greatest Number of Defects (\& Escapes)}




\section{Hardware Environmental Exposure}

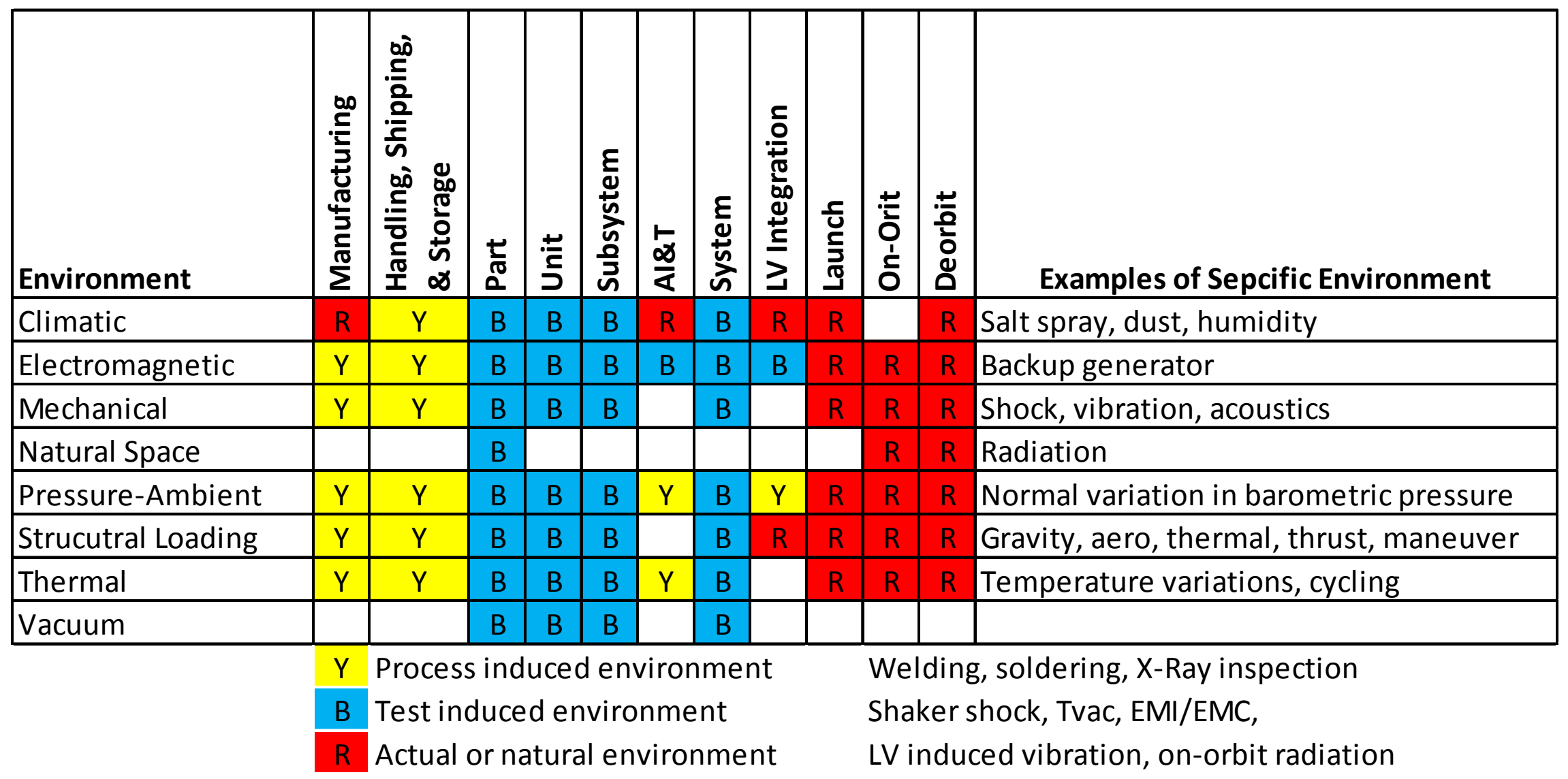




\section{Dynamic Test}

- Purpose

- Demonstrate ability of the system to meet performance requirements during and after exposure to the mission dynamic environment and to demonstrate design margin at qualification

- Test Types

- Vibration: Typically part and unit level

- Acoustic: Typically system level and large structure unit level

- Shock: All levels

- Test Challenges

- Very high test levels, greater than $100 \mathrm{~g}_{\mathrm{rms}}$

- Tests must be split up into frequency bands

- Helpful Hints

- Abbreviated pre- and post-environment tests typically performed

- Prefer to perform powered on and monitored

- Perform before thermal testing

- Done in the order of launch exposure

- Has a higher test effectiveness - will find more failures before launch 


\section{Dynamic Environments During Launch and Ascent}

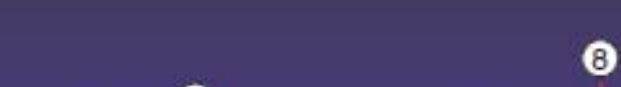

$0_{3}{ }^{4} \quad 6 \quad 6$

8

8

$\ominus$

10

(11)

(12)

(13)

Measured dynamic accelerations

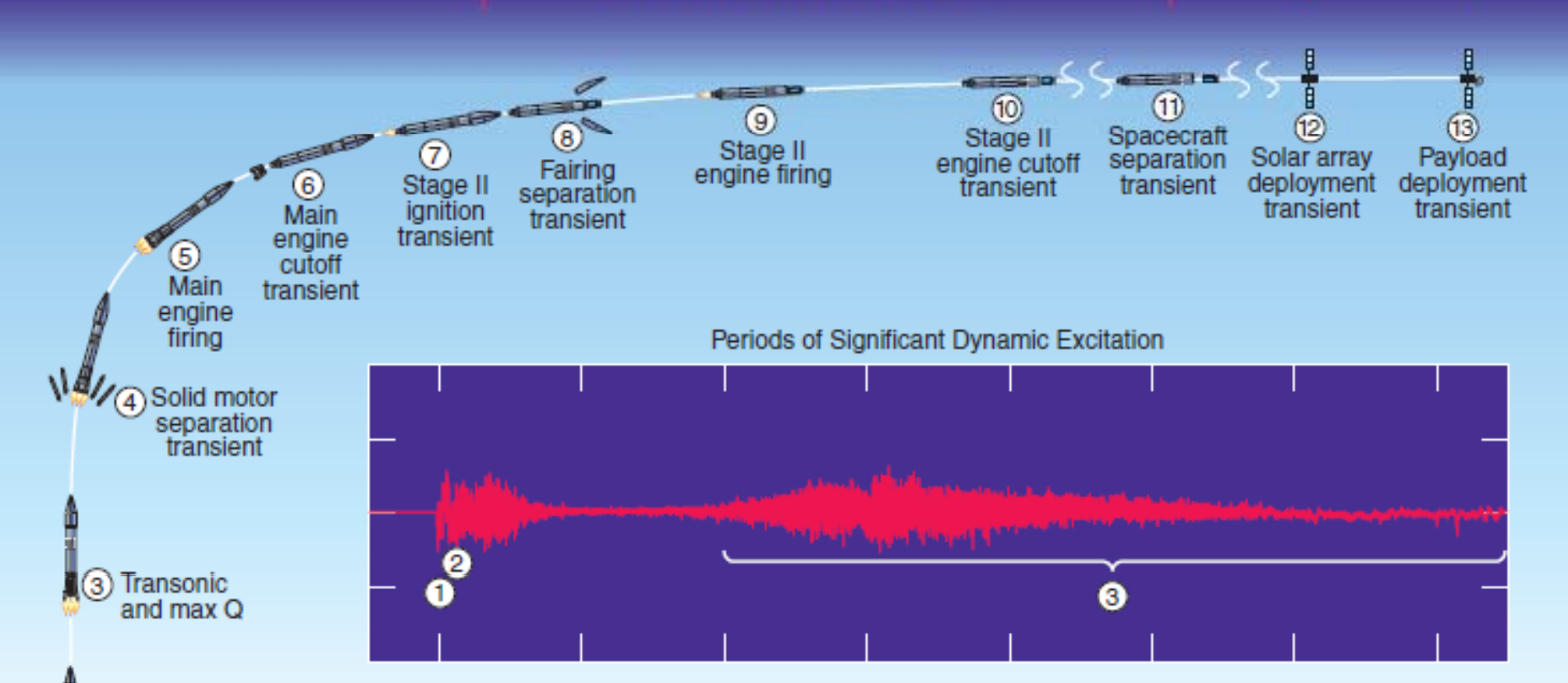

$$
\begin{aligned}
& \text { (2) Liftoff } \\
& \text { (1) Ignition }
\end{aligned}
$$

\section{Ground Processing Environments May Set the Limits}




\section{Dynamic Testing - It's a Big Frequency Range cAlie}

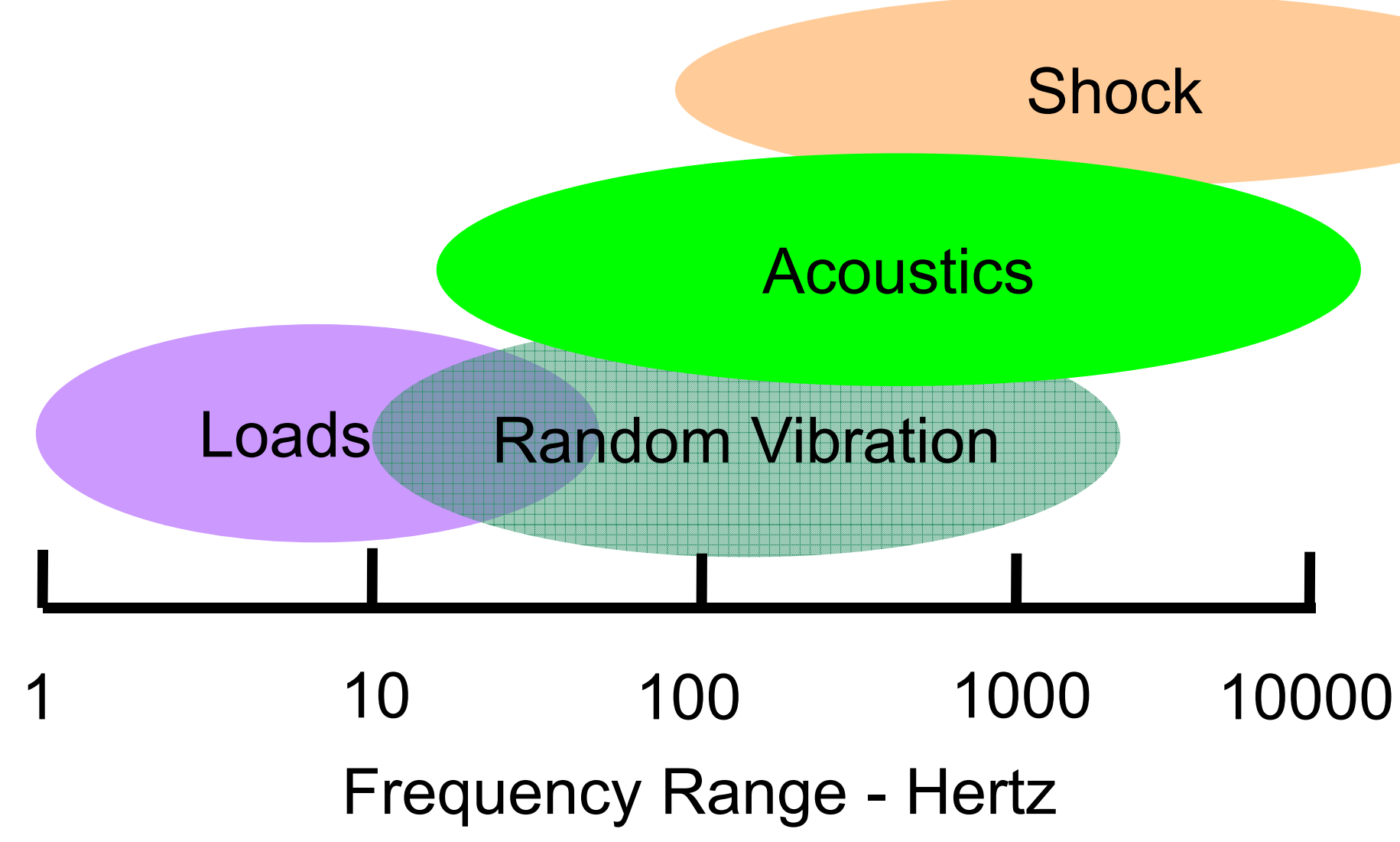

Dynamic Tests Do Not Cover the Same Frequency Range 


\section{Vibration Test}

- Purpose

- Workmanship screening

- Product specification

- Random

- Sine amplitude

- Sine sweep

- Test Requirements

- Test tolerance bands

- Large table with hydraulic actuators (covers $2-100 \mathrm{~Hz}$ )

- Large table with magnetic coil and armature (covers $20-2000 \mathrm{~Hz}$ )

- Configuration

- Unit - Flight, powered, functionally exercised
TWINS Qualification Random Vibration Spectrum

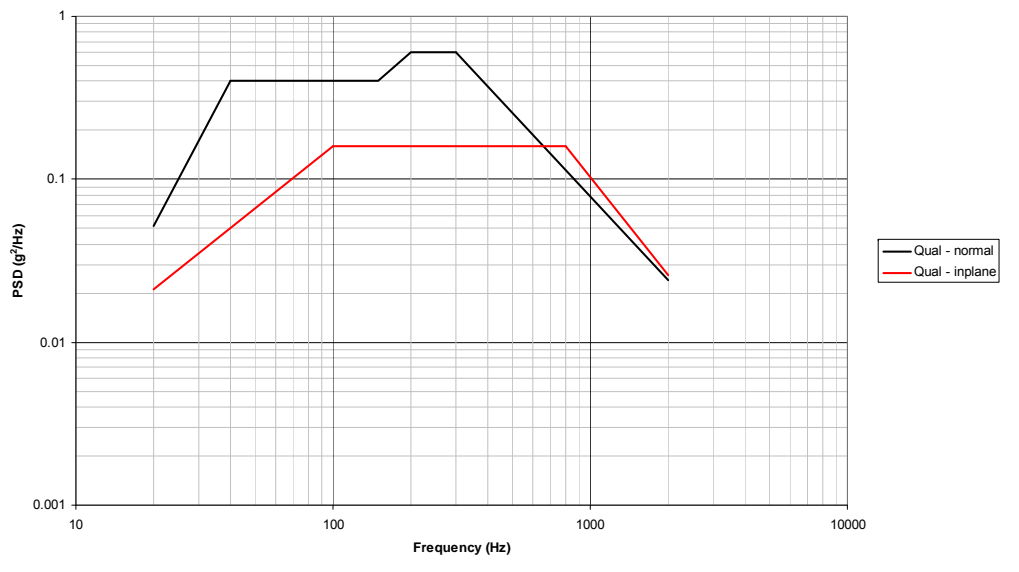

Picture courtesy of The Aerospace Institute

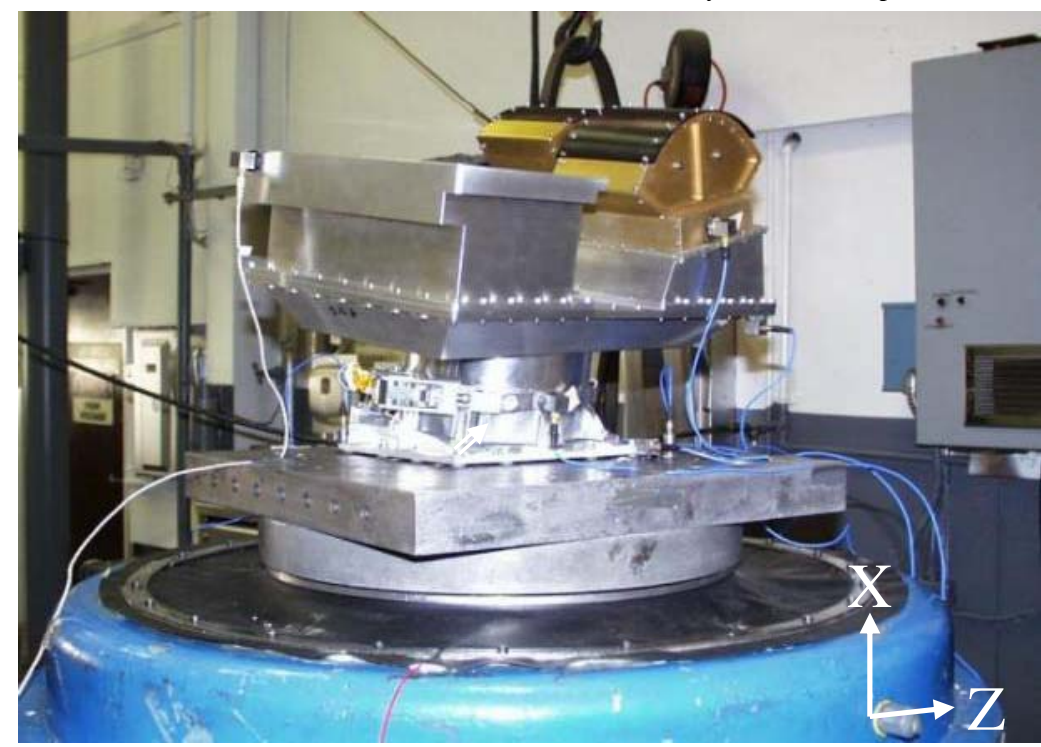




\section{Vibration Test (cont)}

- Test Challenges

- Duration effects fatigue life

- Life demonstrated during qualification

- Which axis first

- Fixture resonance

- Penalty testing

- Fail Criteria

- Fails performance test during or after exposure

- Fails deployment test after exposure

- Structure cracks or yields, torque relaxed

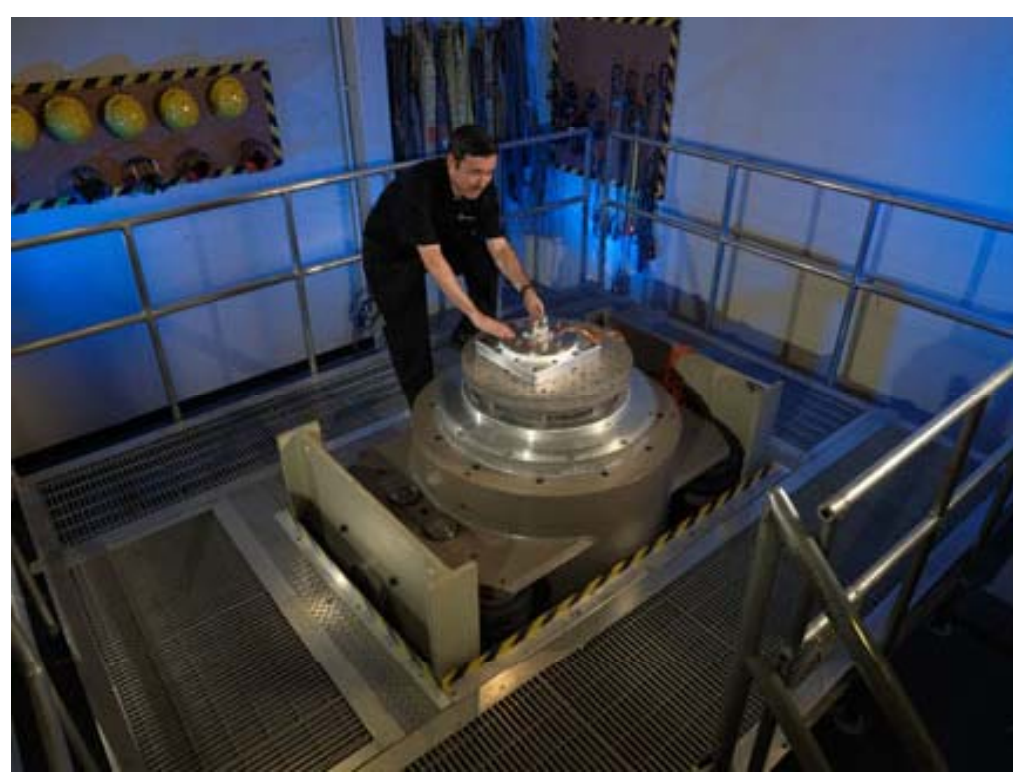

Photo courtesy of NASA

- Parts fall off

- Helpful Hints

- Unit should be powered

- Perform before thermal testing

\section{Power-on Vibration Tests Are Preferred Workmanship Tests}




\section{Acoustic Test}

- Purpose

- Product specification

- Can defer to higher level of assembly

- When risk of failure is low

- Don't defer solar arrays and large antennas

- To ensure pressure loading survival for low mass to area hardware

- Test Requirements

- Reverberant chamber - large concrete box with air or nitrogen horns

- Test Setup and Instrumentation

- Pretest shaping runs

- Microphone locations

- Data quality checks

- Overtest protection

- Configuration

- Launch Configuration (stowed)

- Power-on

- MIL-HDBK-340A requires at least all hardware on during ascent

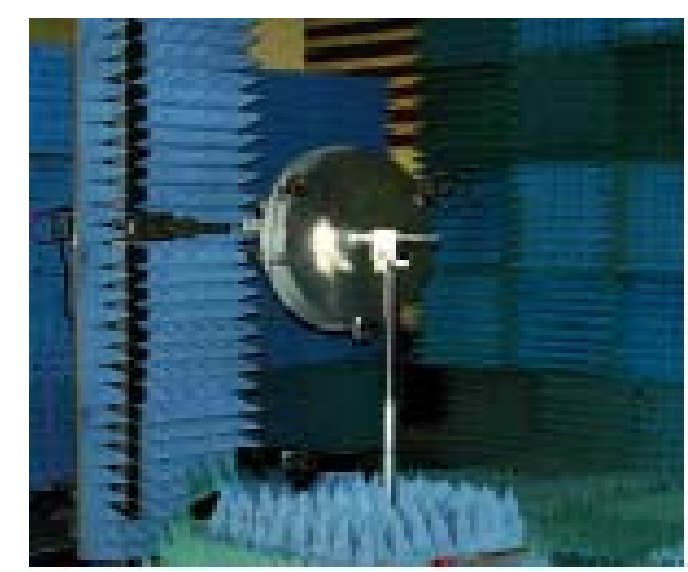

Pictures courtesy of The Aerospace Corp Crosslink@ 


\section{Acoustic Test (cont)}

- Test Challenges

- Spectrum levels and duration, test tolerance bands

- Minimum stress screening level

- Number and placement of control microphones

- Inspection or tests to validate unit passed test

- Pass Criteria

- No power intermittents observed during exposure

- No parts fell on floor

- Structure intact - subsequent deployments nominal

- Subsequent ambient functional or thermal test passed

- Helpful hints

- Typical antenna failures are at supports and laminate debonds

- Typical solar array failures are cracked glass,

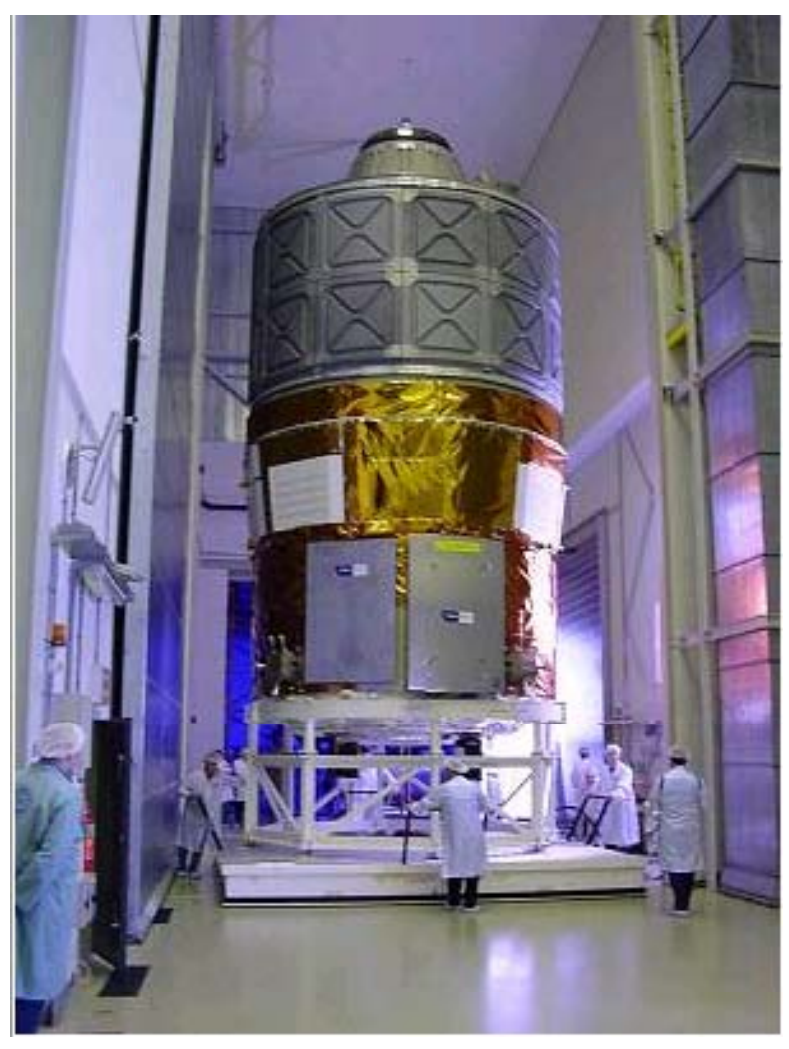

Courtesy of European Space Agency laminate debonds, circuit lifting 


\section{Shock Test}

- Purpose

- Product specification

- Qualification test susceptible units (ex. TWTAs, gyros)

- High frequency events such as deployments and separations for system

- Test Requirements

- Test methods

- Plate (hanging or on table) struck by hammer or ram

- Beam struck by hammer

- Vibration shaker

- Multiple times in each axis

- Amplitude with test tolerance criteria

- Test fixturing used to minimize interference of response spectrum

- Flight representative

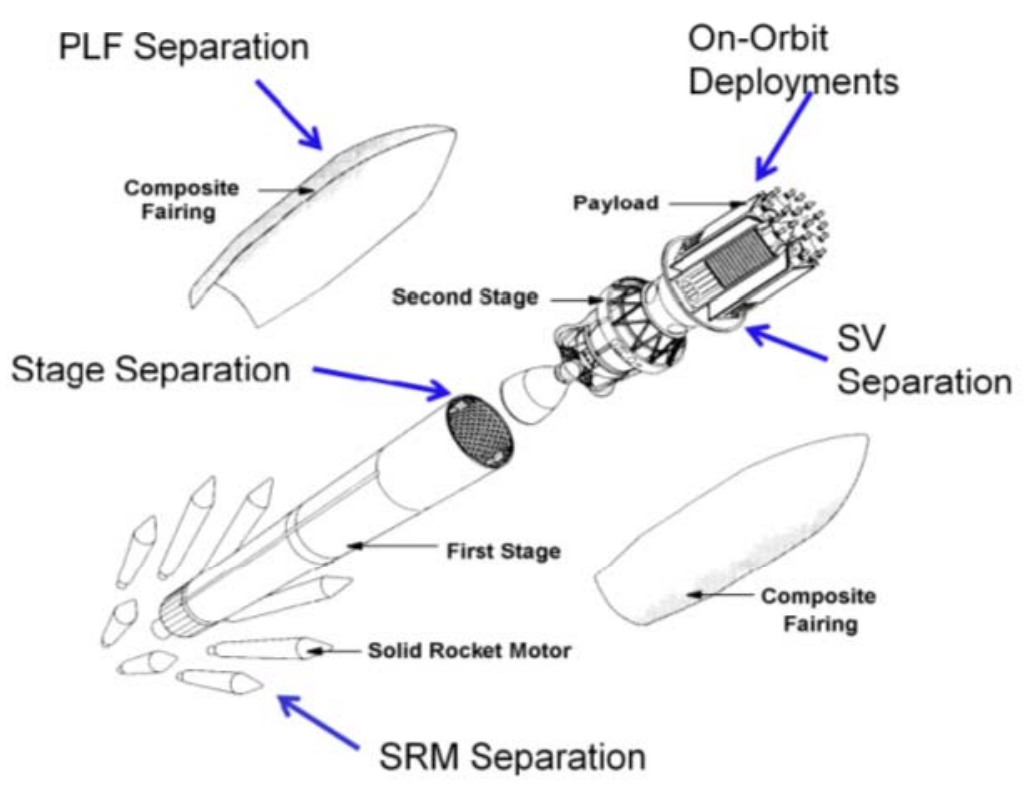




\section{Shock Test (cont)}

- Configuration

- Power on and monitored

- High resolution needed

- Relay chatter detection

- Test Challenges

- Which units are susceptible

- Coverage by random environment

- Test Setup and Instrumentation

- Pretest shaping runs

- Test tolerance bands

- Accelerometer locations

- Data quality checks

- Overtest protection

- Fail Criteria

- Structural cracks and yielding

- Power interrupts

- Fail post test functional or deployment

- Parts fall off 


\section{Shock Test (cont)}

- Shock Test Criteria

- Peak g response from 100 to 10000 Hertz

- Or actual firing of explosively actuated devices or clamp band hardware (staging, separation, deployment releases)

- Impulse time history must be $<20 \mathrm{msec}$

- Damage potential

- Velocity $>50 \mathrm{in} / \mathrm{sec}=0.8 \times$ frequency

- Helpful Hints

- Unit test should precede vibration test

- System test should precede acoustic test

- Typically identifies design deficiencies

- Components too close to shock source

- Inadequate deployment clearances

- Breaks in wires, seals or crystals

- Relay chatter

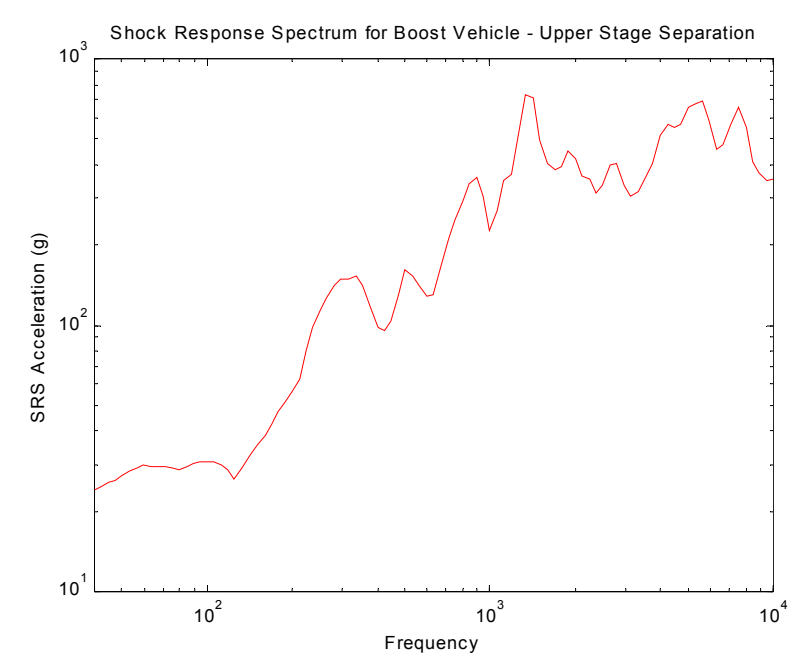




\section{Thermal Tests}

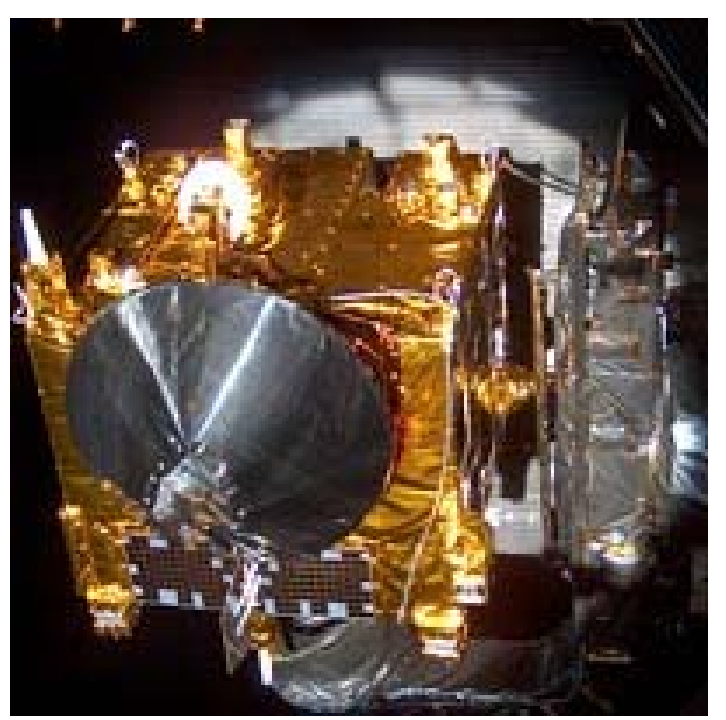

Photo courtesy of ESA

- A significant percentage of unit thermal cycling failures are parts related

- Verification of flight thermal control subsystem 


\section{Source of Thermal Environments}

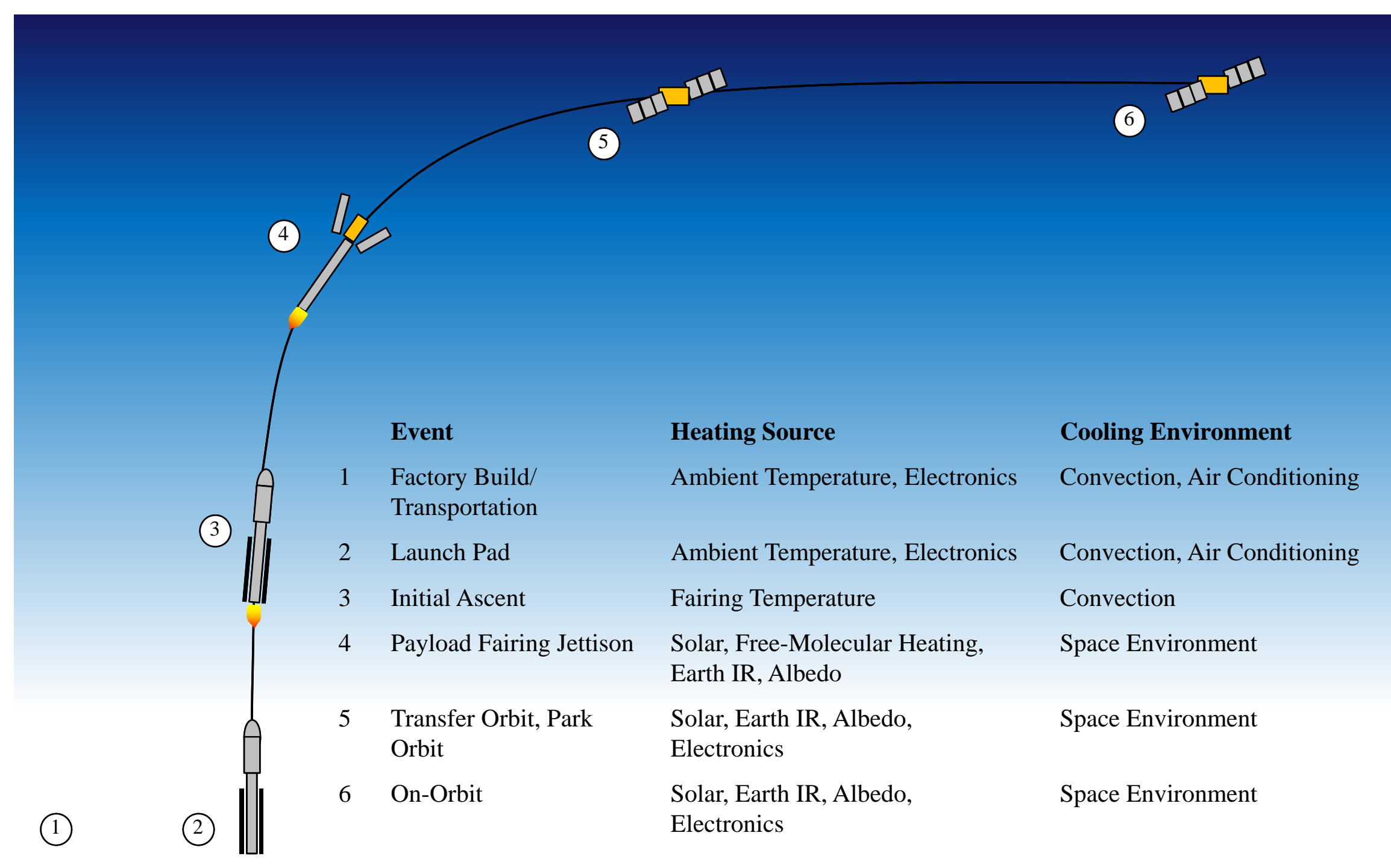




\section{Thermal Tests (cont)}

- Test Requirements

- Ambient pressure or vacuum

- Temperature extremes and range

- Operation/non-operation profile

- Dwell at temperature extremes

- Number of cycles

- Temperature transition rate

- Powered on or off

- Functional tests hot and cold

- Cold and hot starts

- Survival heater check out

- Redundant instrumentation

- Configuration

- Flight software

- On-orbit configuration
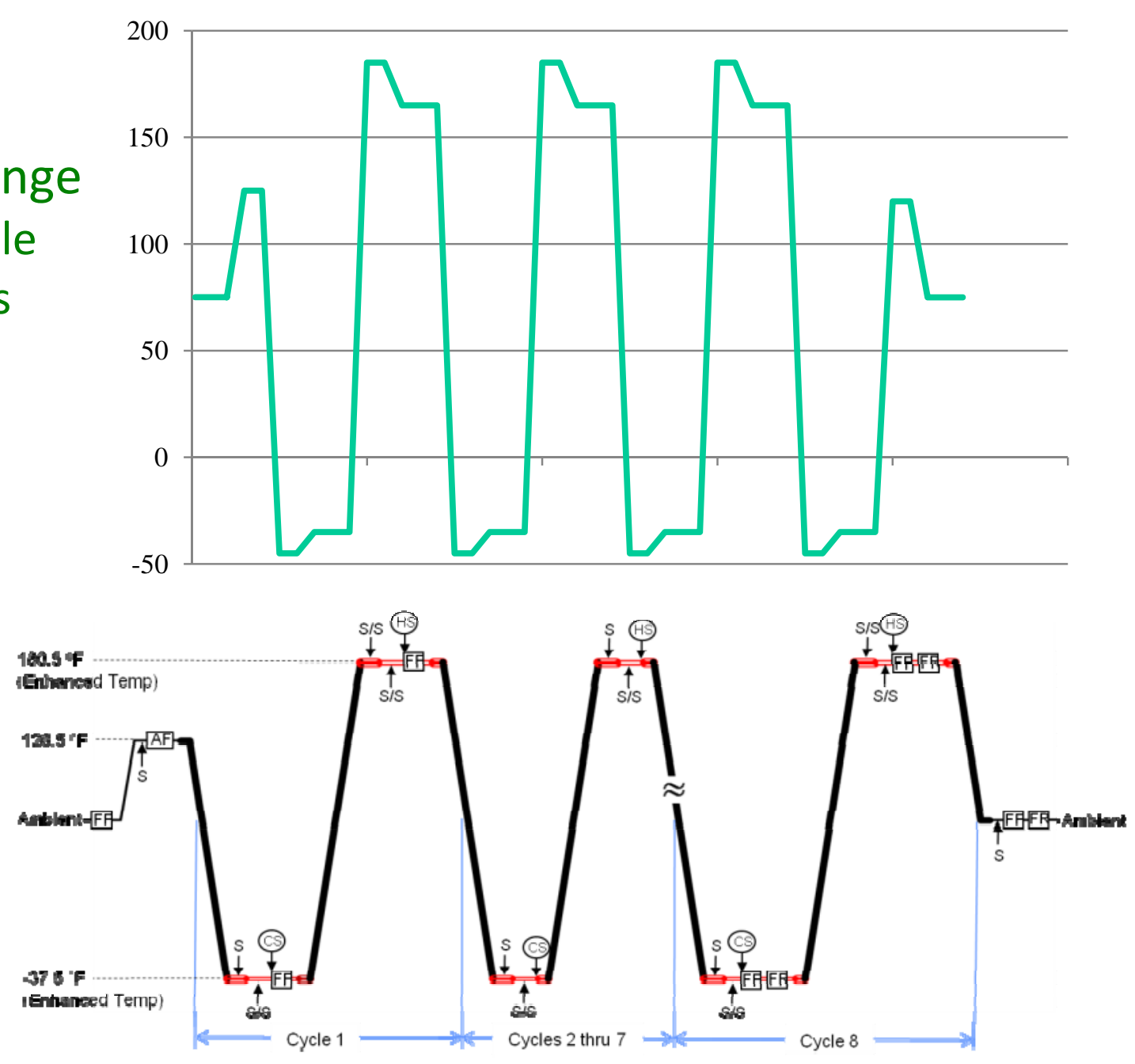


\section{Thermal Tests (cont)}

- Physics of failure

- Plastic strain/thermal expansion mismatch/ fatigue expressed by Coffin Manson/ Miners rule/ temperature - time related degradation

- Detection can be significant to 20 cycles thermal tests

- Helpful Hints

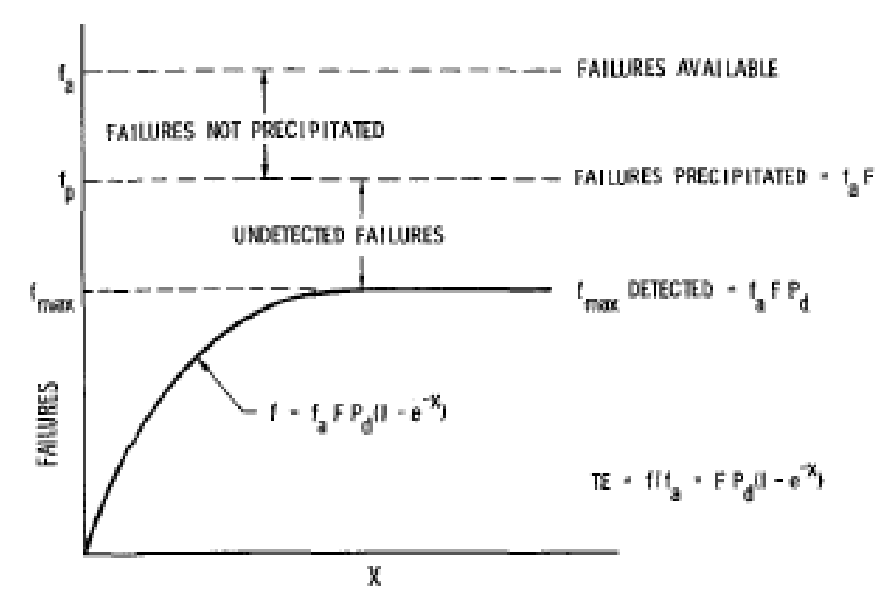

- Perform after vibration

- Vibration defects may precipitate in the thermal tests

- Start thermal testing at temperature above ambient (bake and dry out)

- End TVac at temperature higher than ambient

- A significant percentage of unit thermal failures are parts related 


\section{Survival and Turn-On}

- Verifies that the unit can survive extreme environments and operating conditions and subsequently perform within specification over a narrower environmental range

- Functions as part of the stress-screening process

- Demonstrates that the unit is well-designed, has good parts and materials, and is properly produced

- Demonstrates that the unit has survival and turn-on capability for anticipated and contingent environments

- Anticipated extreme environments

- Cold condition following transfer orbit

- Hot condition (possible operational)

- Contingent extreme environments

- Satellite damage or loss of attitude control or communication during ascent or transfer orbit

- Safemode conditions

- Failed heater or inadvertent operational mode 


\section{Powered On or Off During Transition?}

- MIL-STD-1540E requirements

- Unit on during transitions

- Cycle through operational modes

- Monitor perceptive parameters for failures and intermittents

- Cold start/Hot start

- Failure rates during screening doubled with the units powered on*

- IES guidelines recommends conducting thermal cycling tests with the power on

- Conclusion

- Screen is more effective with the UUT powered on

- More flaws are precipitated into failures

- Failures are easier to detect

\section{Significant Risk Trade to Test Without Power}

*Ref: K. L. Quart and I. Wong. Proceedings IEEE Reliability and Maintainability Symposium, IEEE, New York, 


\section{Vacuum or Ambient Pressure?}

\begin{tabular}{|l|c|c|}
\hline \multicolumn{1}{|c|}{ Test Objectives } & TVac & $\begin{array}{c}\text { Thermal } \\
\text { Cycle }\end{array}$ \\
\hline Flight-like Environment & $\mathbf{X}$ & \\
\hline Orbital Performance & $\mathbf{X}$ & \\
\hline Thermal Control & $\mathbf{X}$ & \\
\hline Arcing & $\mathbf{X}$ & \\
\hline Multipacting/Corona & $\mathbf{X}$ & \\
\hline Material Outgassing & $\mathbf{X}$ & \\
\hline Sensitivity to conduction & $\mathbf{x}$ & \\
\hline Electrical Intermittence & $\mathbf{X}$ & $\mathbf{X}$ \\
\hline Latent Defects/Failure Propagation & $\mathbf{X}$ & $\mathbf{X}$ \\
\hline Thermal Stress Effects & $\mathbf{X}$ & $\mathbf{X}$ \\
\hline Hardware Integration Verification & $\mathbf{X}$ & $\mathbf{X}$ \\
\hline Faster thermal transition rates & & $\mathbf{x}$ \\
\hline
\end{tabular}

\section{Thermal Vacuum is Not the Same as Thermal Cycle}




\section{Thermal Vacuum}

- Vacuum environment

- Qualification

- More cycles and larger temperature extremes

- Additional instrumentation

- Design objective and thermal balance emphasis

- Thermal control subsystem verification

- Achieve thermal equilibrium at both hot and cold temperatures

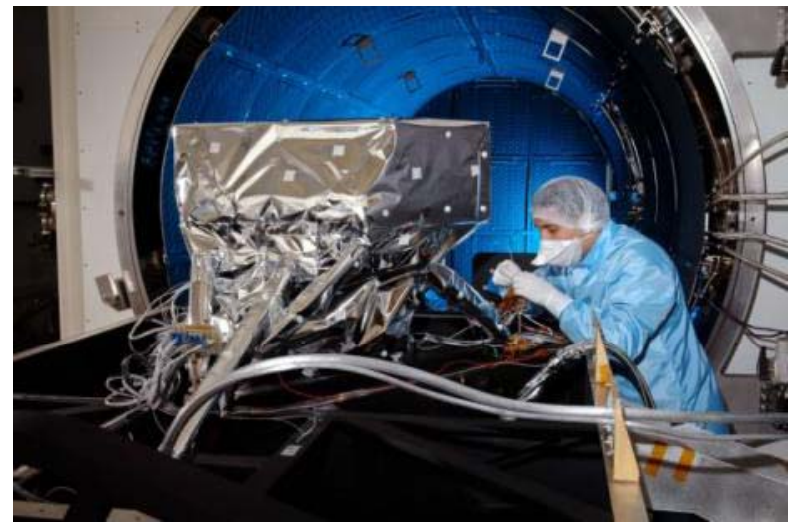

Photo courtesy of STFC

- Check emissivities of key surfaces and insulation blankets

- Check thermal conductance values (internal gradients)

- Check heat pipe and louver performance

- Primary and redundant thermostat/heater circuits:

- Activation commands and control authority verified

- Temperature control established, hot spots, cold spots, gradients determined

- Verify propulsion subsystem thermal performance 


\section{Thermal Balance Test}

- Qualification test to validate analytic thermal model

- Demonstrates and verifies the thermal control hardware and design in vacuum environment

- Typically three to eight mission phases simulated

- Cold phase, hot phase, heater verification, confirmation phase, transient phases

- Worst case combinations of seasons, equipment duty cycles, solar angles and eclipse conditions

- Exercise all important heat flow paths and response of temperature-sensitive and mission critical equipment

- Thermal vacuum environment simulated with heat load techniques and chamber cold wall

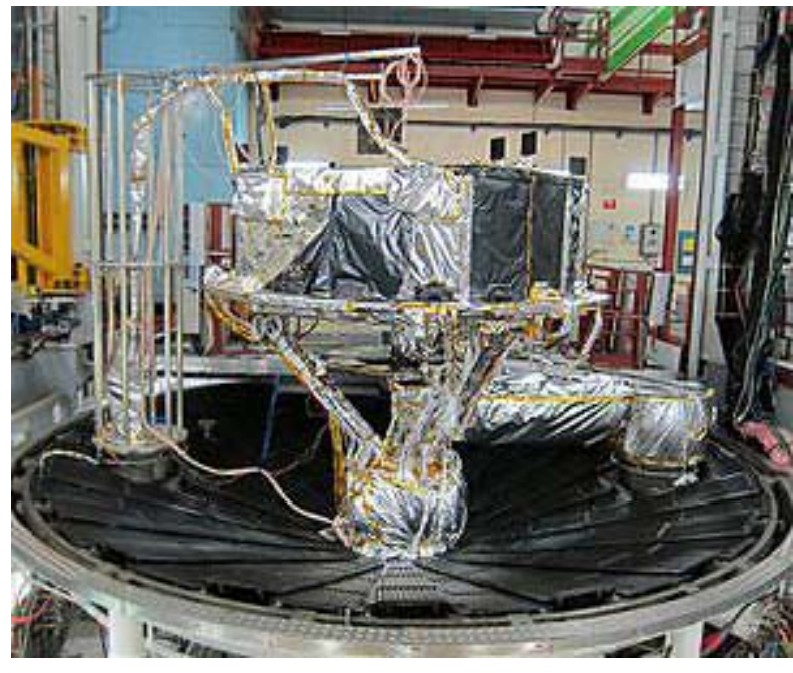

Photo courtesy of Astrium 


\section{Leak Test}

- Purpose

- Demonstrates the capability of pressurized components and hermetically sealed units to meet the specified design leakage rate requirements

- Hardware types

- Sealed electronic boxes

- Propulsion systems

- Pressure vessels

- Test Challenges

- Combined environments (temperature+vibration+pressure)

- Referee fluid/helium

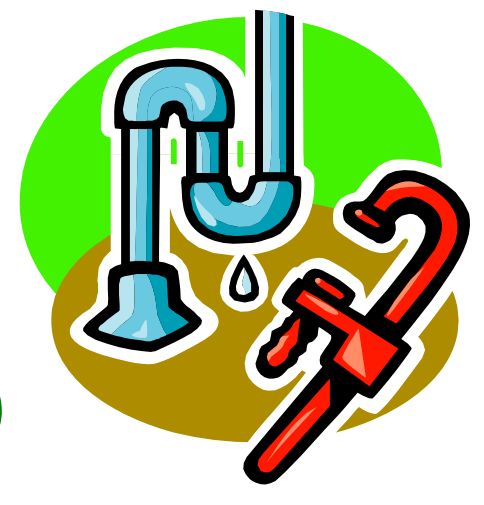

- Contamination

- Validation method

- Helpful Hints

- Differential pressure beyond MEOP

- Perform between environments

\section{Test for Leaks Early and Often}




\section{Pressure Test}

- Purpose

- Unit: demonstrate structural margin

- Subsystem/Vehicle: demonstrate flow, pressure and leakage rate within specification

- Environment

- Design burst pressure: Product of the maximum expected operating pressure (MEOP) and a burst factor

- MEOP: highest gage pressure that an item in a pressurized subsystem is required to experience during its service life

- Configuration

- Proof pressure: 1.1-1.5 x MEOP for 5 minutes

- Pressure cycle: 4 times expected cycles or 50 cycles

- Burst pressure: 1.25-5.0 x MEOP (non-flight UUT)

- Fail Criteria

- Bursts, permanent deformation, proper torquing, adequate regulation, etc

- Leakage at MEOP

Photo courtesy of The Aerospace Corporation

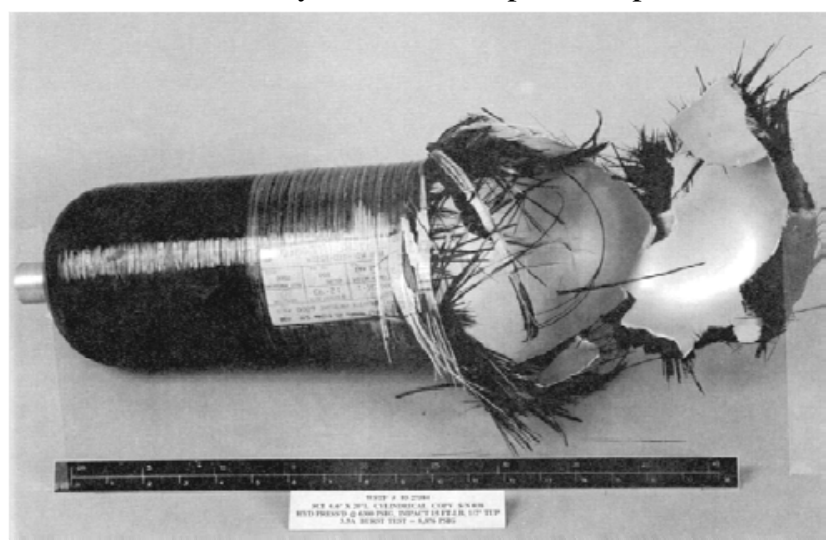




\section{Propulsion Pressure and Leak}

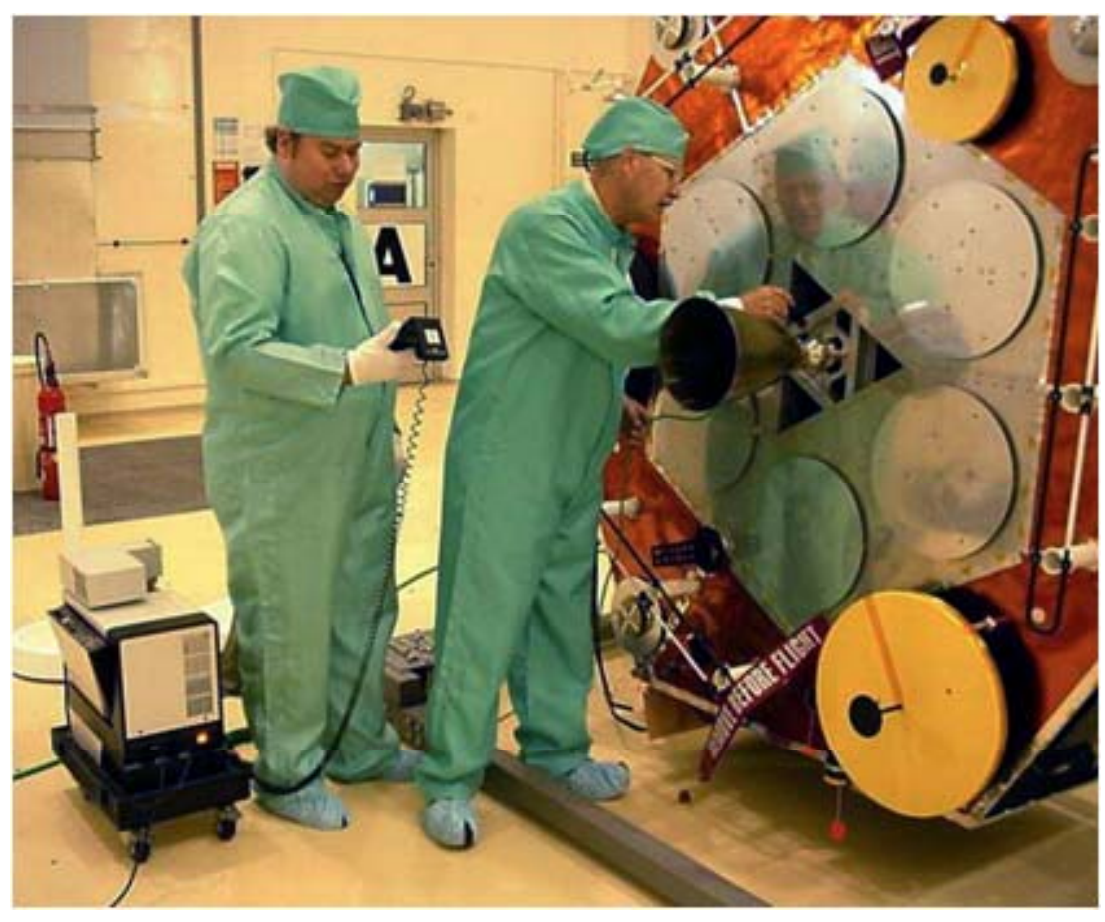

Photo courtesy of AMSAT-DL, Wilfried Gladisch 


\section{EME Tests}

- Purpose

- Hardware functions together in intended electromagnetic environment and is not source of interference

- Demonstrates satisfactory electrical and electronic equipment operation in conjunction with the expected electromagnetic radiation from other subsystems or equipment

- Configuration

- Flight configuration

- Uses all possible operational modes

- First system test is to verify requirement, 2nd test is to verify workmanship through environments leading to final flight configuration
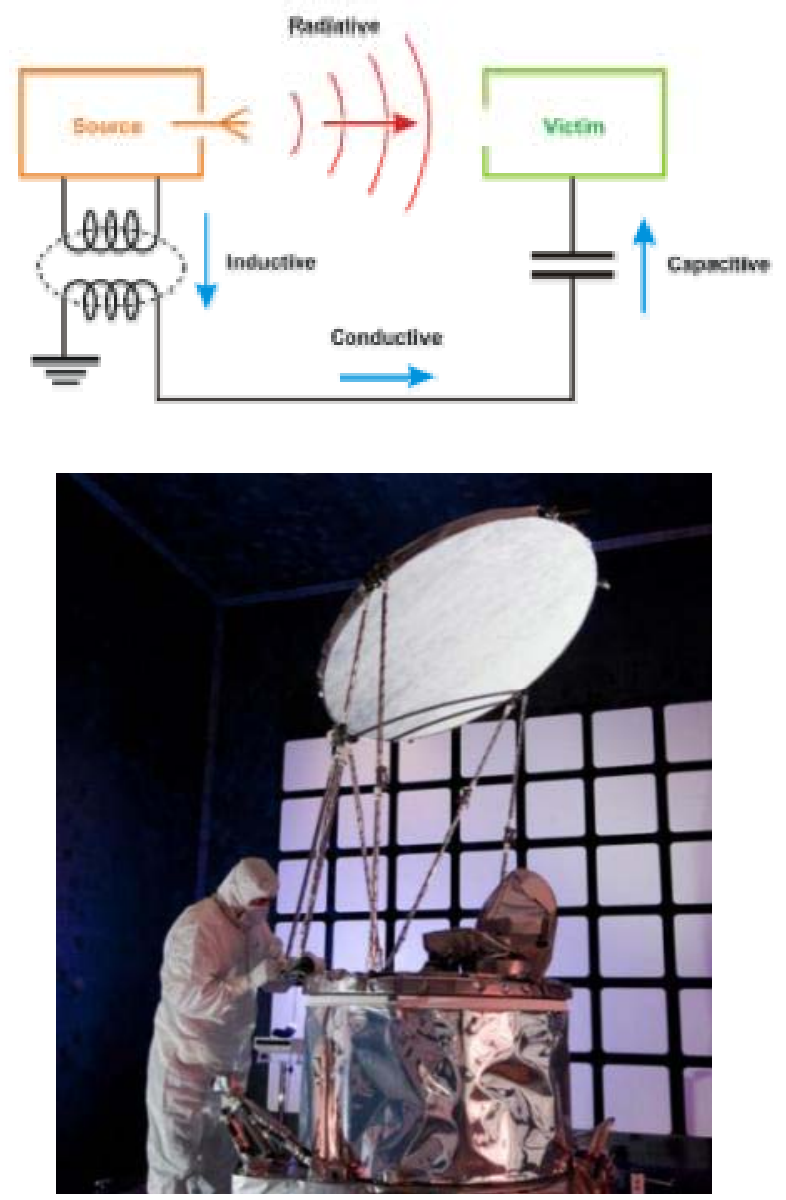

Photo courtesy of NASA 


\section{EME Tests (cont)}

- Test Types

- Electromagnetic Interference (EMI)

- Electromagnetic Compatability (EMC)

- Radio Frequency Interference (RFI)

- Radiated energy: through the air

- Conducted energy: along wires

- EME test families

- Radiated emissions and susceptibility (RE, RS)

- Conducted emissions and susceptibility

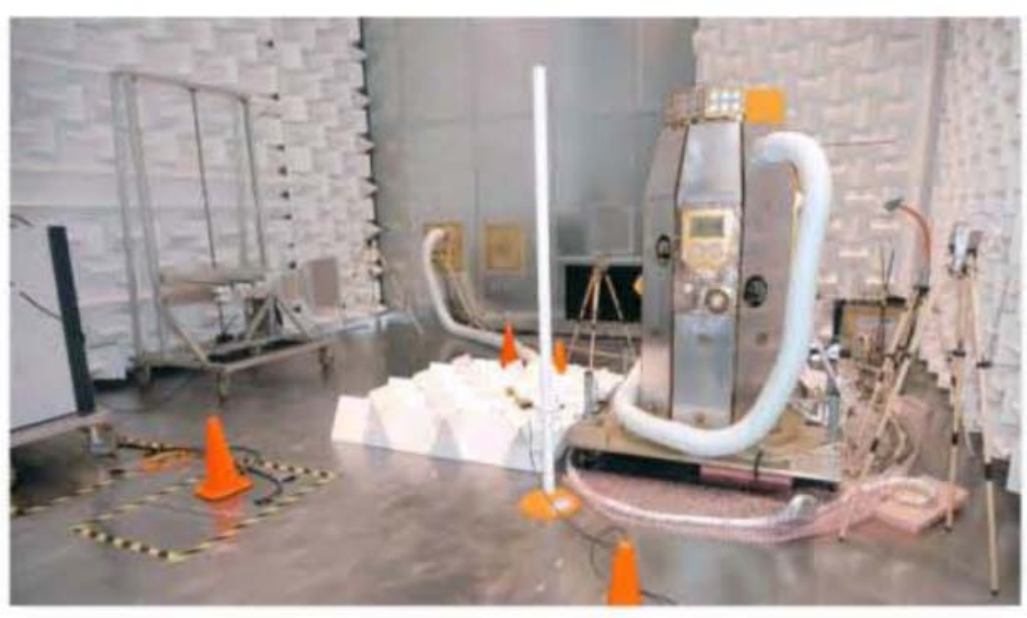

Photo courtesy of General Dynamics (CE, CS)

- Power quality

- Electrostatic discharge (ESD) 


\section{EME Tests (cont)}

- Fail Criteria

- Inability to command

- Loss of data

- Uncommanded response

- Bad housekeeping data

- Loss in signal:noise margin

- Helpful Hints

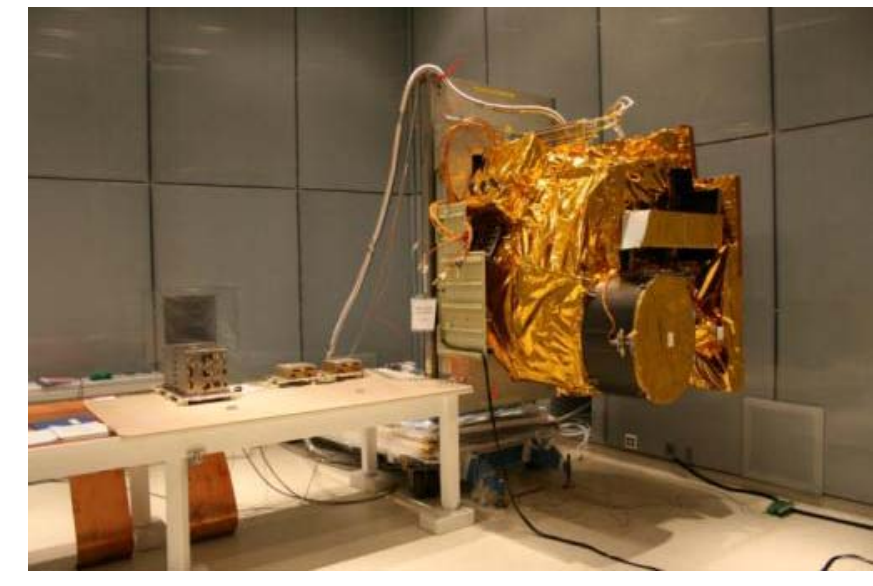

Photo courtesy of NOAA and NASA

- Use development tests to identify issues and inject into the design $=>$ don't patch

- 7 of 10 units don't meet original requirements

- Cost for discovery at next level factor of 8

- 1 in 10 spacecraft fail EMC at system level

- Pay particular attention to weaker units

- Best solution is a good design

\section{Follow Design Guidelines - Check Early if Critical}




\section{Life Test}

- Purpose

- Detect wearout, drift, or fatigue-type failure modes or performance degradation

- Exercise a unit through its cycle, stroke, or rotation during appropriate environment

- Test Requirements

- Pressurized structure/vessels duration: 50 cycles or 4 times predicted operating life or service cycles

- For structure not vibration qualified: 4 times life

- Other UUT: 2 times predicted operating life or service cycles

- Environment

- Ambient, thermal, and vacuum to evaluate wearout and drift failure modes

- Pressure, thermal, and vibration to evaluate fatigue-type failure modes 


\section{Life Testing}

- Configuration

- Flight quality

- Coupons made with the same process

- Helpful Hints

- Qualification test

- Special tests

- Battery charge/discharge

- Solar cell performance

- Bearings

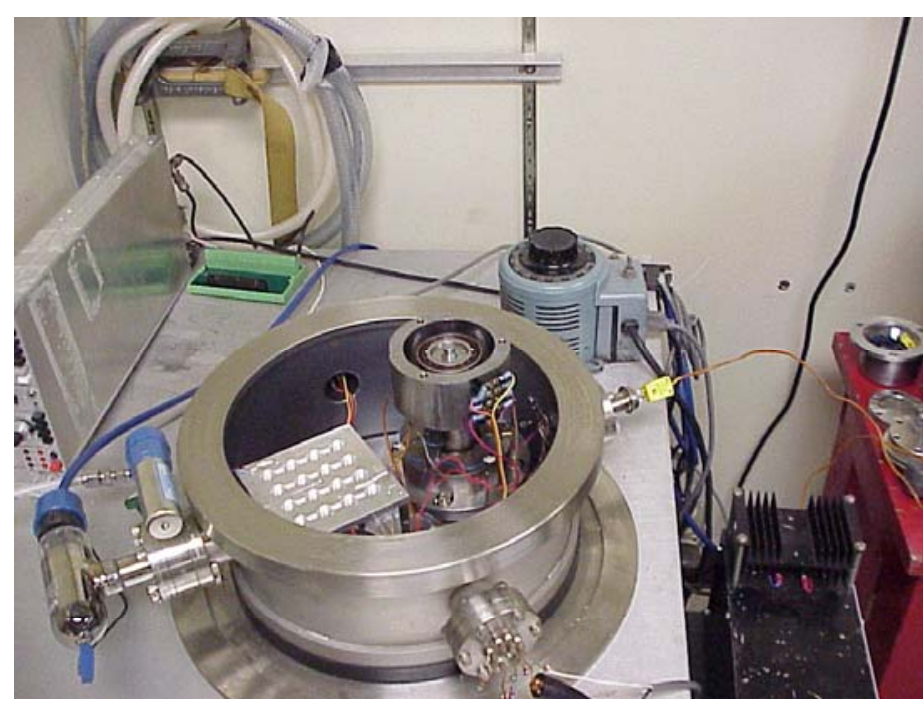

Bearing Under Test Aerospace Lab Ops Photo courtesy of Aerospace Corporation 


\section{A Real World Example of Problems from Unit Environmental Tests}

- Taken from a non-developmental (i.e., later in the block build) vehicle

- Shows that even late vehicles have unit problems

- Shows the kinds of events that correspond to the failures and delays

- Good visual for various readiness reviews 


\section{Power Supply \\ PN 35069-7 SN 2 (1 of 6)}

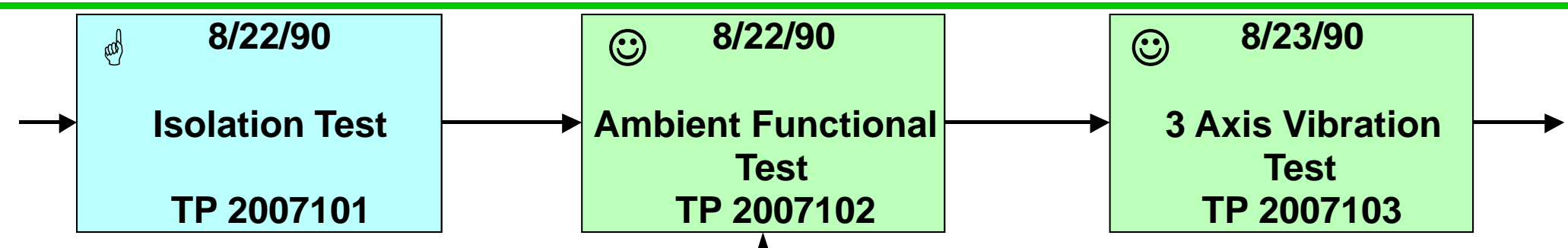

Alanned Events

Unplanned Events

Test Anomalies:

$\mathrm{D}$

TAR 0502 - Unit Failed

Standby Power Test.

Error in slice A.

Tester Wiring. No

Overstress to the UUT.

Repaired the Tester.

Repeated Test.

\begin{tabular}{|c|c|c|c|}
\hline \multirow{3}{*}{ (2) } & \multicolumn{2}{|c|}{ LEGEND } & \\
\hline & First Acceptance Test & Other Work In Progress & \\
\hline & Good Acceptance Test & Last Unit Invasion & \\
\hline 通 & Good Acceptance Retest & Last Accept Test/Completed Unit & Pr \\
\hline (1) & Failed Acceptance Test/Rework & Insignificant Unplanned Events & $\mathrm{D}$ \\
\hline
\end{tabular}




\section{Power Supply \\ PN 35069-7 SN 2 (2 of 6)}

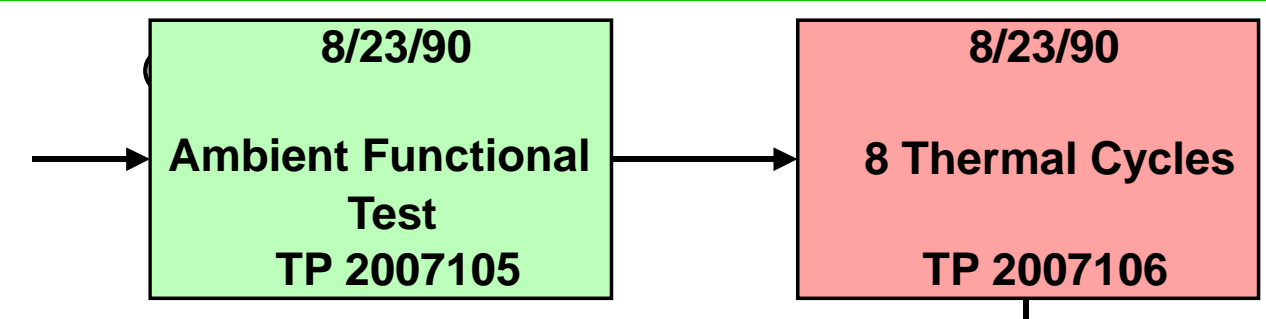

APlanned Events

Unplanned Events

Notes :

1. See FRB 21045 for Detailed Analysis and Corrective Action. 


\section{Power Supply \\ PN 35069-7 SN 2 (3 of 6)}

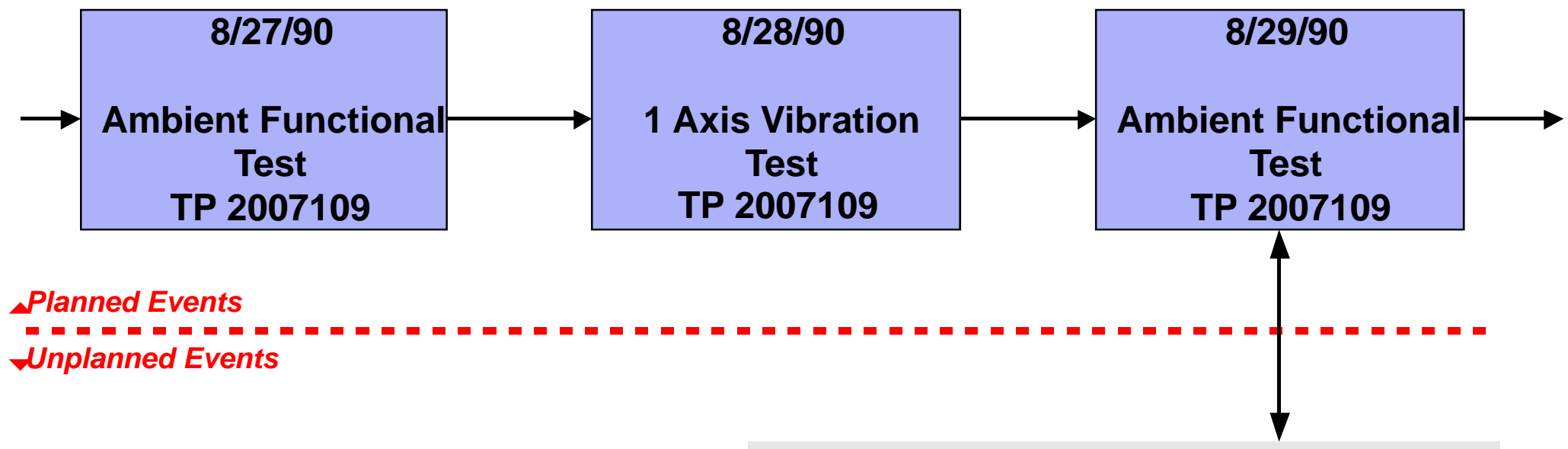

Test Anomalies:

囚TAR 0525 - Unit Wouldn't Power on. Test Cable Not Seated. No Overstress to the UUT. Cable Reseated. Test Repeated.

XITAR 0526 - Erroneous Current Reading. Tech Misinterpreted the Procedure. No Overstress to the UUT. Test Continued.

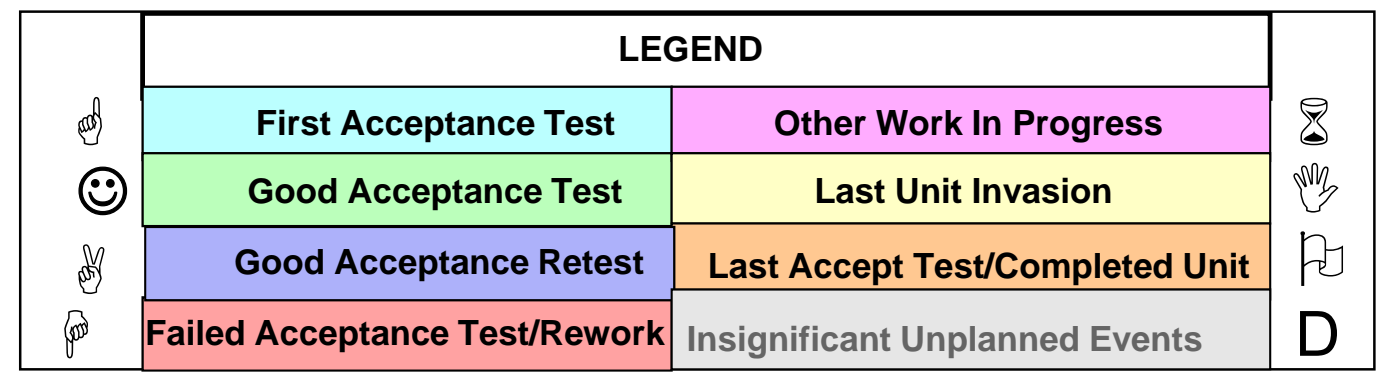




\section{Power Supply \\ PN 35069-7 SN 2 (4 of 6)}
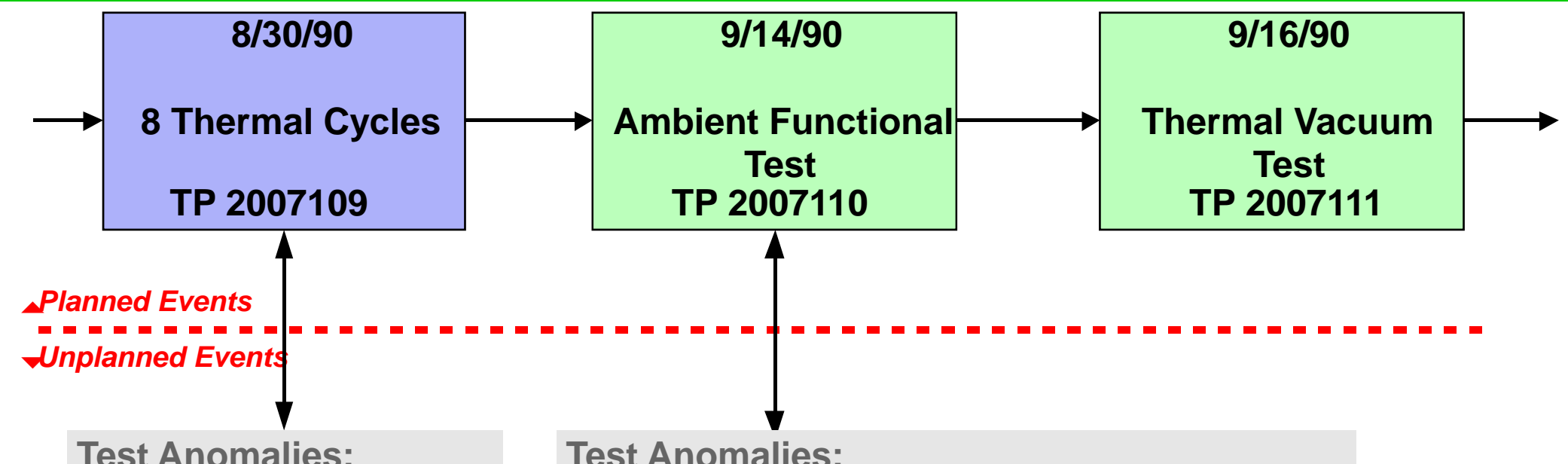

凶 TAR 0533 - Distorted

Wave Forms. Tester

Problem. No

Overstress to the

UUT. Repaired Tester.

Continued Test.

区 TAR 0537 - During

TC\#3 the Wrong

Redundancy was on.

Tech Error. No

Overstress to the

UUT. Repeated TC\#3.

XTAR 0553 - Side "A" Section Failed to

Command on. Test Equipment Anomaly. No

Overstress to the UUT. Test Repeated.

XITAR 0586 - Unit Failed Temp Output Test.

Two Test Cables Miswired. Reworked

Cables. No Overstress to the UUT. Test

Repeated.

\begin{tabular}{|c|c|c|c|}
\hline \multirow{3}{*}{ () } & \multicolumn{2}{|c|}{ LEGEND } & \multirow[b]{2}{*}{8} \\
\hline & First Acceptance Test & Other Work In Progress & \\
\hline & Good Acceptance Test & Last Unit Invasion & M \\
\hline an & Good Acceptance Retest & Last Accept Test/Completed Unit & De \\
\hline (300) & Failed Acceptance Test/Rework & Insignificant Unplanned Events & $\mathrm{D}$ \\
\hline
\end{tabular}

Note: Shadowed Boxes Represent Significant Unplanned Events 


\section{Power Supply \\ PN 35069-7 SN 2 (5 of 6)}

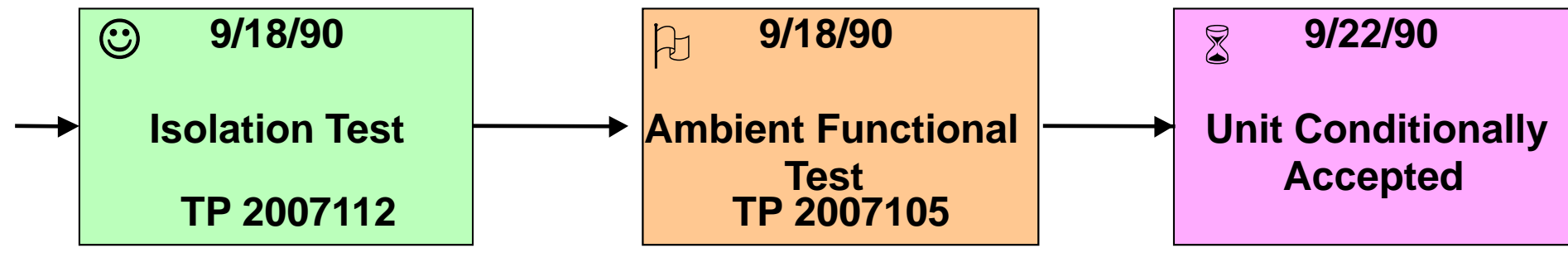

$\triangle$ Planned Events

Unplanned Events

\begin{tabular}{|c|c|c|c|}
\hline \multirow{3}{*}{$\begin{array}{l}\text { (i) } \\
\text { (-) }\end{array}$} & \multicolumn{2}{|c|}{ LEGEND } & \multirow[b]{2}{*}{8} \\
\hline & First Acceptance Test & Other Work In Progress & \\
\hline & Good Acceptance Test & Last Unit Invasion & \\
\hline ast & Good Acceptance Retest & Last Accept Test/Completed Unit & \\
\hline (1000) & Failed Acceptance Test/Rework & Insignificant Unplanned Events & $\mathrm{D}$ \\
\hline
\end{tabular}

Note: Shadowed Boxes Represent Significant Unplanned Events 


\section{Power Supply \\ PN 35069-7 SN 2 (6 of 6)}

\begin{tabular}{|c|c|c|}
\hline $9 / 26 / 90$ & $10 / 9 / 90$ & 10/9/90 \\
\hline $\begin{array}{l}\text { Unit Installed on } \\
\text { Sensor } 019 \\
\text { TP } 2007113\end{array}$ & $\begin{array}{c}\text { Redlines } \\
\text { Incorporated }\end{array}$ & Unit Accepted \\
\hline
\end{tabular}

Planned Events

Unplanned Events

\section{$\underline{\text { Recap }}$}

Functional, Vibration, Thermal Cycling, Thermal Vacuum

- Total test time: $8 / 22-10 / 9=7$ weeks

- \# Vibration test/retests $=2$

- \# Thermal cycle test/retest=2

- \# partial retests = 5

\begin{tabular}{|c|c|c|c|}
\hline \multirow{3}{*}{$\begin{array}{l}\text { (i) } \\
\text { (-) }\end{array}$} & \multicolumn{2}{|c|}{ LEGEND } & \multirow[b]{2}{*}{8} \\
\hline & First Acceptance Test & Other Work In Progress & \\
\hline & Good Acceptance Test & Last Unit Invasion & \\
\hline ast & Good Acceptance Retest & Last Accept Test/Completed Unit & \\
\hline (1000) & Failed Acceptance Test/Rework & Insignificant Unplanned Events & $\mathrm{D}$ \\
\hline
\end{tabular}




\section{SOFTWARE TESTING}




\section{Software is an Integrated Subsystem}

- Flight software should be treated as an integrated subsystem

- Often thought of as independent subsystem - it isn't!

- Resides on hardware in different subsystems

- ex., GN\&C, comm, TCS, payload, EPS

- Avionics is condensing boxes into cards and cards are condensing into chips

- Lots of software and hardware interfaces

- Need (new) agile and thorough systems engineering and verification techniques and tools

- Incremental builds

- Build and test is not complete until the final build

- Identify subset of requirements for each build

- Need to test software developed in current build with other software developed in previous builds

"Software has become the last refuge for fixing problems"

- Steve Jolly, MRO Program Manager 


\section{All Software has Bugs}

- It costs more to fix bugs the later they are discovered

- ex., 10x more to fix a bug in the coding phase than the requirements phase

- Requirements - not coding - is a major source of software errors

- Poorly stated, ambiguous

- Changing/creeping

- Nonfunctional

- Omitted

- Peer reviews and product evaluation required prior to testing

- Formal process

- Come prepared

- Document results 


\section{Flight Software Failures}

- Satellite loses ground contact after several hours

- An ACS software sign error kept the spacecraft from pointing at the Sun

- Cause: lack of end-to-end testing

- Launch vehicle delivers satellite to wrong orbit

- Entered flight control parameter order of magnitude smaller than test parameter

- Cause: testing was not performed with the flight software

"Reducing the frequency of testing to cut costs should be avoided. Many recent launch vehicle failures and mission mishaps could have been prevented had testing not been shortchanged." "...inadequate reviews, poor risk management and insufficient testing/verification were each found in six of eight separate mission failure investigations."

Report on Project Management in NASA. March 13, 2000 


\section{Simulators and Test Beds}

- Flight-like hardware

- Simulate hardware interfaces

- Simulate signal interfaces

- Simulate orbital condition

- Flight software and databases

- Test software and databases

- Used to verify potential degrading requirements

- Scenarios

- Negative testing

- Stress testing

- Off nominal testing

- Contingencies
- Understand

- Differences

- Limitations

- Test specific responses

- Validate simulator/test bed

- Control the configuration - Treat like it is flight

- Document anomalies

\section{Validated and Configuration Controlled Simulators and Test Beds are Critical}




\section{Software Verification - NOT Just Test}

- All verification methods need to be utilized

- Do not rely on (and wait for) testing to identify the bugs

- Cannot perform 100\% testing

- Space software is complex and run in interdependent environments

- Time and money run out before all testing can be performed

- Prioritize test objectives and optimize test procedures for important and critical tests

- Good risk analysis skills essential

- Critical requirements/tests

- Safety - failure leads to hazard of system or personnel

- Security - failure leads to breach of system security

- "ility" - failure adversely affects dependability, reliability, maintainability, availability

- Other mission critical requirement - failure adversely affects Key Performance Parameter (KPP) 


\section{Software Test Preparation}

- Preparation for test

- Multi-discipline review of test cases and test procedures

- Recommend engineering tests on functionally equivalent units and/or dry run (informal test) prior to performing run for record

"Although programmers, testers, and programming managers know that code must be designed and tested, many appear to be unaware that tests themselves must be designed and tested - designed by a process no less rigorus and no less controlled that that used for code."*

- Test case

- Inputs

- Environments

- Traceabilty to requirements and design information

- Execution details (ex., procedure)

- Expected results and success criteria

- Potential hardware risks/issues and appropriate actions to take

- Configuration controlled 


\section{Tests Shall Be}

- Repeateable

- Identical hardware and software should generate identical results

- Complete

- Objective evidence of compliance to allocated requirements, design elements, and coverage criteria

- Configuration controlled

- Software input and output products (ex. databases), environments (ex., test equipment), procedures, scripts, and test cases

- Independent

- After unit level, test must be independently conducted (someone other than the developer of the code)

\section{Test Thoroughly at the Correct Levels}




\section{Definitions for Software Testing}

- Blackbox testing - requirements based testing

- Tester focuses on requirements and has no knowledge of internal structure

- aka functional testing

- Branch testing - every instruction in each conditional statement

- Graybox testing - mixture of whitebox and blackbox testing

- Functional testing where tester has sufficient knowledge of software's structure to drive testing to focus on critical and "hard to reach" paths

- aka mixed testing

- Negative testing - show something is not met or doesn't work

- Outside of normal or "illegal" input

- Nominal testing - uses normal parameters

- aka positive testing 


\section{Definitions for Software Testing (cont)}

- Performance testing - focused on requirements related to system execution and speed

- Path testing - every set of conditional statements of branches

- Regression testing - retest of previously tested software after a change to ensure (still) performs as it should

- Scenario based testing - demonstrate operational capabilities and functions based on the concept of operations for nominal and offnominal conditions

- Clear what stimulus and response of interest are

- Stress testing - uses simulated levels at and beyond limits

- ex., ranges, rates, workload

- Whitebox testing - code based testing

- Tester has knowledge of software's structure

- NOT debugging 


\section{Software Testing}
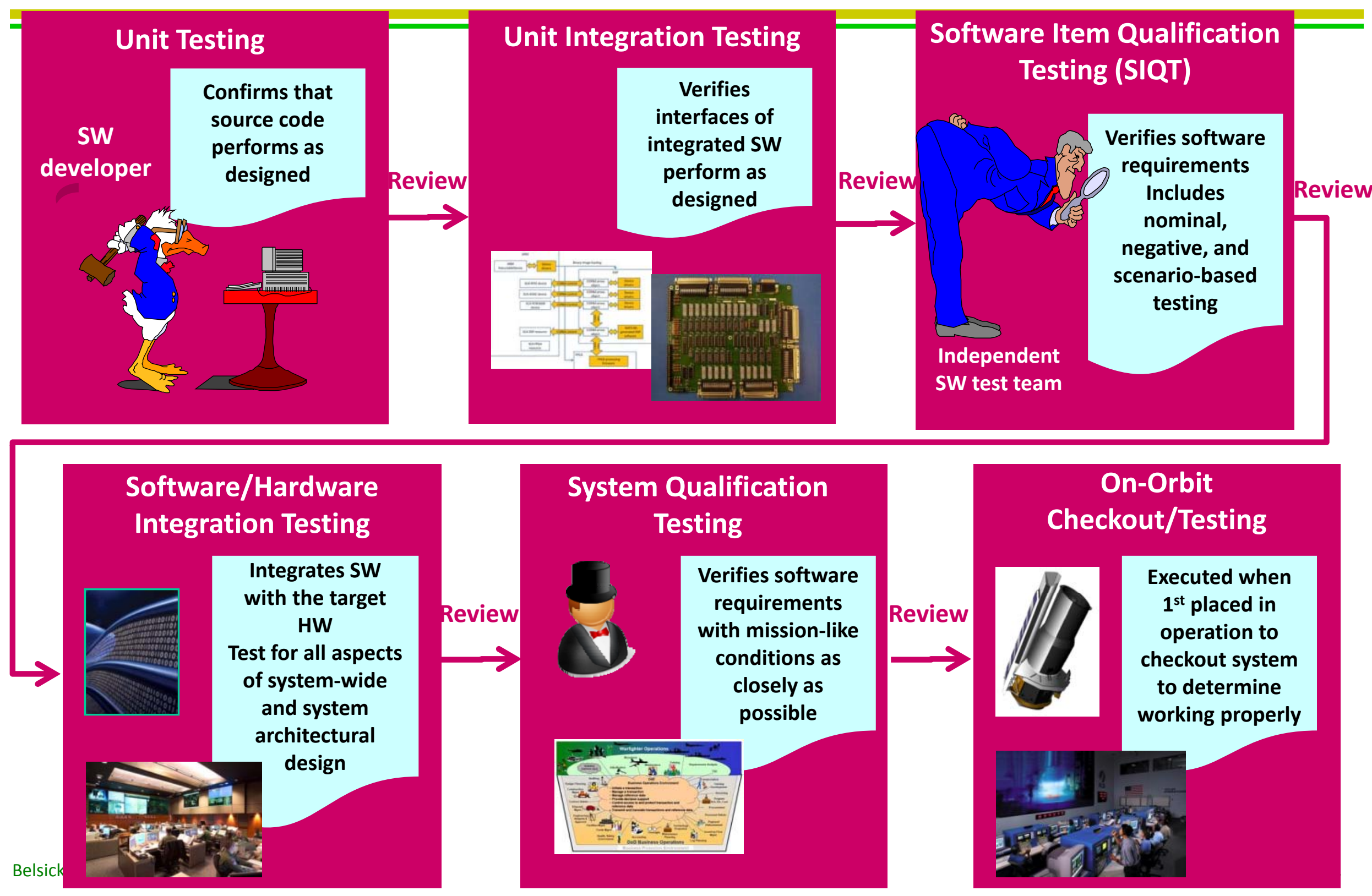


\section{Software Unit Testing}

- Purpose

- Single compilation element which performs low level function, subfunction, or task

- Verifies source code modules perform as designed

- Test cases shall cover (at a minimum)

- Unit design including correct execution of all statements and branches

- All error and exception handling

- All software unit interfaces including limits and boundary conditions

- Start-up, termination, and restart

- All algorithms

- Test legacy reuse software if

- Modified reuse software units

- Track record indicates potential problems

- Even if not modified

- Critical reuse software units

- Even if not modified

- Perform on simulators and test hardware by software developer 


\section{Software Unit Integration and Testing}

- Purpose

- Integrate 2 or more software units and test to make sure it works as intended

- Testing against the software design

- Whitebox testing of logical paths through software, boundary testing, and error handling

- Test cases shall cover (at a minimum)

- Correct execution of all interfaces between software units including limit and boundary conditions

- Integrated error and exception handling across the software units

- All end-to-end functional capabilities

- All allocated software requirements

- Performance testing including operational input and output data rates and timing and accuracy requirements

- Stress testing including worst-case scenarios

- ex., fault tolerance, fail over, data capture and reporting

- Resource utilization measurement

- ex., CPU, memory, storage, bandwidth

- Perform on target hardware in configuration close to operational by software developer

- All reuse software including modified and unmodified legacy reuse and COTS shall undergo unit integration and testing 


\section{Software Item Qualification Testing (SIQT)}

- Purpose

- Demonstrate that software item requirements have been met

- Blackbox testing, demonstrating operational functionality, proper input acceptance and output generation, and data integrity

- Test cases shall cover (at a minimum)

- Verification of all software requirements under operation environment conditions

- ex., operational data constants, operational input and output data rates, operational scenarios, target hardware configurations

- Verification of all software interface requirements using actual interfaces or high-fidelity simulation

- Verification of all software specialty engineering requirements including software reliabilty requirements and fault detection, isolation, and recovery

- ex., supportability, testability, dependability/reliability/maintainability/availability, safety, security, human systems integration

- Stress testing including worst-case scenarios

- Resource utilization measurment

- Perform on target hardware as close as possible to the operational target hardware in operational configuration

- Independent verification and validation (IV\&V)

- Performed by a software test organization independent of the developers

- All software requirements shall be verified by SIQT regardless of new, reuse, or COTS 


\section{SW/HW Integration \& Testing}

- Purpose

- Integrate software items with interfacing (target) hardware and software items and test for all aspects of system-wide and system architectural design

- Includes non-developmental software (e.g., COTS, reuse) and developed software

- All reuse software including modified and unmodified legacy reuse and COTs shall undergo software/hardware integration and testing

- Whitebox testing of the software/hardware design

- Test cases shall cover (at a minimum)

- Correct execution of all SW-to-SW and SW-to-HW interfaces including limit and boundary conditions

- Integrated error and exception handling

- End-to-end funtional capabilities

- All allocated software requirements

- Performance testing including worst-case scenarios

- Start-up, termination, restart

- Fault detection, isolation, and recovery handling

- Resource utilization measurement

- Perform on target hardware in configuration close to operational

- Include actual ground equipment

- Performed by independent element/segment/system integration team(s) with support from software and hardware IPTs

- Hardware and software are pretested separately to find and eliminate errors 


\section{Software System Qualification Testing}

- Purpose

- Demonstrate system requirements have been met

- Uses already qualified software and hardware

- Testing represents mission-like conditions as closely as possible

- Blackbox testing against the prime/critical item, element, segment, and system requirements

- Includes software item and software interface requirements

- Test cases shall cover (at a minimum)

- Requirements in the system specification, segment specifications, and all other levels of requirements between the system and segment specifications and the software requirements in the specification tree including interface requirements at all levels

- Perform on operational target hardware in operational configuration

- Performed by independent element/segment/system integration team(s) with support from software and hardware IPTs

- Process

- Dry run system test cases and procedures to ensure they are complete and accurate

- Update system test cases and procedures

- Perform "run for record" test 


\section{Definitions for Software Anomalies}

- Defect - flaw in system or software product that is discovered through inspection process

- Note: residual defects not discovered can lead to faults, failures, and anomalies

- Fault - flaw in system or software product that is discovered through a test process

- Failure - inability of a system or component to perform its required functions within specified performance requirements

- Anomaly - any condition that deviates from expectations based on requirements specifications, design documents, user documents, standards, etc, or someone's perception or experience

- Anomalies may be found during, but not limited to, review, test, analysis, compilation, or use of software or applicable documentation 


\section{Software Anomaly Classification by Severity}

\section{Severity Applies if a problem could:}

1 a. Prevent the accomplishment of an operational or mission essential capability

b. Jeopardize safety, security, or other requirement designated "critical"

2 a. Adversely affect the accomplishment of an operational or mission essential capability and no work-around solution is known

b. Adversely affect technical, cost, or schedule risks to the project or to life cycle support of the system, and no work-around solution is known

3 a. Adversely affect the accomplishment of an operational or mission essential capability but a work-around solution is known

b. Adversely affect technical, cost, or schedule risks to the project or to life cycle support of the system, but a work-around solution is known

4 a. Result in user/operator inconvenience or annoyance but does not affect a required operational or mission essential capaility

b. Result in inconvenience or annoynace for development or maintenance personnel, but does not prevent the accomplishment of those responsibilities

5 Any other effect

Ref: SMC-S-012 Software Development for Space Systems - Appendix C 


\section{Common Mistakes}

- Inadequate software test planning

- Need to develop new agile and thorough methods

- Inadequate (or no) unit testing by the software developers

- Latent errors in code are sent to unit integration

- Insufficiently tested or untested modules are promoted

- Error isolation and correction is impossible

- Perceptiveness problem

- Integration tests fail to uncover errors

- Prove what's known, not test for what isn't

- Test cases are written to show that the software works, but do not provide sufficient error testing

- Need negative testing

- Software qualification not performed on target hardware configuration

- Requirement verification may be invalid, especially performance requirements

- Software qualification performed after delivery

- No regression testing of requirements verified in previous builds 


\section{Pieces of the Puzzle}

- Exponential Growth in Flight Software

- software development can exceed hardware development time

- Qualification takes even longer

\section{IExponential}

IGrowth in

IFlight Software

- How on Critical

Path

Flight and Payload Software Equivalent Lines of Code (KSLOC)

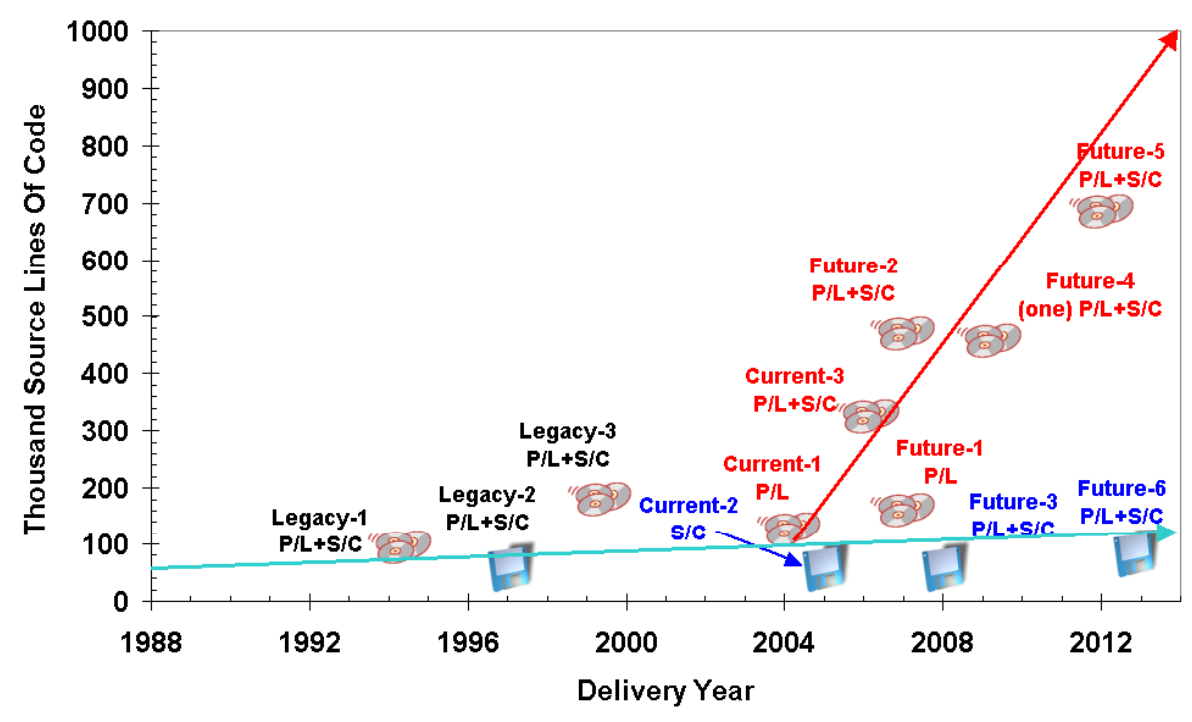

\section{Software Deliveries Now on Critical Path in Space Vehicle Development}




\section{Pieces of the Puzzle}

Exponential

Growth In

Flight Software

- How on Critical

Path flight software related anomalies

- In test

- In flight

\section{It's Better to Find Them in Test than in Flight!}

Need to Develop and Use Different Practices

\section{Which Hegns}

Exponential

Growth In

Flight Software

Anomalies

- In test

- In flifint
Survey of best military software contains 5 defects/KSLOC, suggests $5 \%$ manifest into anomalies

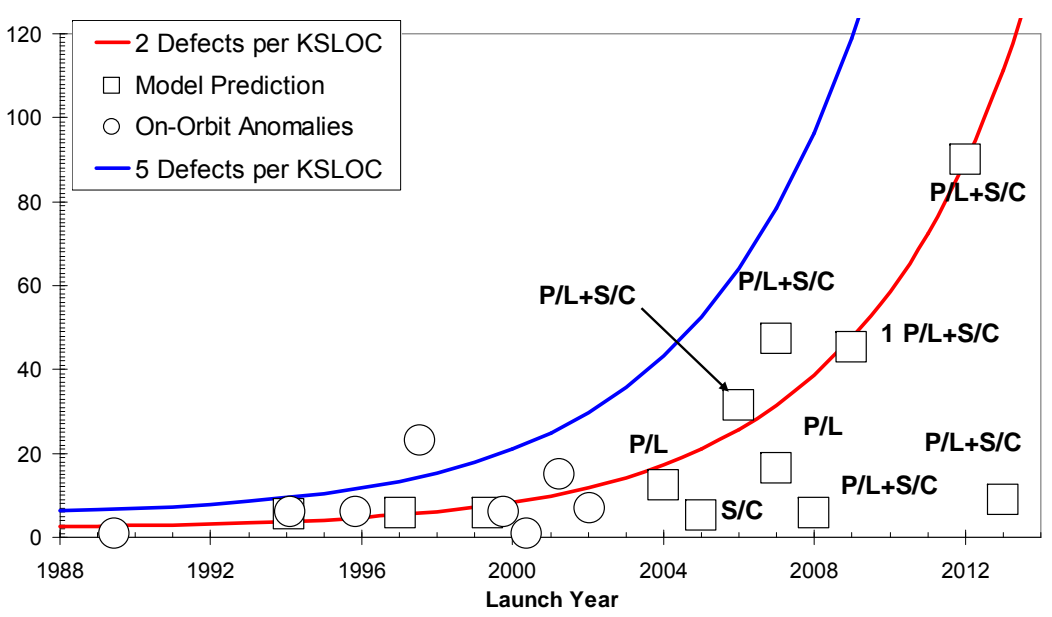




\section{Pieces of the Puzzle}

- On-orbit anomaly histories show software related anomalies can

\section{Exponential}

Growth In

Flight Software

- How on Critical

Path and do damage space systems

- Effective testing minimizes the anomalies encountered in flight

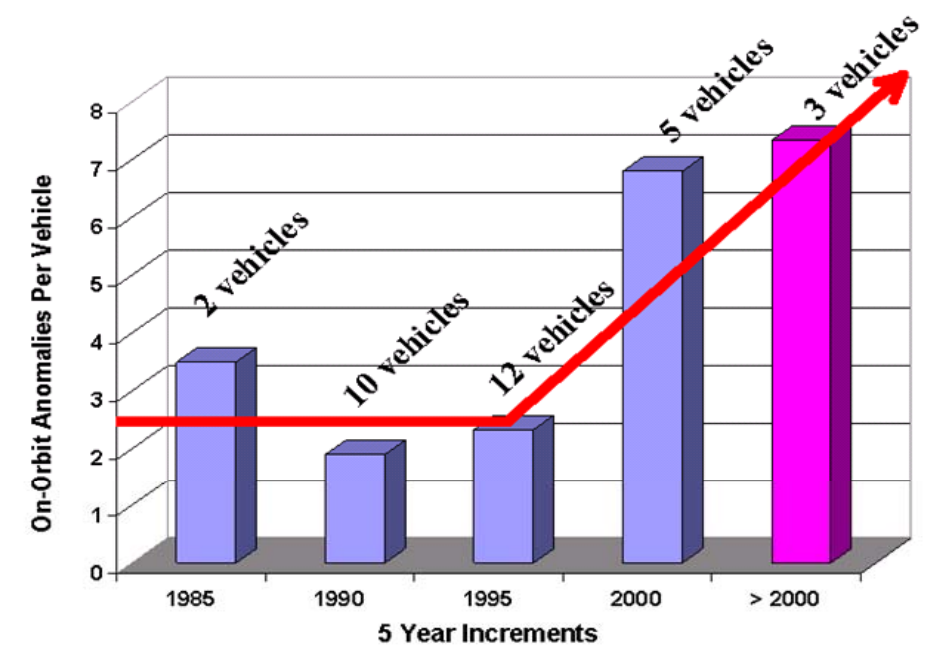




\section{Pieces of the Puzzle}

- Effective system level testing means using the fully integrated flight system including qualified flight software

- Would you test without the flight computer?

- Understand and validate the simulators and test beds

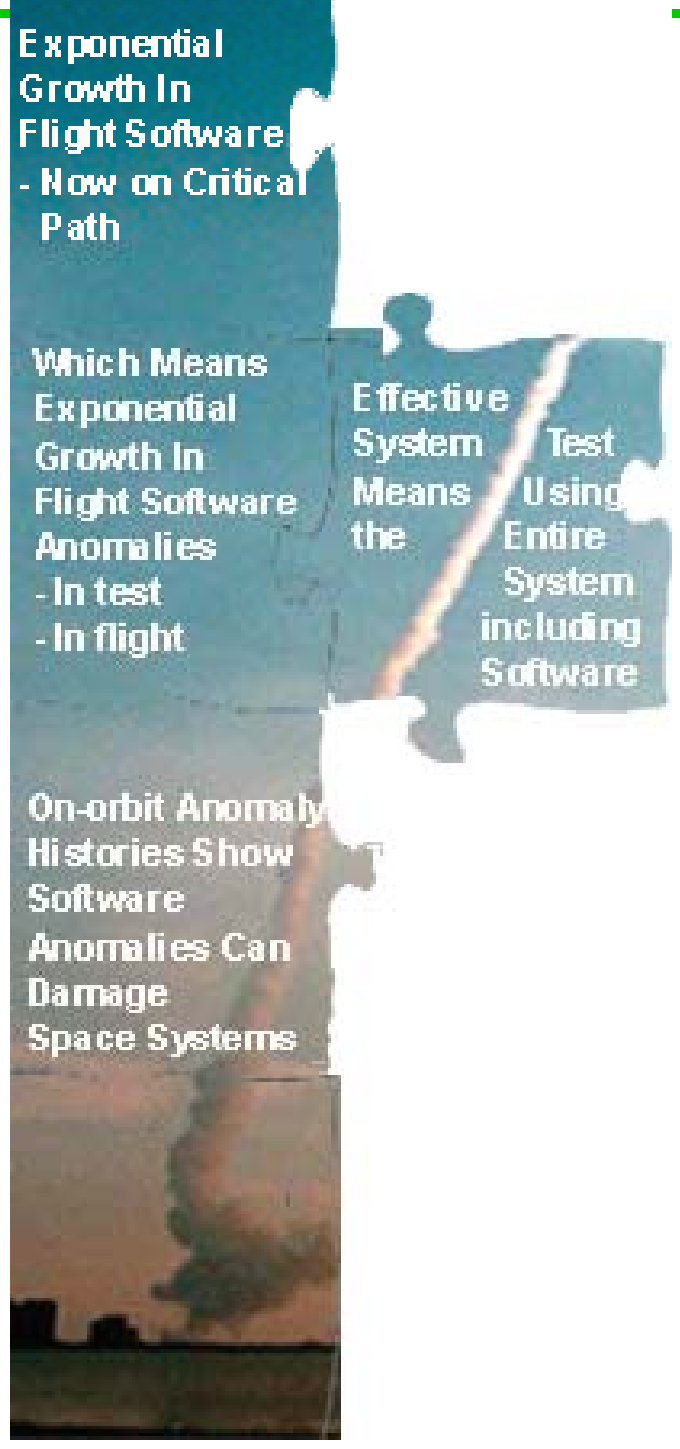




\section{Pieces of the Puzzle}

- Thermal vacuum is the closest environment test to flight-like

- Test Like You Fly

- Escapes

- $42 \%$ of the failures in TVac were escapes from previous tests*

- Thermal control subsystem

- Thermal control dependencies can only be checked during thermal testing

- Temperature dependent hardware

- ex., cryo payloads
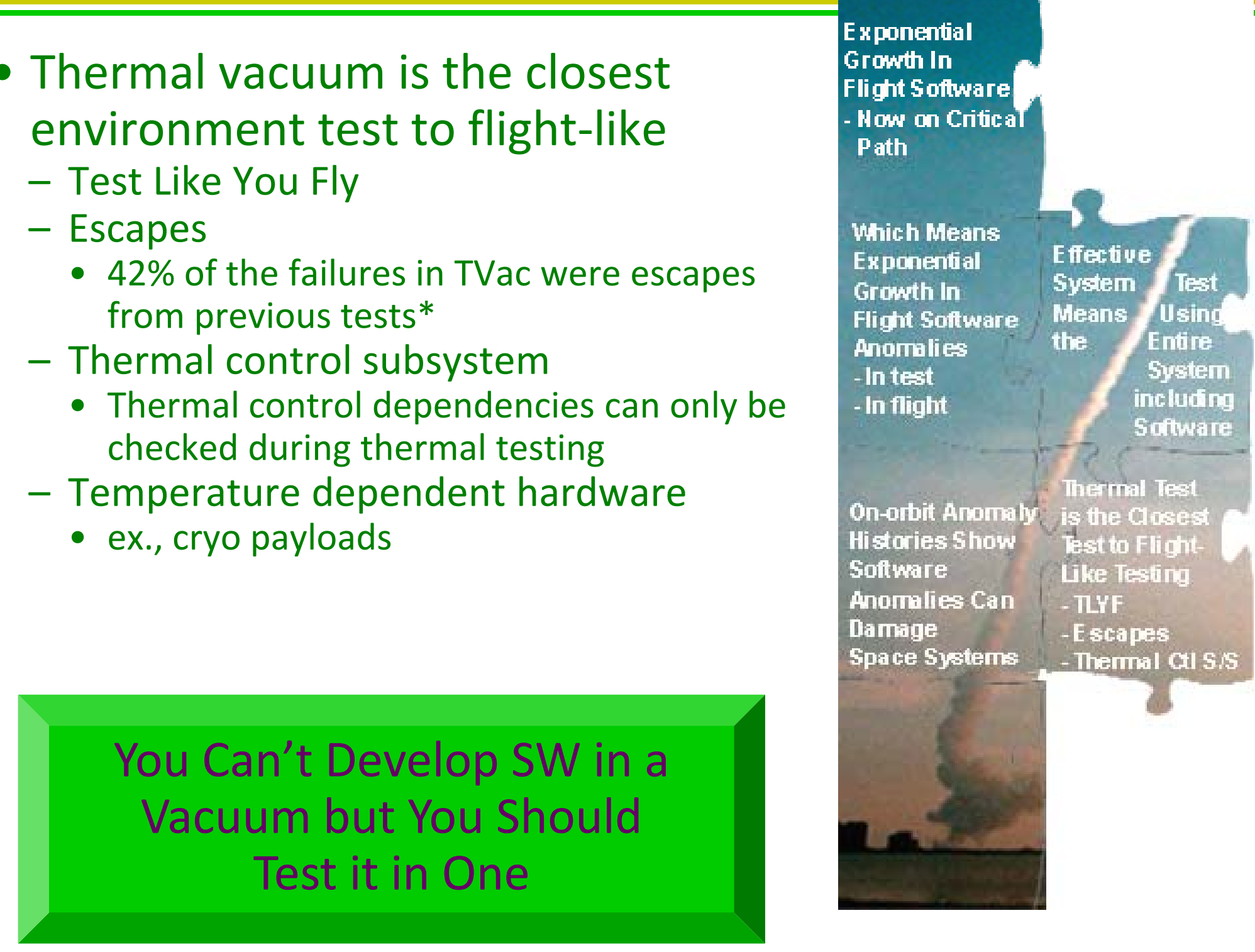


\section{Pieces of the Puzzle}

- Software related failures can occur at temperature

- Effective testing minimizes the anomalies encountered in flight

- Hardware/software interfaces

- Timing

- Timing can be thermally dependent

- Factory thermal anomaly data corroborates software failures

Final Flight Software Should be Used in TVac

Exponential

Growth In

Flight Software

- How on Critical

Path

Which Means

Exponential

Growth in

Flight Software

Anomalies

- In test

- In flight

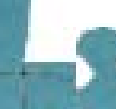

Effective

System

Means

the

Usin

Entire

System

including

Software

On-orbit Anomab

Histories Show

Software

Anomalies Can

Damage

Space Systems

Thermal Test

is the closest

lest to Fligh

Like Testing

- TLYF

- Escapes

- Themmal Cil Sis

Software Related

Failures Can

Occur at

Temperature

HWWSW Interfaces

- Timing 


\section{Pieces of the Puzzle}

- Anomaly data shows HW/SW interface issues are key

- Can no longer design and develop independently

- Research shows increased chance of flight failure with units being reworked after SV TVac

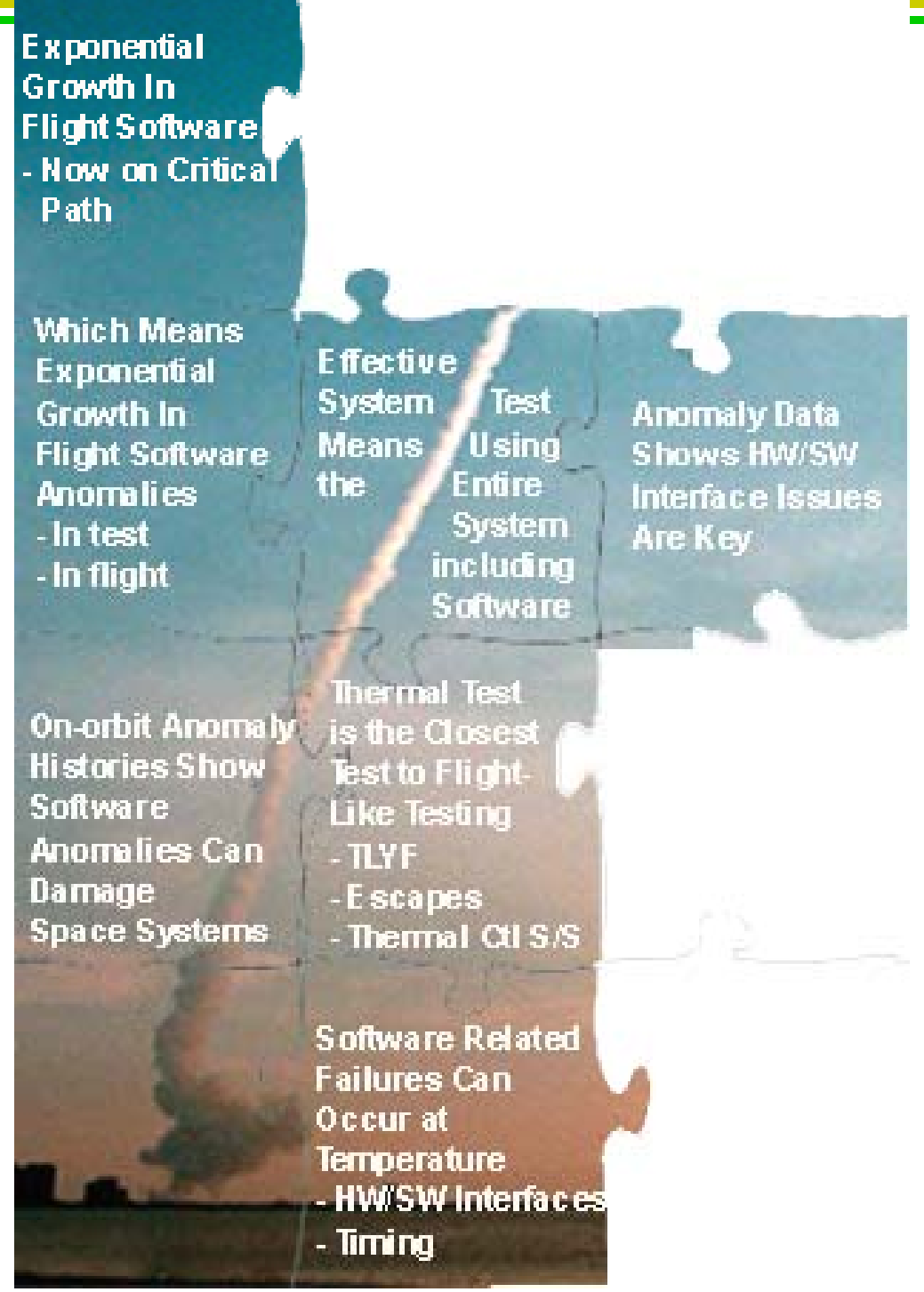




\section{Pieces of the Puzzle}

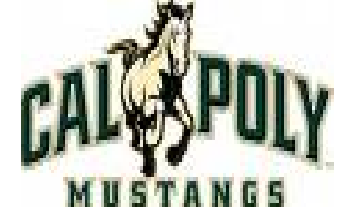

- More complex code

- More bugs

- Harder to find

- Takes longer to develop

- Increased chance that final software will not be ready by system test

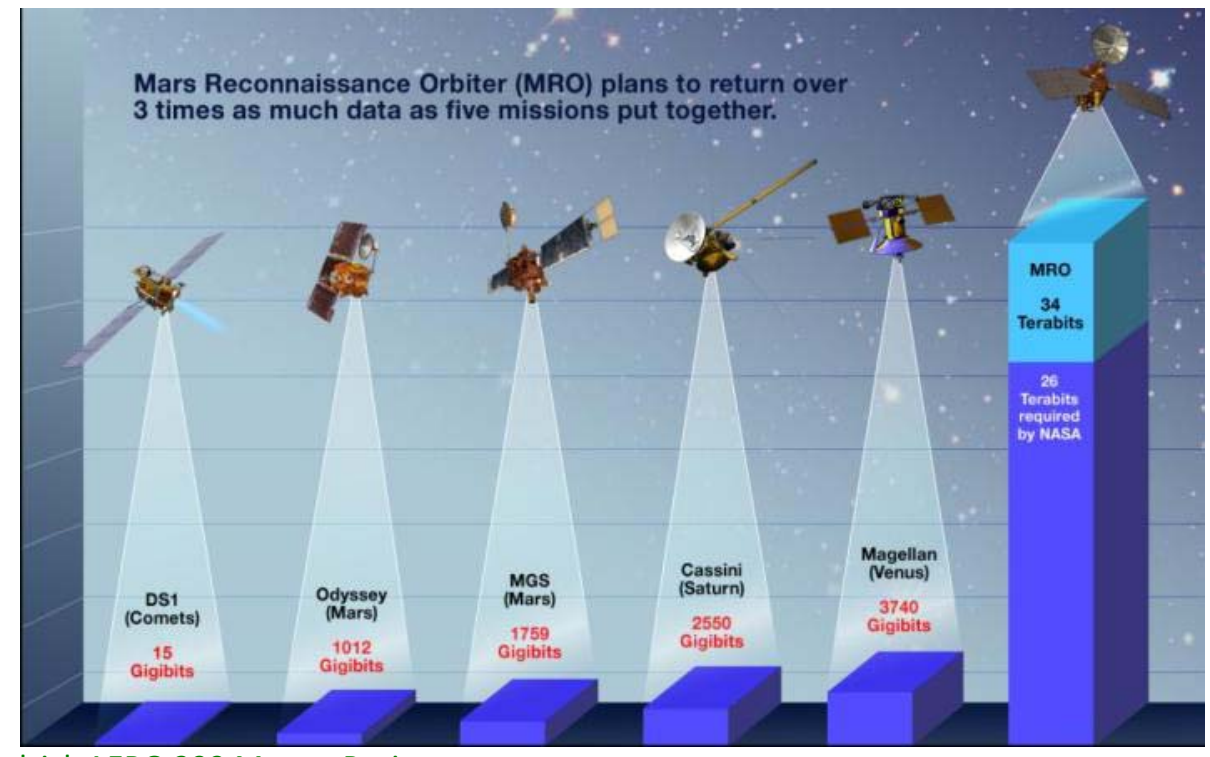

Exponential

Growth In

Flight Software

- How on Critical

Path

Which Means

Exponential

Growth In

Flight Software

Anomalies

- In test

- In flight

On-orbit Anomaby

Thermal Test

Histories Show is the Closest

Software

Anomalies Can

Damage

Space Systems

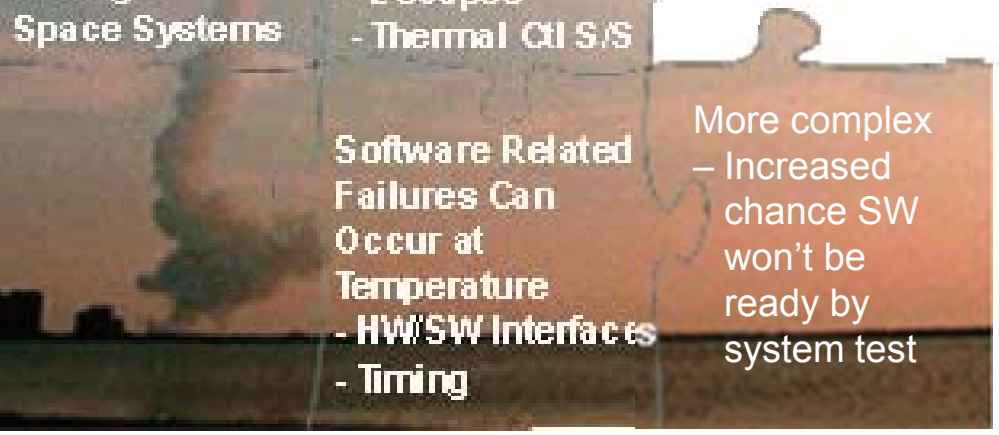




\section{Pieces of the Puzzle}

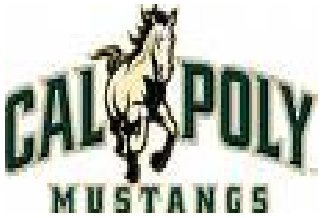

- Need to consider each case

- Programmatics

- Modularity

- Software complexity

- Mission criticality

- Software development process including thoroughness of checkout and verification

- Reuse vs modified

Exponential

Growth In

Flight Software

- How on Critical

Path

Which Means

Exponential

Growth In

Flight Software

Anomalies

- In test

- In flight

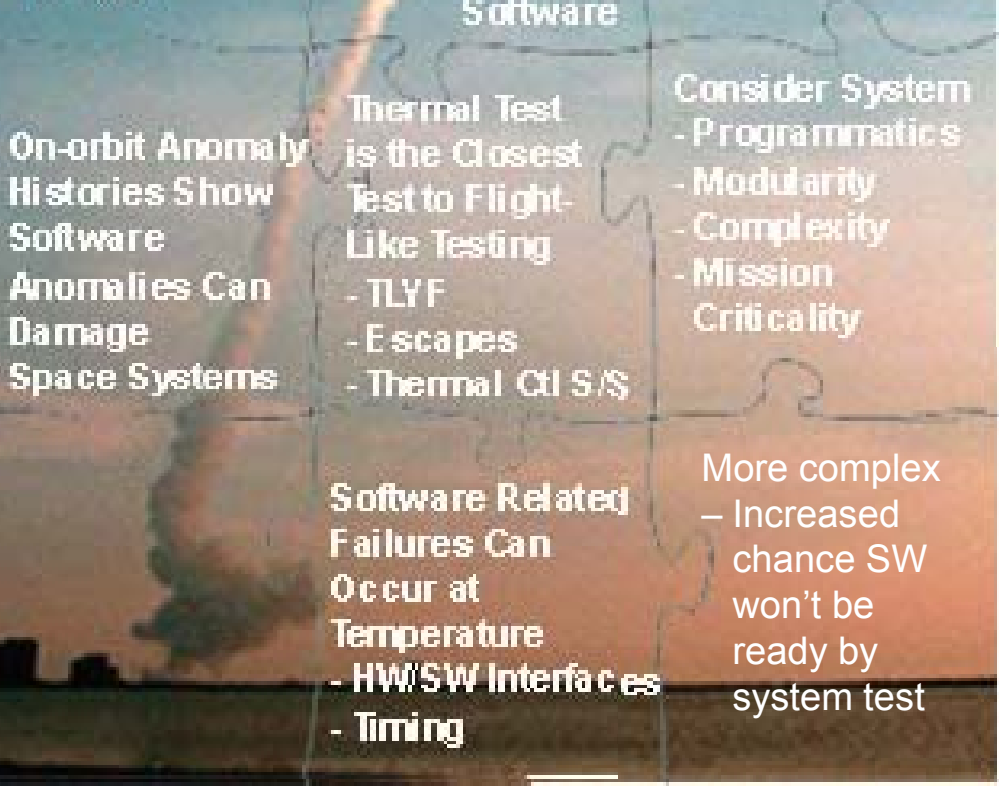

Software is playing a much more critical role in our mission success $-$

\section{Effective}

System

Means

the

IJsing

Entire

System

including

Software

Anomaly Data

Shows Hw/Sw

Interface lssues

Are Key

Consider System

Programmatics

Modtlarity

Complexits

Crilicality

Space Systems

occur at

HWS interace

system test 


\section{Pieces of the Puzzle}

- Effective testing minimizes the anomalies encountered in

flight

- Set up the test program and test at the correct levels

- Use the entire flight system (especially software)

- Understand, validate, and control the simulators and test beds

\section{The Choice is Ours}

\section{Exponential}

Growth In

Flight Software

- How on Critical

Path

Which Means

Exponential

Growth in

Flight Software

Anomalies

- In test

- In flight

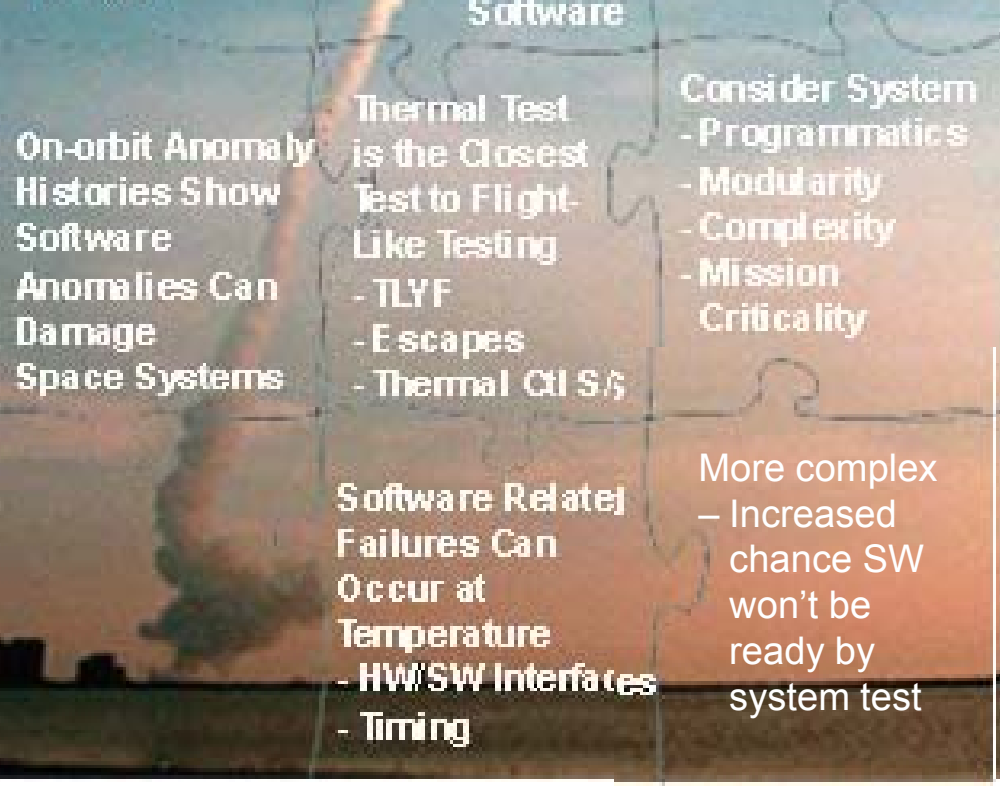

Effective

System

Means

Using

Entire

System

including

Anomaly Data

Shows HW/SW

Interface Issues

Are Key

Consider System

Programmatics

Missio

Anomalies Can

Damage

Space Systems 


\section{Pieces of the Puzzle}

\section{Our decisions create our destiny}

\begin{tabular}{|c|c|c|}
\hline $\begin{array}{l}\text { Exponential } \\
\text { Growth In } \\
\text { Flight Software } \\
\text { - Now on Critical } \\
\text { Path }\end{array}$ & & \\
\hline $\begin{array}{l}\text { Which Means } \\
\text { Exponential } \\
\text { Growth In } \\
\text { Flight Sottware } \\
\text { Anomalies } \\
\text { - In test } \\
\text { - In flight. }\end{array}$ & $\begin{array}{l}\text { Effective st } \\
\text { Means U, ing the } \\
\text { FULL S tem } \\
\text { inolud' ig } \\
\text { Soit are }\end{array}$ & $\begin{array}{l}\text { Anomaly Data } \\
\text { Shows hwisw } \\
\text { Interface Issues } \\
\text { Are Key }\end{array}$ \\
\hline $\begin{array}{l}\text { Increased Test } \\
\text { Anomalies } \\
\text { Impact Mission } \\
\text { Risk and } \\
\text { Sehedule. }\end{array}$ & $\begin{array}{l}\text { Thermal Test } \\
\text { is the closest } \\
\text { Test to Flight- } \\
\text { Like Testing } \\
\text { - TLYF } \\
\text { - Escapes } \\
\text { - Thermal Ct S/S }\end{array}$ & $\begin{array}{l}\text { Considar System } \\
\text {-Programmaties } \\
\text { - Modtarity } \\
\text { - Complexiby } \\
\text { - Mission } \\
\text { Criticality }\end{array}$ \\
\hline $\begin{array}{l}\text { Flight Failures } \\
\text { Show Software } \\
\text { Can Fail in } \\
\text { Flight } \\
\text { Resulting in }\end{array}$ & $\begin{array}{l}\text { Software Related } \\
\text { Failures Can } \\
\text { Deour at } \\
\text { Temperature }\end{array}$ & $\begin{array}{l}\text { More complex } \\
\text { - Increased } \\
\text { chance SW } \\
\text { won't be } \\
\text { ready by }\end{array}$ \\
\hline $\begin{array}{l}\text { Mission Failure } \\
\text { Degradation }\end{array}$ & $\begin{array}{l}\text { - HWW W Interfaces } \\
\text { - Ti ming }\end{array}$ & system test \\
\hline
\end{tabular}




\section{ANOMALIES}




\section{Stuff Happens}

- All hardware has flaws

- All software has bugs

- All interfaces (hardware to hardware, hardware to software, and software to software) have flaws beyond the individual elements

- Standard interfaces aren't

- Plan for anomalies - plan contingencies

- Design analysis frequently assumes ideal conditions

- Real life isn't ideal

- We may all speak English, but we don't speak the same language

\section{Murphy's Law Applies}




\section{Results Are Different than Expected}

\section{You have an anomaly!}

STOP) $S$ top what you are doing

W rite down the anomaly

A ssess the situation

涟 $T$ roubleshoot methodically to determine root cause

- Do NOT break configuration without consulting a wider audience

- Understand consequences of activities

- Get a wider look at the problem, potential causes, and consequences

Prevent the problem from occurring again

\section{Stop, Think, THEN Do}




\section{Anomaly Flow}
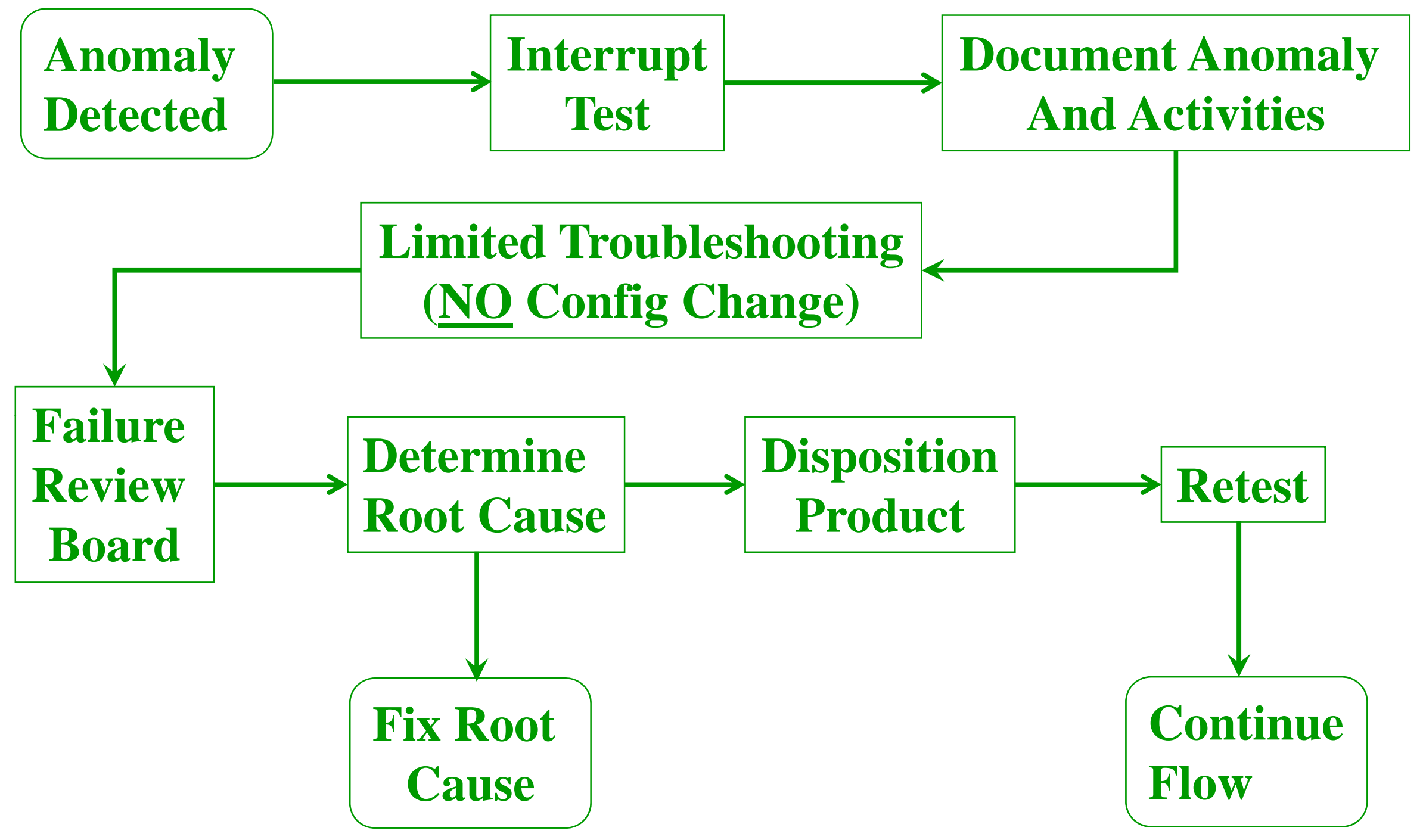


\section{Anomaly Indicators in Data}

- What the data tells you

- Out-of-limits

- States

- Trends

- Oddities (out of family)

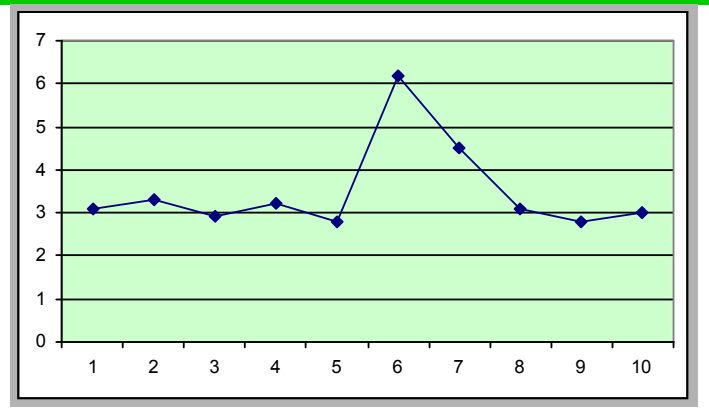

- The data won't tell you what you need to know if it is...

- Missed

- Data gathered but not analyzed

- Neglected

- Out of family but within specification

- "It only happened once"

- Explained away

- It must be a power glitch or noise; nothng in the flight product can explain it (but neither can the test product)

- Most of the observables explain it
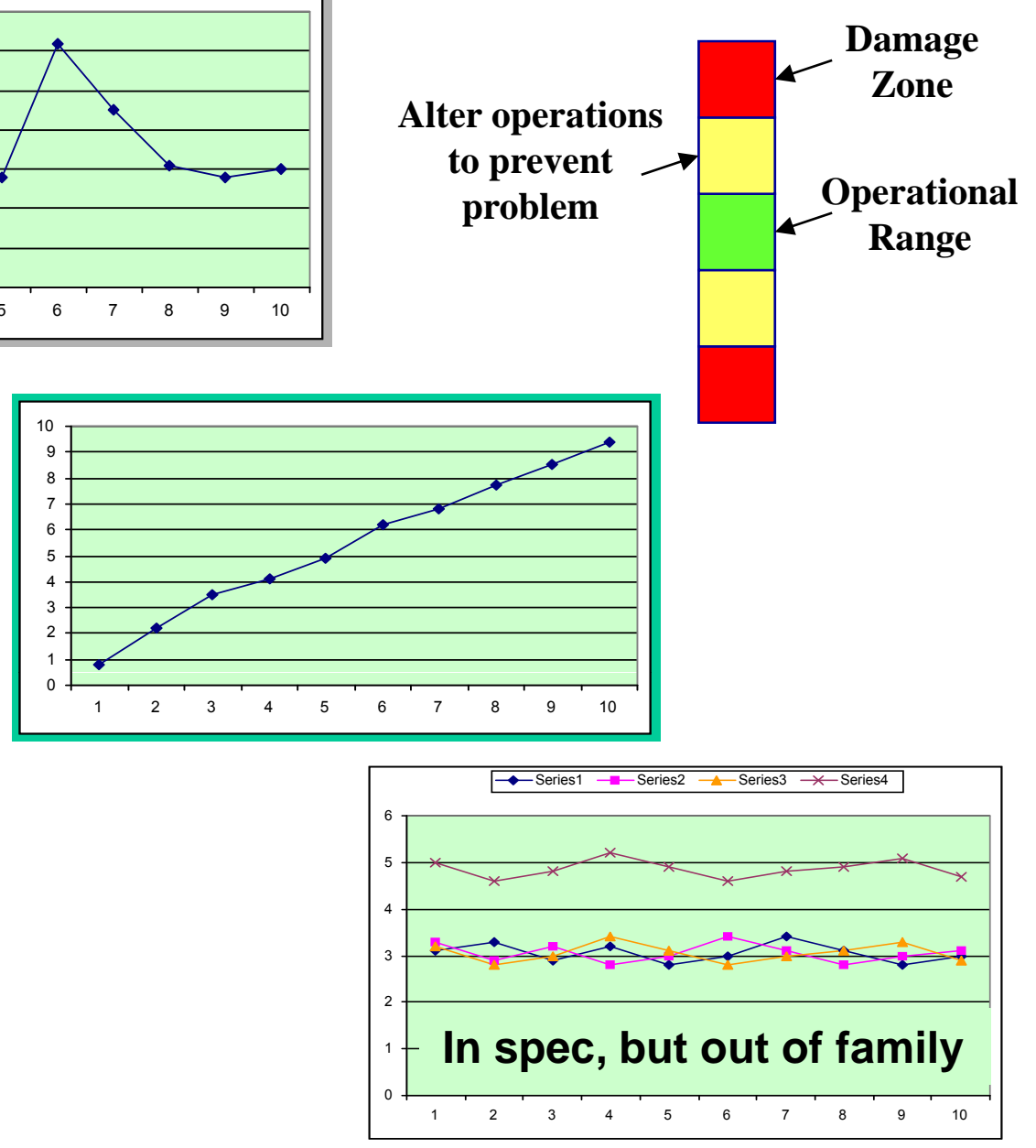

\section{Out of Family Data Needs to be Understood}




\section{Anomaly Tracking Issues}

- Data and anomalies need to be tracked across the life cycle

- Vendor

- Factory

- Launch site

- On-orbit
"The consequence of incomplete data capture systems can lead to sub-optimal or erroneous policy decisions, resulting in lower reliability and higher production costs."*

- Ownership of the process is an issue

- Many jurisdictions and systems/tools

- Quality, Test, Software Engineering, Hardware Engineering, Manufacturing, Reliability, Operations, etc

- No commonality and little overlap

- Fully closed loop process is essential

\section{Document It - Can't Address if Not Known}

*Kelly McGee and Alex Rubin, Failure Data Capture Tool: The Quest for an Open Standard, 20th Aerospace Testing Seminar 


\section{Five Common Mistakes}

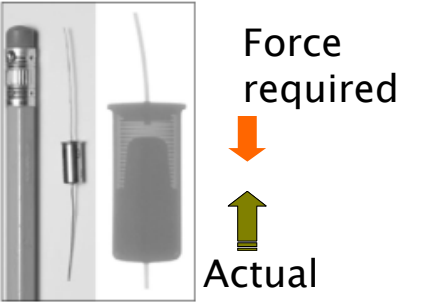

- Every vehicle has late changes - often difficult to verify

- Non-flight item removal, database update, harness mating, bracket

Reprinted courtesy of NASA installation, etc.

- Inability to cope with a computer hang-up

- Ensure the system can gracefully handle software glitches

- Misbehaving circuit protection devices

- Fuses, circuit breakers, and similar devices are hardware counterparts of fault management algorithm

- Often inadvertently "designed to blow"
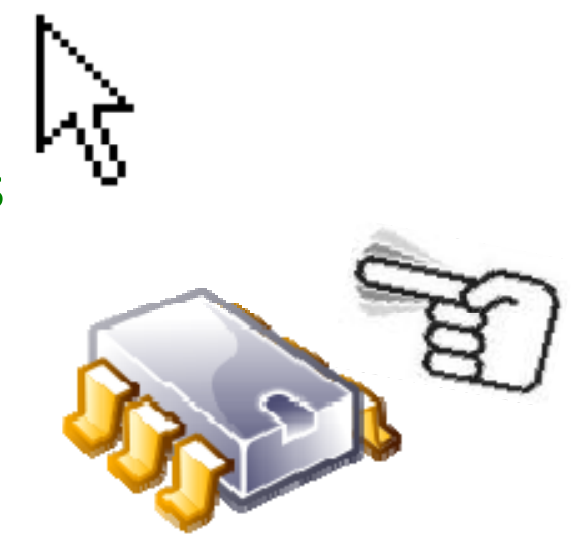

- Pyro safety deficiency

- Pyros impart large and irreversible shocks to the system

- Flying debris, post-firing shorts and structural shocks can cause damage

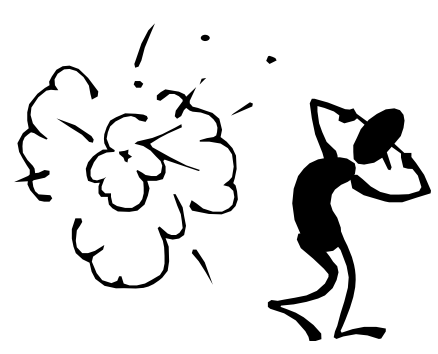




\section{What is Root Cause}

- Root cause is

- The actual or true agent(s) that cause a failure, anomaly, problem, or concern

- The underlying cause that led to the occurrence of the failure mechanism

- Preventing failures from reoccurring means discovering ALL of the root causes, not just the first cause you can identify

- Root Cause is known when

- All of the observed symptoms can be explained based on the physical description of the system

- No symptoms remain unexplained

- If root cause is not known, there is associated risk

\section{It May Not be A Single Root Cause}




\section{Root Cause Analysis}

- Root cause analysis consists of

- Investigation (includes troubleshooting)

- Analysis

- Decision (includes preventative actions)

- If you don't get to the root cause you may fix the immediate problem, but not the rest of what needs fixing

- Root cause will give you clues on where you may have other problems you haven't stumbled across yet

- We can spend a lot of time, effort, and money chasing solutions that merely mask the symptoms without diminishing the real problems

- Root cause is derived at the final or lowest level of analysis and is usually contained within the process, design, or workmanship parameters of the failed item

\section{Ask WHY Multiple Times}


Jefferson Memorial the Importance of Why
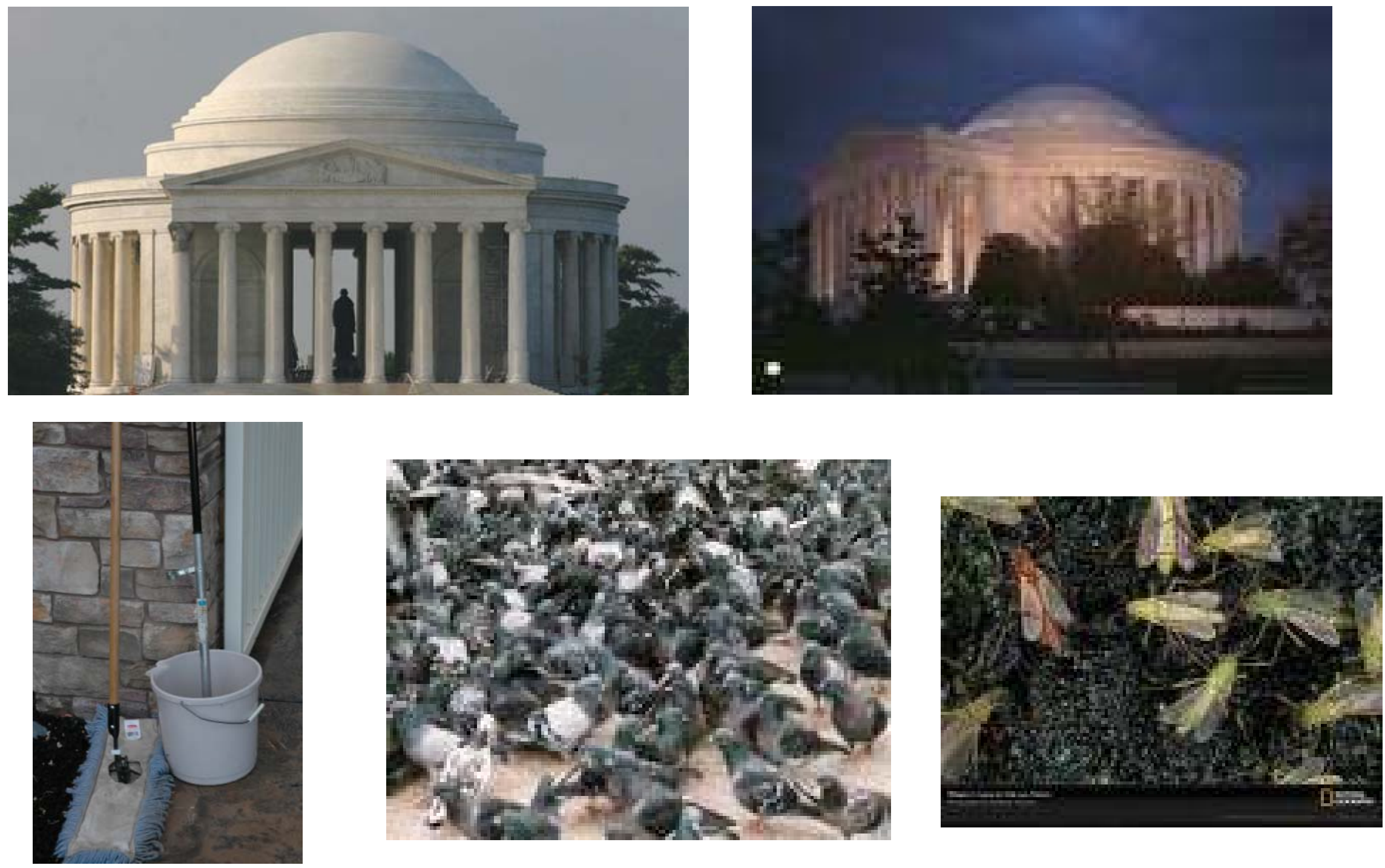


\section{Root Cause Analysis Tools}

- Decision Drawing / Logic Drawing

- Tree diagrams in which the selection of each branch requires some type of logical decision be made

- Causal Factor or Fault Tree / Event Tree

- Tree diagrams in which the selection of each branch requires the actions and conditions that were necessary and sufficient for a given consequence to have occurred

- Cause-and-Effect / Fishbone / Ishikawa diagrams

- Cause and effect diagram organized into a structure similar to that of a fishbone

Problem $\rightarrow$ Effect $\rightarrow$ "fish head"
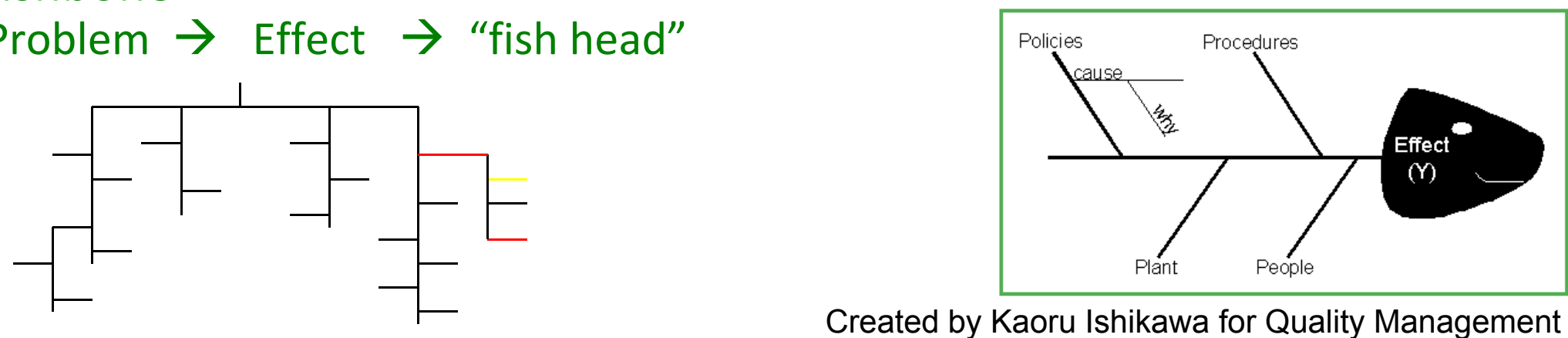

Created by Kaoru Ishikawa for Quality Management

\section{Use the Tools to Help Get Root Cause}




\section{Corrective Action}

- There are two levels of corrective action

- Disposition: Product Restoration

- Fix the product with the anomaly

- Preventative: Process Restoration

- Make sure the anomaly doesn't happen again

- Consider the "siblings"

- Look for and fix related products subject to the same root cause, even if they haven't "broken" yet

\section{Fix the Root Cause -}

Don't Just Keep the Product Moving 


\section{Retesting After Disposition}

- Retests must be performed to re-establish qualification, protoqualification and acceptance of reworked flight hardware, software, associated interfaces, and related interactions

- Rework and retest need the same system engineering persepctive and risk evalaution as original design, manufacturing, and test

- Decisions to be made

- How much performance and environmental retest is needed

- What was invalidated by troubleshooting and disposition

- What conditions are the retests

- Environments, cycles, etc

- What additional reviews and oversight are needed

- Understand the risks and impacts

\section{Verification is NOT Complete Until Test Passes}




\section{Retest Considerations}

- Hardware retest approach should be conservative

- Vibration before thermal at unit level

- Longer burn-in for high failure rate hardware

- Suggested minimum unit retesting

- Random vibration: three axes

- Thermal vacuum/thermal cycle: three cycles

- Repeat invalidated tests

- Repeat associated functional and/or performance tests

- Hardware changeout following System Test

- Penalty test plus normal retest

- Space vehicle retesting based on program risk assessment

- Extent and impact of R\&R assessed on case-by-case basis

- Assess residual risk of NOT retesting as part of decision process

- Unit pedigree traceability system provides valuable insight

- Track cumulative reworks / retests

- Calculate fatigue equivalence vs. margins

- Identifies potentially high risk hardware 


\section{Retest Considerations (cont)}

- Risks

- Not uncovering a 2nd (or 3rd) defect that was masked by the original

- Introducing new problems and secondary damage

- Cumulative Stress

- What defects will be missed if the retest is less robust than the original test in which the problem was detected?

- Out of sequence activities tend to be less rigorous than first build

- Heavy rework / late installation items increase risk of failure

- Heavy rework/retest

- 3X greater chance of infant mortality failure

- Late installation: After vehicle TVac

- Late installation often bypasses critical tests

- Heavy + Late causes increased flight anomalies

- Three times greater infant mortality rate

- Rework introduces less predictable failure modes 


\section{Conditions That May Drive Retest}

- Test anomalies

- Requirements change

- Change in flight environments

- Rework of previously tested hardware

- Change in materials or build processes 


\section{Retest Guidance from MIL-HDBK-340A}

- Amount of disassembly and reassembly

- "If hardware required considerable disassembly/reassembly, previous tests have likely been invalidated, even if repairs are relatively simple."

- Quantity and complexity of disconnects/reconnects

- "A repair requiring soldering or welding involves risk of damage to surrounding hardware which could invalidate previous tests."

- Access to inspect

- "If a repair can be inspected locally in the same manner as it was inspected during original manufacture, considerable confidence in its adequacy can be obtained."

" ... a repair which does not allow the same degree of inspection .. Has invalidated previous tests." 


\section{DEFECT DETECTION IN TEST}




\section{Defect Detection - Overview}

- The same defect can be found using more than one type of test/environment

- Leaky seals found in thermal vacuum, leak, and electromagnetic testing

- Shorted part found in performance, dynamic, thermal, and electromagnetic testing

- Chose verification method with correct perceptiveness at the correct level of integration

- Understanding the failure modes is key to downsizing

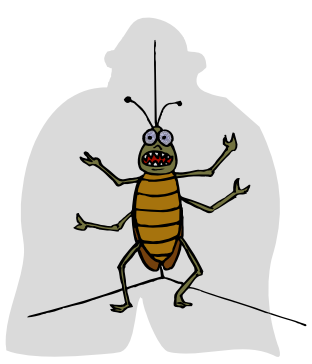

All Products have Defects

The Challenge is to Find the Critical Ones! 


\section{Defect Attributes/Trends}

\section{Design Mfg, Assy, I\&T Induced $\square$ Software Workmanship}

Most Frequent Attributes

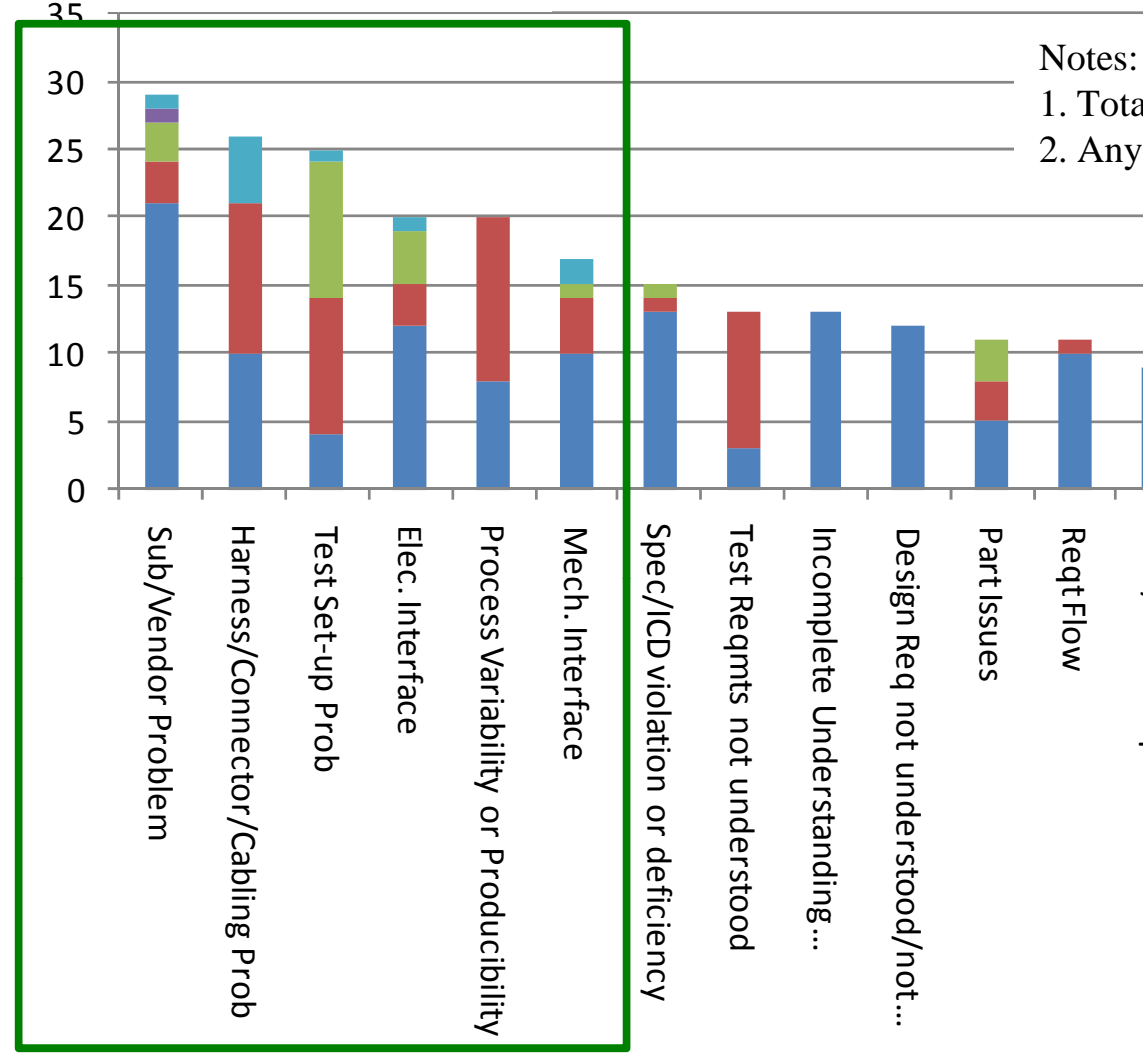

Total data set is 166 items.

Any given defect may have more than one attribute

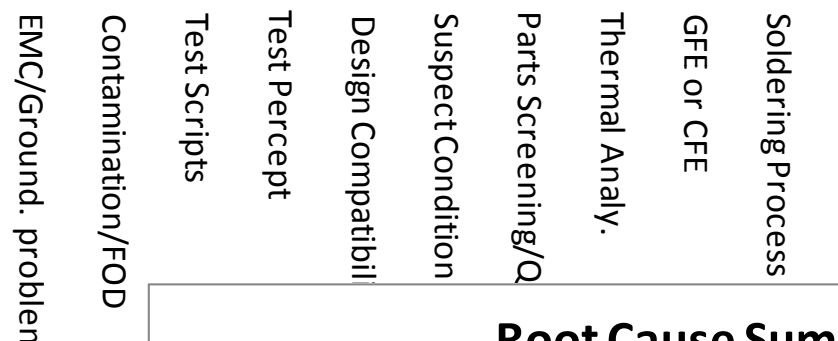

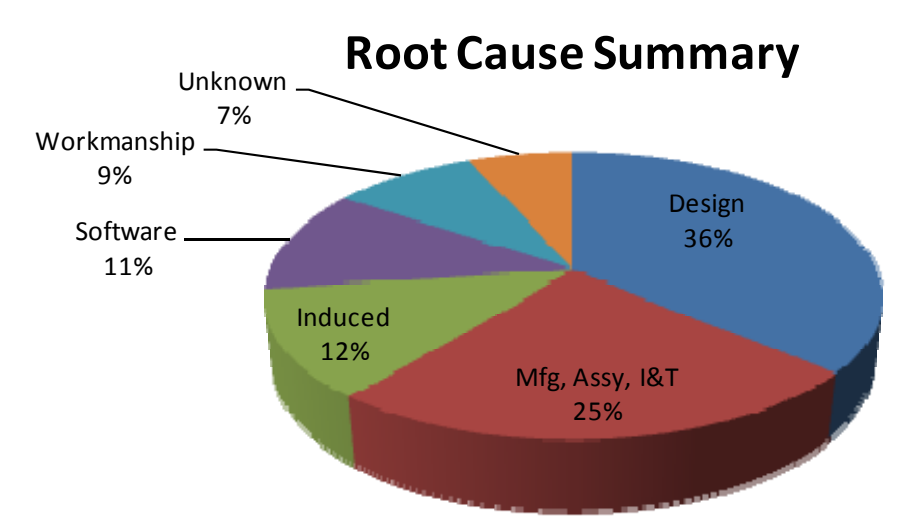




\section{Qualification vs Acceptance Failures}

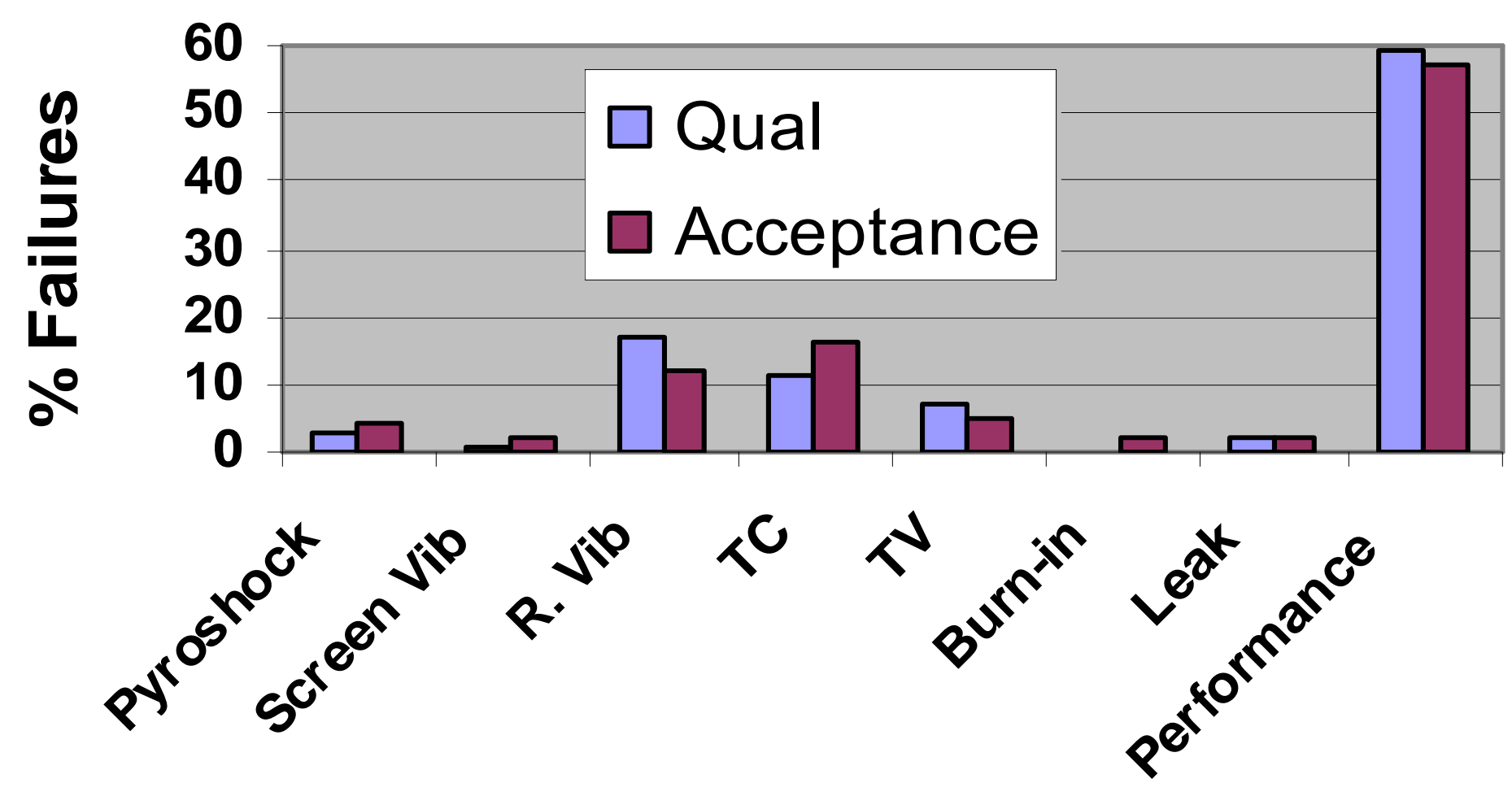

Test Environment

Performance Tests are Often the First Opportunity 


\section{HW Defects Identified by Qualification Tests}

\begin{tabular}{|c|c|c|c|c|c|c|c|c|c|c|}
\hline \multirow[b]{2}{*}{ Potential Failure Mechanism } & \multicolumn{10}{|c|}{$\begin{array}{c}\text { Primary Qualification Tests to Identify Failure } \\
\text { Mechanism }\end{array}$} \\
\hline & 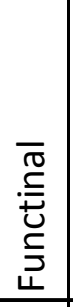 & 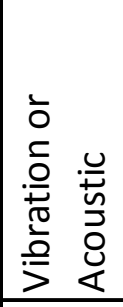 & \begin{tabular}{|l} 
\\
․ \\
o \\
v \\
\end{tabular} & 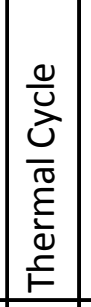 & 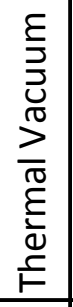 & 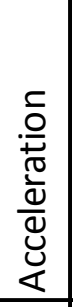 & 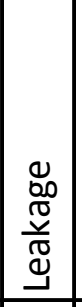 & 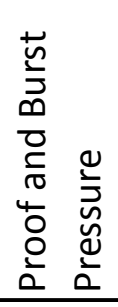 & $\sum_{\Psi}^{U}$ & 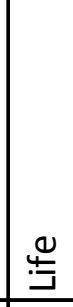 \\
\hline Mounting broken/loose & $\mathrm{x}$ & $x$ & $x$ & & & $x$ & & & $x$ & \\
\hline Broken part & & $\mathrm{x}$ & $\mathrm{x}$ & $x$ & $\mathrm{x}$ & & & & & \\
\hline Shorted part & $\mathrm{x}$ & $\mathrm{x}$ & & & $\mathrm{x}$ & & & & $\mathrm{x}$ & \\
\hline Defective part & $\mathrm{x}$ & $\mathrm{x}$ & & $\mathrm{x}$ & $\mathrm{x}$ & & & & $\mathrm{x}$ & \\
\hline Defective board & $\mathrm{x}$ & $\mathrm{x}$ & & $\mathrm{x}$ & $\mathrm{x}$ & & & & $\mathrm{x}$ & \\
\hline Broken/shorted/pinched wires & $\mathrm{x}$ & $\mathrm{x}$ & & $\mathrm{x}$ & $\mathrm{x}$ & & & & $\mathrm{x}$ & \\
\hline Defective/broken solder & $x$ & $x$ & & $x$ & $x$ & & & & $x$ & \\
\hline Contamination & & $\mathrm{x}$ & $\mathrm{x}$ & $\mathrm{x}$ & $\mathrm{x}$ & & & & & \\
\hline Leaky gaskets/seals/RF & & & & & $\mathrm{x}$ & & $\mathrm{x}$ & & $\mathrm{x}$ & \\
\hline Incorrect wiring/routing design & $\mathrm{x}$ & $\mathrm{x}$ & & & & & & & $\mathrm{x}$ & \\
\hline Relay/switch chatter & & $\mathrm{x}$ & $\mathrm{x}$ & & & & & & $\mathrm{x}$ & \\
\hline Adjacent circuit board contact & & $\mathrm{x}$ & $\mathrm{x}$ & & & & & & $\mathrm{x}$ & \\
\hline Premature wearout & & $\mathrm{x}$ & & & & & & & & $\mathrm{x}$ \\
\hline Electromagnetic interference & & & & & & & & & $x$ & \\
\hline Insufficient design margn & $\mathrm{x}$ & & & & & $\mathrm{x}$ & & $\mathrm{x}$ & $x$ & \\
\hline Corona discharge/arcing & & & & & $\mathrm{x}$ & & & & & \\
\hline Inadequeste tiedown of tubing/wiring & & $\mathrm{x}$ & & & & $\mathrm{x}$ & & & $\mathrm{x}$ & \\
\hline Inadequate thermal design & & & & $\mathrm{x}$ & $\mathrm{x}$ & & & & & \\
\hline Brittle material failure & & & $\mathrm{x}$ & & & & & & & \\
\hline Inadequate fatigue life & & $\mathrm{x}$ & & $\mathrm{x}$ & & & & & & $\mathrm{x}$ \\
\hline
\end{tabular}




\section{HW Defects Identified by Acceptance Tests}

\begin{tabular}{|c|c|c|c|c|c|c|c|c|c|c|}
\hline \multirow[b]{2}{*}{ Potential Failure Mechanism } & \multicolumn{10}{|c|}{ Primary Acceptance Tests to Precipitate Failure } \\
\hline & 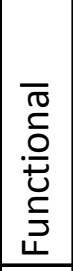 & 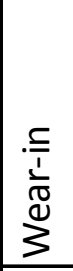 & 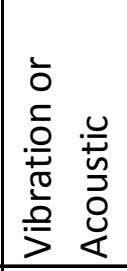 & $\begin{array}{l}\frac{\check{L}}{\Delta} \\
\dot{0} \\
\end{array}$ & 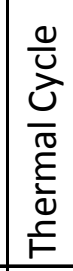 & 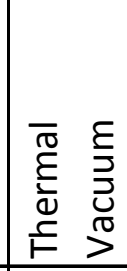 & 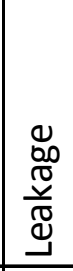 & 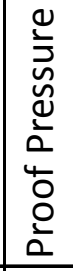 & \begin{tabular}{|l|} 
\\
0 \\
0 \\
0 \\
4 \\
0 \\
0 \\
0 \\
0 \\
\end{tabular} & 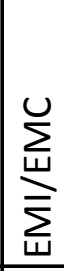 \\
\hline Parameter drift & $\mathrm{x}$ & & $x$ & & $x$ & $x$ & & & & \\
\hline $\begin{array}{l}\text { Electrical intermittants } \\
\text { - Solder joints } \\
\text { - Loose joints } \\
\text { - Connectors }\end{array}$ & & & $\mathrm{x}$ & $\mathrm{x}$ & $\mathrm{x}$ & $\mathrm{x}$ & & & & $\mathrm{x}$ \\
\hline Latent defective parts & $\mathrm{x}$ & & $\mathrm{x}$ & $\mathrm{x}$ & $\mathrm{x}$ & $\mathrm{x}$ & & & & \\
\hline Parts shorting & & & $\mathrm{x}$ & & & & & & & \\
\hline Chafed/pinched wires & & & $\mathrm{x}$ & & & & & & & $\mathrm{x}$ \\
\hline Adjacent circuit board contact & & & $\mathrm{x}$ & $\mathrm{x}$ & & & & & & \\
\hline Parameters changing due to deflections & & $\mathrm{x}$ & & & $\mathrm{x}$ & $\mathrm{x}$ & & & & $\mathrm{x}$ \\
\hline Loose hardware & & & $\mathrm{x}$ & $\mathrm{x}$ & & & & & & $\mathrm{x}$ \\
\hline Moving parts binding & & $\mathrm{x}$ & & & & $\mathrm{x}$ & & & & \\
\hline Leaky gaskets/seals & & & & & $\mathrm{x}$ & $\mathrm{x}$ & $\mathrm{x}$ & & & $\mathrm{x}$ \\
\hline Lubricants changing characteristics & & $\mathrm{x}$ & & & $\mathrm{x}$ & $\mathrm{x}$ & & & & \\
\hline Material embrittlement & & & & $\mathrm{x}$ & $\mathrm{x}$ & $\mathrm{x}$ & & & & \\
\hline Outgassing/contamination & & & $\mathrm{x}$ & $\mathrm{x}$ & & $x$ & & & & \\
\hline Degradation of electrical or thermal insulation & & & & & & $\mathrm{x}$ & & & & $\mathrm{x}$ \\
\hline Corona discharge/acrcing & & & & & & $\mathrm{x}$ & & & & $\mathrm{x}$ \\
\hline Defective pressure vessels & & & & & & & & $\mathrm{x}$ & & \\
\hline Structural defects & & & & & & & & & $\mathrm{x}$ & \\
\hline Defective wiring & $\mathrm{x}$ & & & & & & & & & $\mathrm{x}$ \\
\hline Defective tubing & & & & & & $\mathrm{x}$ & & & & \\
\hline
\end{tabular}




\section{SUMMARY}




\section{Summary}

- Verification (and validation) testing shall be traced to the requirements

- Understand difference between validation and verification

- Validation - Build the right thing

- Verification - Build the thing right"

- Pick the correct verification method for the requirement- D I A T

- Verification method decisions should be made with quality of proof in mind

- Verify and validate at the correct level - most cost and schedule efficient

- Ensure that the method addresses all key characteristics and an appropriate set of the variable space

- Where feasible, select test as the verification method of choice

- Determine pass/fail criteria BEFORE starting

- Understand fidelity for demonstration and analysis

\section{Validate, Verify, and Find and Fix Defects BEFORE Flight}




\section{Summary}

- Requirements verification process rolls it all up to provide proof that the final requirements have been met
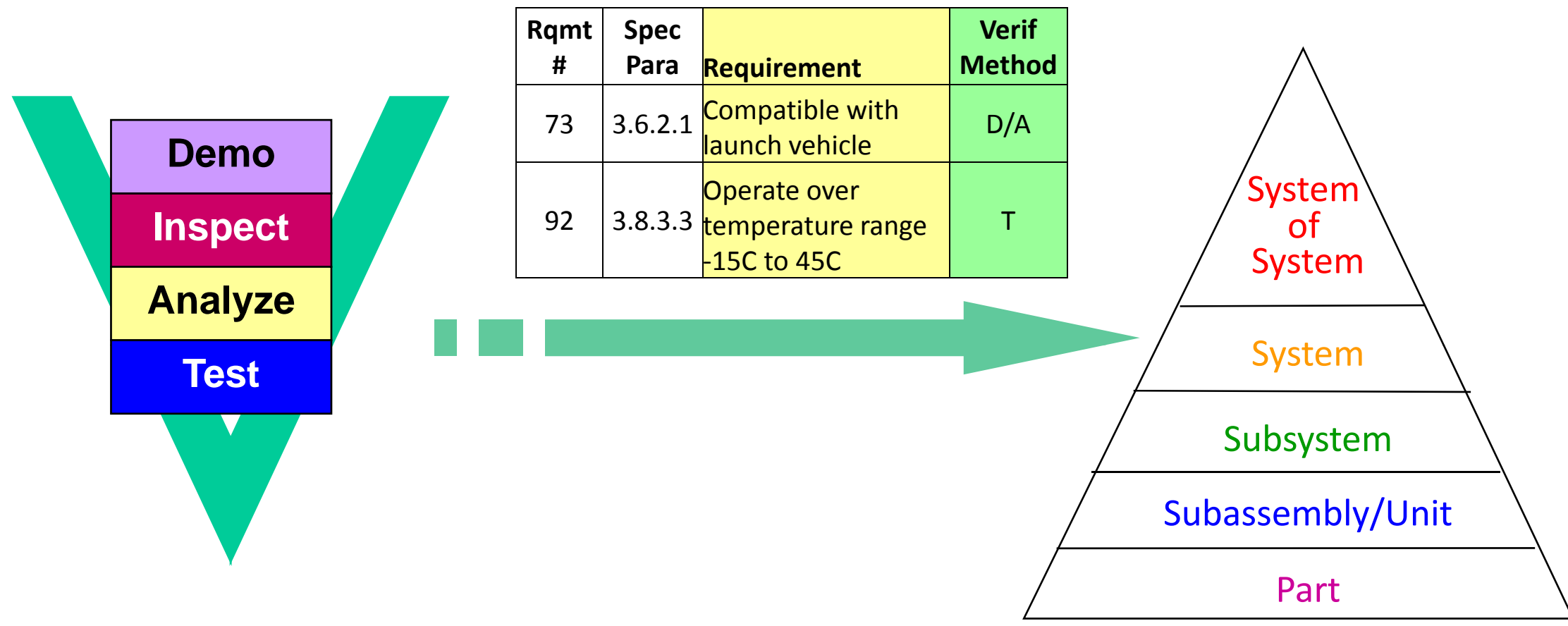

- Determine how to verify - requirement by requirement

- Find all the " $\mathrm{T}$ " and establish the test program 


\section{Summary}

- Test is a cumulative process

- Pyramid test philosophy

- Most effective test is at the appropriate level

- Establish and execute an effective test program early

- Keep the big picture in mind

- Understand the programmatics

- May require additional upfront commitment and new software development methods

- Identify hardware and software resources needed

- Choose the right test approach for your program

- All tests are valuable

- Removing tests is a function of Program risk tolerance

- It's always a balance of risk 


\section{Summary}

- There are a number of different environmental tests

- These tests are the foundation of a successful test program

- Understand and decide "why" and "what" test is before starting

- Test Like You Fly

- Software growth is posing a risk to the critical path

- Software is not a stand alone system

- Increase in failures and escapes both during system test and on-orbit

- Need to start qualification program early

- Execute planned and integrated software test program

- Peer reviews and product evaluation required prior to testing

- Use the software test process

- Understand limitations and differences of simulators and test beds

- Configuration management is essential 


\section{Summary}

- S W A T all anomalies

- Stop what you are doing

- Write down the anomaly

- Assess the situation

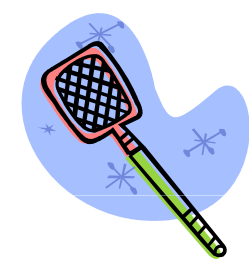

- Troubleshoot methodically to determine root cause

- Do NOT break configuration without consulting a wider audience

- Pursue anomalies to root cause

- Prevent the problem from occurring again

- Defects and errors are inherent in systems and manufacturing

- Proper problem identification, root cause determination and appropriate resolution are critical

- Rework and re-test need to be accomplished with the same perspectives and risk evaluation as the original design, manufacturing and test flow 
BACK-UP 


\section{Acronyms}

\section{CALl \\ MUSTAN G 5}

\begin{tabular}{|c|c|c|c|}
\hline$A B I$ & Advanced Baseline Imager & DSCS & Defense Satellite Communications System \\
\hline$A B L$ & Airborne Laser & DIAT & demonstration inspection analysis test \\
\hline ACS & Attitude Control Subsystem & DS1 & Deep Space 1 \\
\hline ACTS & Advanced Communications Technology Satellite & EMC & electromagnetic compatibility \\
\hline AFSCN & Air Force Satellite Control Network & EME & electromagnetic effects \\
\hline AGE & automated ground equipment & EMI & electromagnetic interference \\
\hline Al\&T & assembly, integration, and test & EPS & Electrical Power Subsystem \\
\hline \multirow[t]{2}{*}{ AIAA } & \multirow{2}{*}{$\begin{array}{l}\text { American Institute of Aeronautics and } \\
\text { Astronautics }\end{array}$} & ESA & European Space Agency \\
\hline & & ESD & electrostatic discharge \\
\hline AIM & Aeronomy of Ice in the Mesosphere & EUV & extreme ultraviolet \\
\hline aka & also known as & FAl & first article inspection \\
\hline ANSI & American National Standards Institute & FCM & flow control manifold \\
\hline ATE & automated test equipment & FF & full functional \\
\hline $\mathrm{C} \& \mathrm{DH}$ & command and data handling & FR & frequency response \\
\hline CE & conducted emission & \multirow[t]{2}{*}{ Gaia } & Global Astrometric Interferometer for \\
\hline CFE & radiated susceptibility & & Astrophysics \\
\hline CIV & critical ionization velocity & GFE & government furnished equipment \\
\hline \multirow[t]{2}{*}{ CNOFS } & \multirow{2}{*}{$\begin{array}{l}\text { Communication Navigation Outage Forecasting } \\
\text { System }\end{array}$} & GMI & GPM Microwave Imager \\
\hline & & GN\&C & guidance, navigation and control \\
\hline $\mathrm{CO} 2$ & carbon dioxide & \multirow[t]{2}{*}{ GOES } & \multirow{2}{*}{$\begin{array}{l}\text { Geostationary Operational Environmental } \\
\text { Satellite }\end{array}$} \\
\hline Comm & communication & & \\
\hline ConOps & concept of operations & GPM & Global Precipitation Measurement \\
\hline CONTOUR & Comet Nucleus Tour & GPS & Global Positioning System \\
\hline COTS & commercial off-the-shelf & GSE & ground support equipment \\
\hline CPU & central processing unit & HED & hall effect device \\
\hline CS & conducted susceptibility & HS & hot start \\
\hline CS & cold start & HST & Hubble Space Telescope \\
\hline
\end{tabular}




\section{Acronyms}

\begin{tabular}{|c|c|c|c|}
\hline HW & hardware & $\mathrm{P} / \mathrm{L}, \mathrm{PL}$ & payload \\
\hline I\&T & integration and test & PIM & passive intermodulation \\
\hline ICD & interface control drawing & PLF & payload fairing \\
\hline IEEE & Institute of Electrical and Electronic Engineers & PN & part number \\
\hline IES & Illuminating Engineering Society & PWA & printed wiring assembly \\
\hline IEU & Interface Electronics Unit & QC & quality control \\
\hline IR & infrared & R Vib & random vibration \\
\hline IRU & inertial reference unit & $R \& R$ & remove and replace \\
\hline ISS & International Space Station & RCA & root cause analysis \\
\hline IUS & inertial upper stage & RCS & Reaction Control Subsystem \\
\hline IV\&V & independent verification and validation & RE & radiated emission \\
\hline KPP & key performance parameter & RF & radio frequency \\
\hline KSLOC & thousands software equivalent lines of code & RFI & radio frequency interference \\
\hline LCU & load control unit & RS & radiated susceptibility \\
\hline \multirow[t]{2}{*}{ LMMS } & Lockheed Martin Missiles and Space (aka & S & stabilize \\
\hline & Lockheed Martin Space Systems Company) & S/B & should be \\
\hline LV & launch vehicle & $S / C$ & spacecraft \\
\hline MEOP & maximum expected operating pressure & $s / \mathrm{s}$ & stabilize and soak \\
\hline MGS & Mars Global Surveyor & SIQT & software item qualification testing \\
\hline MIRI & Mid InfraRed Instrument & SN & serial number \\
\hline MMA & moving mechanical assembly & SPR & software problem report \\
\hline MPE & maximum predicted environment & SRM & solid rocket motor \\
\hline MPT & maximum predicted temperature & SRS & shock response system \\
\hline MRO & Mars Reconnaissance Orbiter & SV & space vehicle \\
\hline NASA & National Aeronautics and Space Administration & SW & software \\
\hline $\mathrm{NiH}_{2}$ & Nickel Hydrogen & $T \& C$ & telemetry and command \\
\hline
\end{tabular}




\section{Acronyms}

\begin{tabular}{|l|l|}
\hline TAR & test anomaly report \\
\hline TC & thermal cycle \\
\hline TCS & Thermal Control Subsystem \\
\hline TERRIERS & $\begin{array}{l}\text { Tomographic Experiment using Radioactive } \\
\text { Recombinative lonosphere EUV and radio } \\
\text { Sources }\end{array}$ \\
\hline Thermal Ctrl S/S Thermal Control Subsystem \\
\hline TLYF & test like you fly \\
\hline TP & test procedure \\
\hline TT\&C & telemetry, tracking, and control \\
\hline TV & thermal vacuum \\
\hline TVac & thermal vacuum \\
\hline TWINS & $\begin{array}{l}\text { Two Wide-Angle Imaging Neutral-Atom } \\
\text { Spectrometers }\end{array}$ \\
\hline TWTA & traveling wave tube amplifier \\
\hline UHFFO & Ultra High-Frequency Follow-On \\
\hline UUT & unit under test \\
\hline Vib & vibration \\
\hline Xe & Xenon \\
\hline
\end{tabular}




\section{Bibliography}

Adams, R.J. et al. Software Development Standard for Space Systems. Aerospace Report No. TOR-2004(3909)-3537 Rev B.

El Segundo: The Aerospace Corporation, 2005.

Aguilar, Joseph A. Design Assurance Guide. Aerospace Report No. TOR-2009(8591)-11. El Segundo: The Aerospace

Corporation, 2009

Arnheim, B. and C. Belsick. "The Keys to Space Vehicle Integration and Test." 27th Aerospace Testing Seminar , 15 Oct 2012.

Arnheim, B. and D. Buettner. "The Need for Advanced Space Software Development Technologies." 23rd Aerospace Testing

Seminar. Oct 2006

Arnheim, B. and D. Buettner. "Putting Together the Pieces of Using the Final Flight Software During System Thermal Vacuum Testing."

3rd International Workshop of Verification and Testing of Space Systems . April 2009.

Arnheim, B.L. and P.S. Cheng. "The Price of Failure - A Study of System Test Anomaly Trends." El Segundo: The Aerospace

Corporation, 2005.

Asthana, Abhaya, and Jack Olivieri. "Quantifying Software Reliability and Readiness." 2009.

Beck, J. "GPS III Unverified Failures (UVFs) Process and Avoidance." Lockheed Martin, 2011.

Beizer, Boris. Software Testing Techniques . New York: Van Nostrand Reinhold, 1990

Bernhardt, Ken. "Driving to Root Cause and "the Rest of the Story"." CSE Community of Practice, 2009.

Boesiger, Ed. "Payload Wing Deployment Assembly Test Program." 27th Aerospace Testing Seminar. Oct 2012.

Buettner, Doug and Suellen Eslinger. "Software Testing Tutorial, Part 1: Software Testing Activities." 23rd Aerospace Testing

Seminar , 9 Oct 2006.

Cantrell, John, Eslinger, Suellen, and Robert Duvall. "A Program for Success: The Aerospace Role in Software Assurance."

Crosslink. Fall 2007: 26-31.

Cavano, Joseph P. "Quality Assurance in Future Development Environments." Software, IEEE . 4.5 (1987): 26 - 34

Cheng, Paul and Patrick Smith. 'Learning from Other People’s Mistakes." Crosslink. Fall 2007: 20-25.

Cheng, Paul and Tom Hecht. "Why Satellites Fail: Lessons for Mission Success." The Aerospace Corporation, 2009.

"Cover Blown." System Failure Case Studies 3.2 Feb 2009. NASA. Web. 9 Sept 2011. 


\section{Bibliography}

DOD-HDBK-343 Design, Construction, and Testing Requirements for One of a Kind Space Equipment. Washington, DC: USAF, 1986.

Durbin, Troy. "Focused Training Module Cause and Effect." Rev A. Lockheed Martin, 2007.

Eleazer, Wayne. "Launch failures: the "Oops!" factor." The Space Review. 31 Jan 2011.

Englehart, W.C., ed. Space Vehicle Systems Engineering Handbook . Aerospace Report No. TOR-2006(8506)-4494. El Segundo:

The Aerospace Corporation, 2005.

"Environmental Test Verification." The Midshipman Space Technology Applications Research (MidSTAR) Program. United States Naval Academy, 2007. Web. 9 Sep 2011. <http//www.usna.edu/Satellite/midstar/index.php?sect=tstplns>.

EPI 140-01 System Failure Resolution Best Practice . Lockheed Martin Corporation, 1997.

"Fender Bender." System Failure Case Studies 2.7 Sep 2008. NASA. Web. 9 Sept 2011.

"Genesis." Mishap Investigation Board Report. Vol I. NASA, Nov 2005.

Getting it Right . 1.1 The Aerospace Corporation. August 2010.

Getting it Right . 1.4 The Aerospace Corporation. Feb 2011.

Grattet, Vaun and Dave Shelton. "Affordable Mission Success". 27th Aerospace Testing Seminar . Oct 2012.

Guarro, S.B. and W.F. Tosney, ed. Mission Assurance Guide . Aerospace Report No. TOR-2007(8546)-6018, Rev A. El Segundo: The Aerospace Corporation, 2007.

Guarro, Sergio. "The Mission Assurance Guide: System Validation and Verification to

Achieve Mission Success." Crosslink. Fall 2007: 14-19.

Hanifen, D.W., A.J. Peterson, and W.F. Tosney, ed. Space Vehicle Test and Evaluation Handbook. Aerospace Report No. TOR2006(8546)-4591. El Segundo: The Aerospace Corporation, 2006.

Haskins, Cecelia, ed. Systems Engineering Handbook A Guide for System Life Cycle Processes and Activities . INCOSE-TP2003-002-03.2.2. San Diego: INCOSE, 2011.

Hecht, Myron and Douglas Buetnner. "Software Testing in Space Programs" Crosslink. Fall 2005: 31-35. 


\section{Bibliography}

Index of " System Failure Case Studies." NASA Safety Center. 9 Sept 2011. <http://nsc.nasa.gov/SFCS/Index/> Index of "United States Air Force Class A Aerospace Mishaps" Web. 9 Sept 2011. <http://usaf.aib.law.af.mil/> Index on various space mishaps and accidents. Massachusetts Institute of Technology, n.d. Web. 9 Sept 2011.

$<$ http://sunnyday.mit.edu/accidents/>

Johnson, Stephen B. "Success, Failure, and NASA Culture." NASA Ask Magazine. Fall 2008: 52-56.

Jolly, Steve. "Is Software Broken?" NASA Ask Magazine. Spring 2009: 22-25.

Jolly, Steve. "System of Systems in Space Exploration: Is Software Broken?" CSE Community of Practice, 2009.

Kabe, A.M., et al. 'Loads Analysis for National Security Space Missions”, Crosslink, Winter 2003: P 20-25.

Kowalchik, John (VP Mission Success, LMSSC). "Keynote Speaker." 27th Aerospace Testing Seminar 16 Oct 2012

Leveson, Nancy G. The Role of Software in Spacecraft Accidents. Massachusetts Institute of Technology, 2004.

$<$ http://sunnyday.mit.edu/papers/jsr.pdf $><$ http//sunnyday.mit.edu/>

Leveson, Nancy G., et al Effectively Addressing NASA’s Organizational and Safety Culture:

Insights from Systems Safety and Engineering Systems. Massachusetts Institute of Technology, 2004. < <ttp://sunnyday.mit.edu/>

Lions, J.L. "ARIANE 5 Flight 501 Failure." July 1996.

"List of spaceflight-related accidents and incidents." Wikipedia, n.d. Web. 9 Sep 2011.

$<$ http://en.wikipedia.org/wiki/List_of_spaceflight-related_accidents_and_incidents>.

Lloyd, James. "A Tale of Two Failures...the difference Between a "Bad Day" and a "Nightmare"." Leadership ViTS Meeting ,

NASA, 5 Dec 2005.

Lovarro, Doug (Executive Director AF Space and Missile System Center). "Keynote Speaker." 27th Aerospace Testing Seminar , 16 Oct 2012

Lue-Chung, Gary. "Apollo Root Cause Analysis (ARCA)." Structures to Problem Investigations . Lockheed Martin, 2011. Chapter 2.

Maier, Mark. "Architecting Principals for System-of-Systems." 6th INCOSE Symposium Proceedings, 1996 


\section{Bibliography}

McGee, Kelly and Alex Rubin. "Failure Data Capture Tool: The Quest for an Open Standard." 20th Aerospace Testing Seminar. May 2002 MIL-HDBK-340A Test Requirements for Launch, Upper-Stage, and Space Vehicles. Vol I: Baselines. El Segundo: Department of Defense, 1999.

MIL-HDBK-340A Test Requirements for Launch, Upper-Stage, and Space Vehicles. Vol II: Applications Guideline. El Segundo: Department of Defense, 1999.

MIL-STD-810G Test Method Standard Environmental Engineering Considerations and Laboratory Tests . Department of Defense , 2008.

MIL-STD-1522A, Standard General Requirements for Safe Design and Operation of Pressurized Missile and Space Systems . Washington DC:United States Air Force. 1984

MIL-STD-1547B, Electronic Parts, Materials, and Processes for Space and Launch Vehicles. Los Angeles: USAF Space and Missile Systems Center. 1992

MIL-STD-1833 Test Requirements for Ground Equipment and Associated Computer Software Supporting Space Vehicles. Los Angeles: United States Air Force, 1989.

"Mission Success Failure Analysis Tools." 2011.

Nagano, S. Space System Verification Program and Management Process. Aerospace Report No. TOR-2006(8506)-4732, Rev

A. El Segundo: The Aerospace Corporation, 2008

"New Tools for Testing Antennas." Crosslink . Winter 2002: 2.

Oberg, James. "Why the Mars Probe Went Off Course." SPECTRUM Magazine . Dec 1999.

O'Connor, Bryan and Jim Lloyd. "Cover Blown: WIRE Spacecraft Mishap." Leadership ViTS Meeting, NASA, Feb 2009.

O'Connor, Bryan and Jim Lloyd. "Fender Bender: DART's Automated Collision." Leadership ViTS Meeting, NASA, Sept 2008.

O'Connor, Bryan and Jim Lloyd. "STS-126: Shuttle Software Anomaly." Leadership ViTS Meeting, NASA, May 2009.

O'Connor, Bryan and Jim Lloyd. "The Million Mile Rescue: SOHO Lost in Space." Leadership ViTS Meeting, NASA, Nov 2008. 


\section{Bibliography}

"Overview of the DART Mishap Investigation Results." NASA. May 2006.

$<$ http://www.nasa.gov/pdf/148072main_DART_mishap_overview.pdf>

"Overview of the Orbiting Carbon Observatory (OCO) Mishap Investigation Results." NASA. July 2009.

$<$ http://www.nasa.gov/pdf/369037main_OCOexecutivesummary_71609.pdf $>$

Pennell, L.W. and B.E. Shaw, ed. System Engineering Requirements and Products. Aerospace Report No. TOR-2005(8583)-

3A. El Segundo: The Aerospace Corporation, 2005.

Penner, Andy. "SES Failure Review Board and Failure Analysis Training." 2011.

Perl, E., ed. Test Requirements for Launch, Upper-Stage, and Space Vehicles . Aerospace Report No. TR-2004(8583)-1A. El

Segundo: The Aerospace Corporation, 2006.

Perl, Erwin, Thinh Do, Alan Peterson, and John Welch. "Environmental Testing for Launch and Space Vehicles." Crosslink. Fall 2005: 11-15.

Quart, K.L. and I. Wong Proceedings IEEE Reliability and Maintainability Symposium, IEEE, New York, 1990

"Report on Project Management in NASA by the Mars Climate Orbiter Mishap Investigation Board." SpaceRef Interactive Inc.

NASA HQ. 13 March 2000

Rubin, Alex. "Anomaly Detection and Resolution (ADR) Technical Training Modules." Lockheed Martin, 2004 updated 2011.

Rubin, Alex. "Problem Investigation and Resolution A Structured Approach". 2011.

Sarafin, Thomas P., ed. Spacecraft Structures and Mechanisms From Concept to Launch . Microcosm, Inc., 1995.

"Shuttle Software Anomaly" Special Study: NASA Close Call NASA Safety Center, Apr 2009.

SMC Systems Engineering Primer and Handbook . 3rd. USAF Space and Missiles Systems Center: April 2005.

SMC-S-001 Systems Engineering Requirements and Products . El Segundo: Air Force Space Command Space and Missile Systems Center, 2010.

SMC-S-012 Software Development for Space Systems . El Segundo: Air Force Space Command Space and Missile Systems

Center, 2008. 


\section{Bibliography}

SMC-S-016 Test Requirements for Launch, Upper-Stage, and Space Vehicles. 1st. USAF Space and Missiles Systems Center: June 2008

Snyder, James. "Introduction to SMC-S-016 (Mil-Std-1540E) Test Requirements for Launch, Upper-stage \& Space Vehicles." 27th Aerospace Testing Seminar, 15 Oct 2012.

"SOS/SE Characteristics and Definition." Capability Integrated Architecture Development (CIAD) Working Group, 14 Dec 2006.

Space Primer A Student Guide to the Principles of Space. El Segundo: The Aerospace Corporation, 2003.

Strang, Norm. "Ground System Testing." Crosslink. Fall 2005: 36-38.

"Systems Engineering." INCOSE, August 1997.

Systems Engineering Handbook A "How To" Guide for All Engineers . Version 2.0. Seattle: INCOSE, 2000.

Systems Engineering Process. El Segundo: The Aerospace Corporation, 2003.

Tanner, C.S. "Satellite Acceptance Test - Updated Study of Acoustic Test Effectiveness." Spacecraft/Launch Vehicle Dynamics

Environments Workshop. The Aerospace Corporation, June 2001

Tanner, Tom. "A Game Loop Architecture for the Modeling and Simulation of Mission Threads." 13th Annual Systems Engineering

Conference, National Defense Industrial Association (NDIA), 25-28 Oct 2010.

"The Million Mile Rescue." System Failure Case Studies 2.9 Nov 2008. NASA. Web. 9 Sept 2011.

"The Tour Not Taken." System Failure Case Studies 3.7 Oct 2009. NASA. Web. 9 Sept 2011.

"Titan IV-32 Mishap Report" USAF Accident Investigation Board. <sunnyday.mit.edu/accidents/titan...rpt.doc >

"Titan IVA-20 Accident Investigation Board Summary." USAF Accident Investigation Board.

$<$ http://www.globalsecurity.org/space/library/report/1999/titan_iv-20_sum.htm>

Tosney, Bill and Steve Pavlica. "A Successful Strategy for Satellite Development and Testing." Crosslink. Fall 2005: 6-10.

Welch, John W. Flight Unit Qualification Guidelines. Aerospace Report No. TOR-2010(8591)-20. El Segundo: The Aerospace

Corporation, 2010

Wertz, James Richard, and Wiley J. Larson, ed. Space Mission Analysis And Design. 3rd. Microcosm Press, 1999. 


\section{Bibliography}

"What is System of Systems Engineering." National Centers for System of Systems Engineering. Old Dominion University, Norfolk, VA 23529, 13 Nov 2007. Web. <http:/www.eng.odu.edu/ncsose/What_is_SOSE.shtml>.

White, Julia. Test Like You Fly (TLYF): Assessment and Implementation Process. Aerospace Report No. TOR-2010(8591)-6. El Segundo: The Aerospace Corporation, 2010

Worth, Daniel B and Rodney N. Phillips. "Results of the HESSI Test Mishap Investigation." NASA. Dec 2002.

$<$ http://ntrs.nasa.gov/archive/nasa/casi.ntrs.nasa.gov/20010020956_2001027911.pdf> 